\title{
ANALYSIS OF TUMBLE RATE AND SPIN AXIS ORIENTATION FOR ROCKET BODIES NEAR GEOSTATIONARY ALTITUDE
}

\author{
A Thesis \\ presented to \\ the Faculty of California Polytechnic State University, \\ San Luis Obispo
}

\author{
In Partial Fulfillment \\ of the Requirements for the Degree \\ Master of Science in Aerospace Engineering
}

by

Kimberly Andersen

June 2014 
(C) 2014

Kimberly Andersen

ALL RIGHTS RESERVED 


\section{COMMITTEE MEMBERSHIP}

TITLE:

AUTHOR:

DATE SUBMITTED:

COMMITTEE CHAIR:

COMMITTEE MEMBER:

\author{
Dr. David Mitchell, Associate Professor,
} Department of Physics

COMMITTEE MEMBER:

Dr. John Keller, Associate Professor, Department of Physics

COMMITTEE MEMBER:

Department of Aerospace Engineering 


\begin{abstract}
Analysis of Tumble Rate and Spin Axis Orientation for Rocket Bodies near Geostationary Altitude
\end{abstract}

Kimberly Andersen

This report describes the results of optical observations of several Ariane 5 rocket bodies near geostationary altitude. Their estimated sidereal spin rates and spin axis orientations are obtained by a combination of analyzing the light curves of the rocket bodies and the 3D geometry between the observer and the sun. Knowing the sidereal tumble rate and the spin axis orientation will be helpful for future debris removal missions if the number of artificial satellites near Earth's geostationary orbit has any hope of decreasing. In addition, by generating light curves for these satellites, it will help future optical observations of unknown objects be classified as Ariane 5 rocket bodies if their light curve is similar to those presented in this paper (or not classify them as an Ariane 5 rocket body if the light curves are dissimilar). The data presented in this paper were taken throughout April and May of 2014 at the Cal Poly San Luis Obispo Astronomical Observatory. A Meade 14" telescope and CCD camera were used for data collection, and data was reduced using IRAF software and self-written Matlab code. 


\section{ACKNOWLEDGMENTS}

Special thanks to my committee chair Dr. Kira Abercromby for always being so positive and encouraging when I was very behind schedule or had done things incorrectly. Many thanks to Dr. David Mitchell for all the help with data reduction, always being available for questions, and for sanity checking my data analysis procedures. To John Keller, thanks for encouraging me not to give up hope when all was cloudy (literally, it was cloudy), and thanks to Dr. Jordi Puig-Suari for being there when I needed him. Without my committee, I would not be able to present this document!

In addition to thanking my committee, I would also like to thank the sponsors of the Baker Science Building which is where the funding for the brand new Meade 14" telescope came from. More than that, I want to express my deep gratitude to the observatory technician, Steve Soderberg, without whom the new telescope would not work at all! I don't know what I would have done without your willingness to continue working on the telescope-computer connection errors. 


\section{TABLE OF CONTENTS}

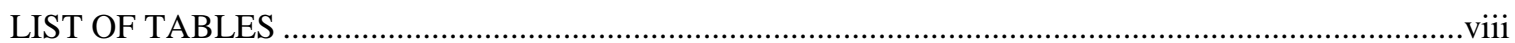

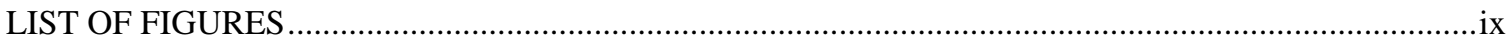

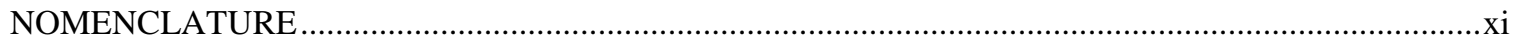

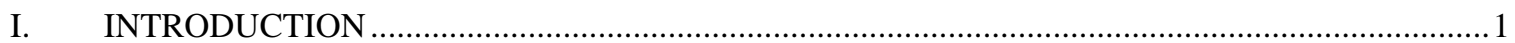

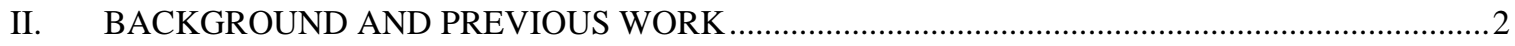

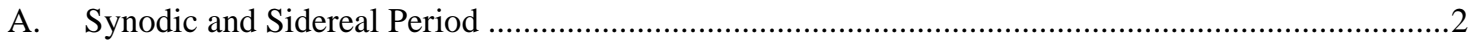

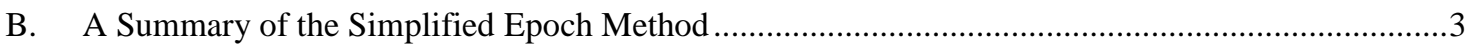

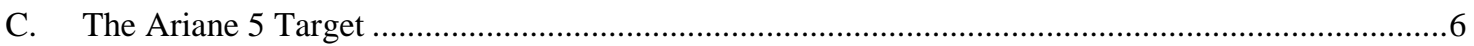

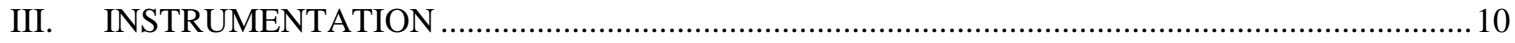

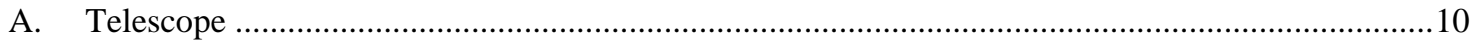

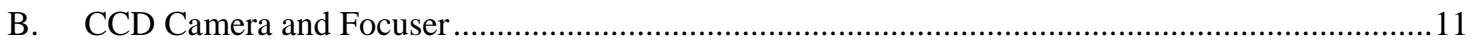

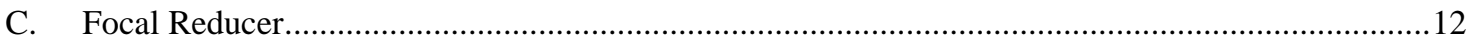

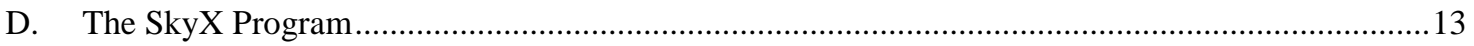

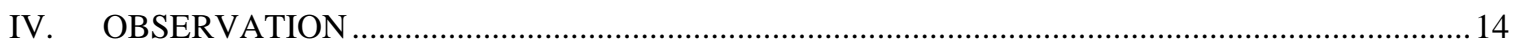

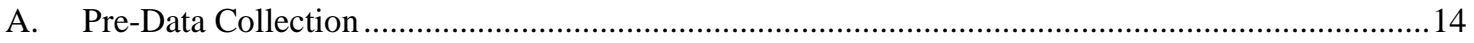

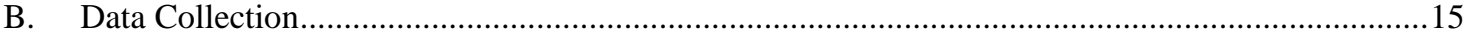

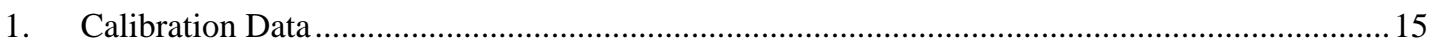

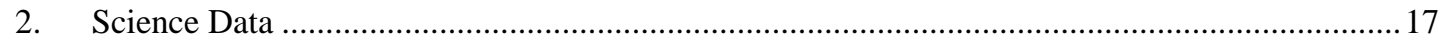

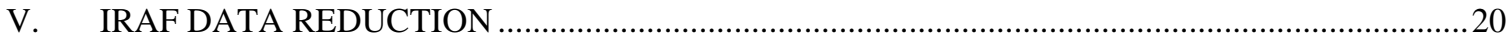

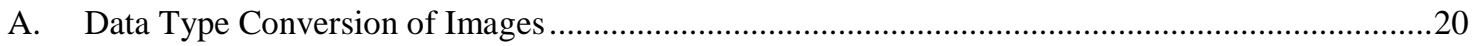

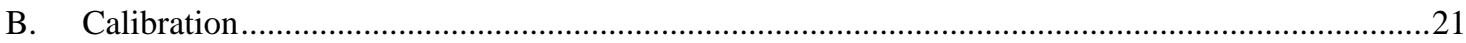

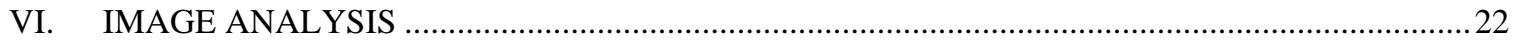

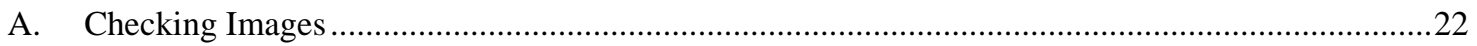

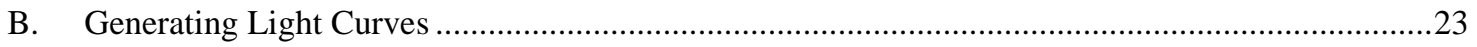

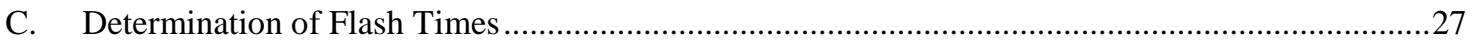

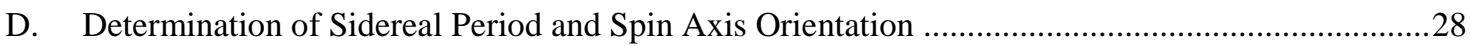




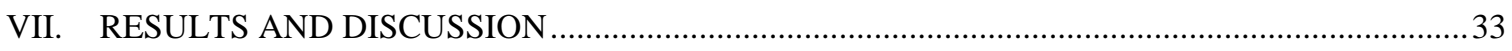

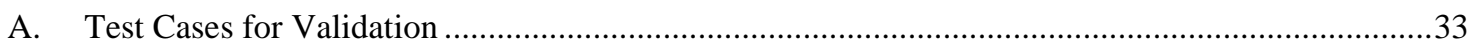

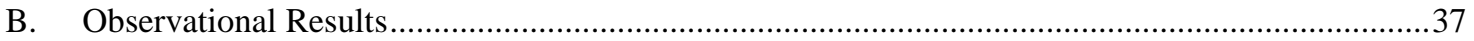

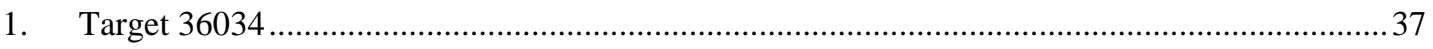

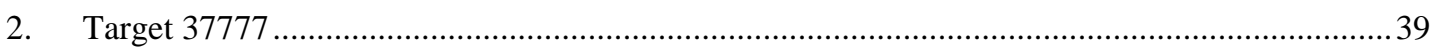

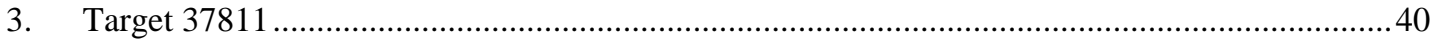

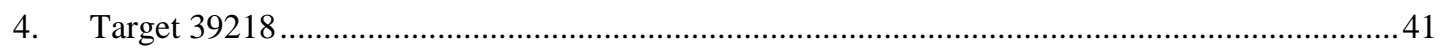

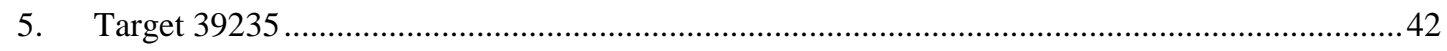

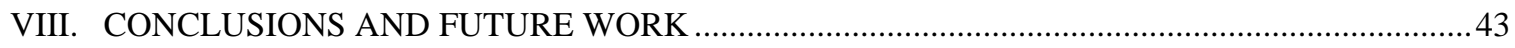

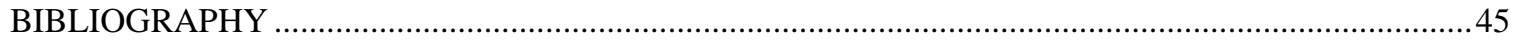

APPENDICES

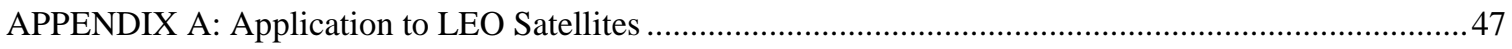

APPENDIX B: List of Components and Software Used......................................................................50

APPENDIX C: Cal Poly Observatory Telescope Set-Up and Shut-Down Procedures ..................................51

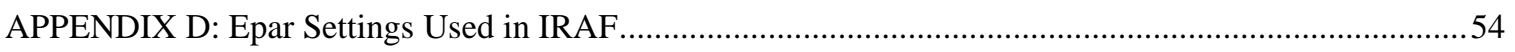

APPENDIX E: Selected Light Curves for Analyzed Data .....................................................................56

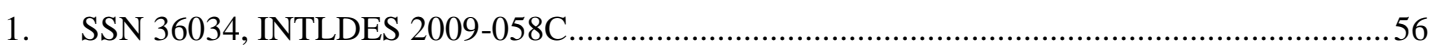

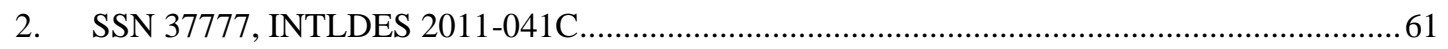

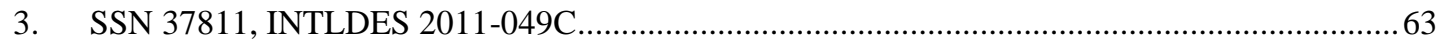

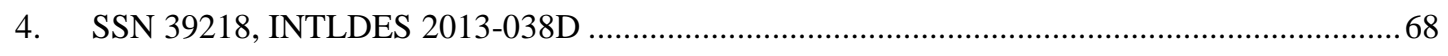

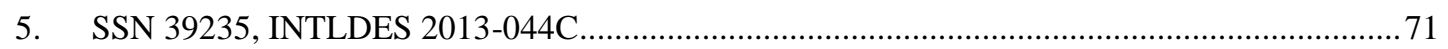

APPENDIX F: Contour Plot Results for Observational Data ……...........................................................73

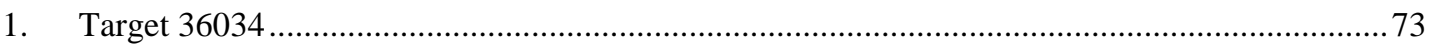

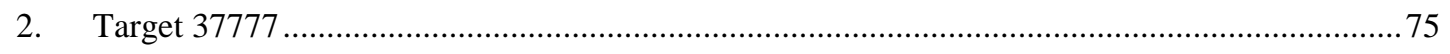

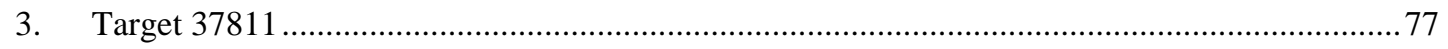

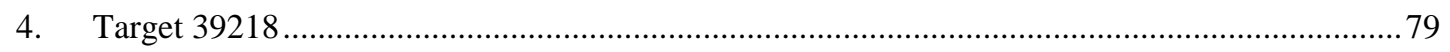

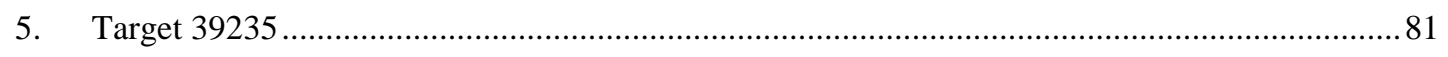




\section{LIST OF TABLES}

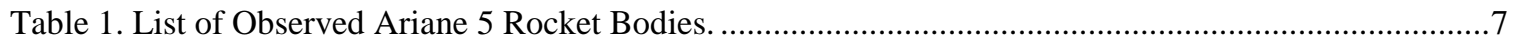

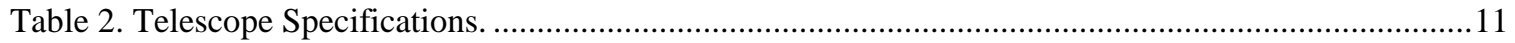

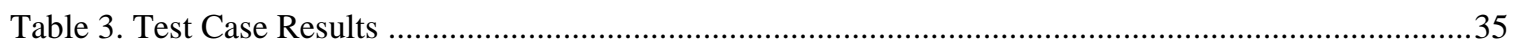

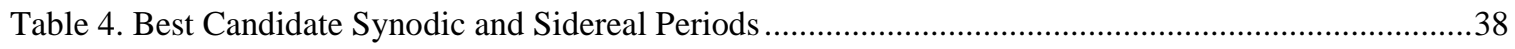

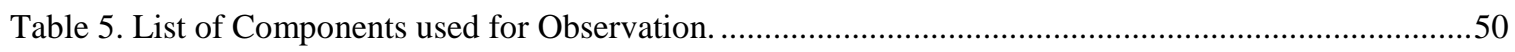

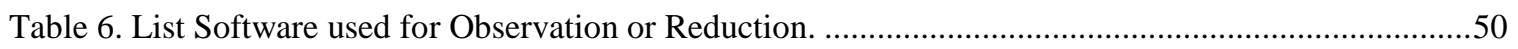

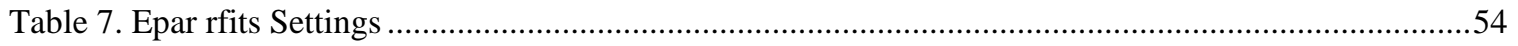

Table 8. Epar Zero, Epar Dark, and Epar Flat Settings ........................................................................54

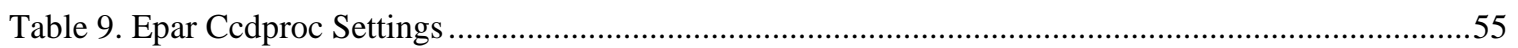




\section{LIST OF FIGURES}

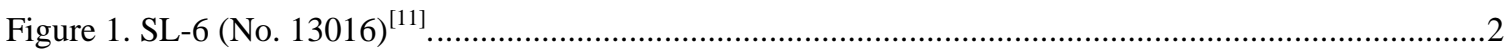

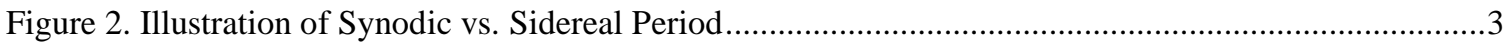

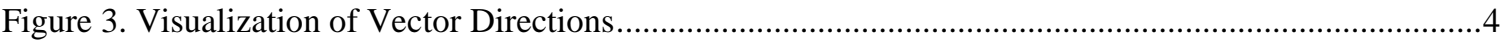

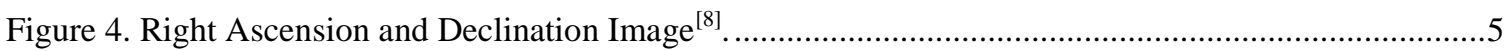

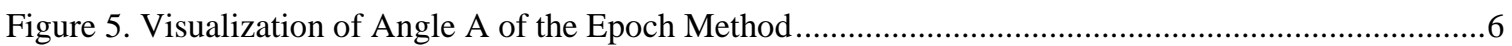

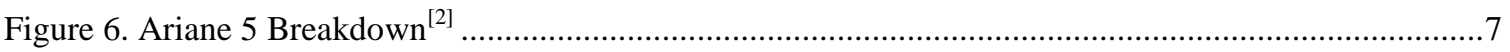

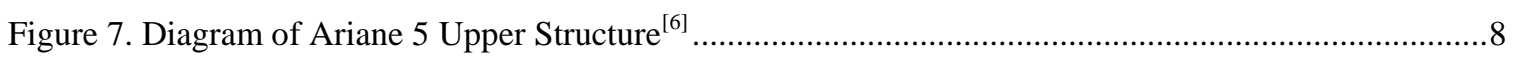

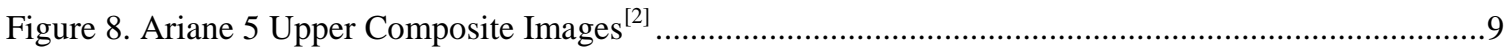

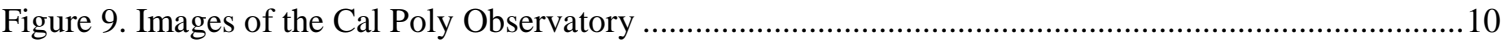

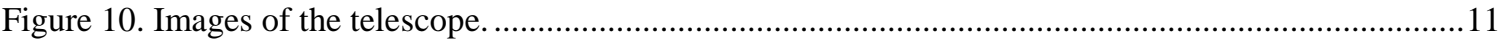

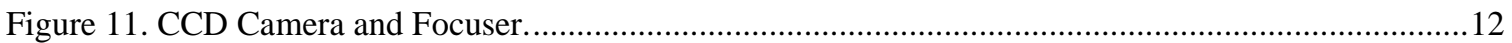

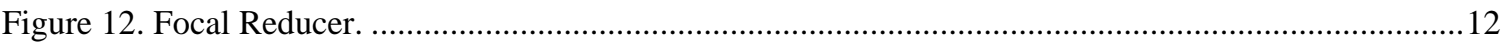

Figure 13. Flowchart for Data Collection..............................................................................................

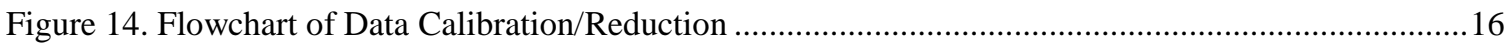

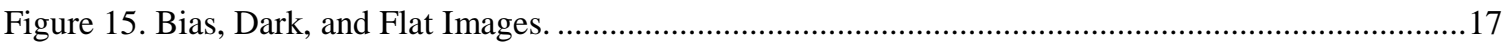

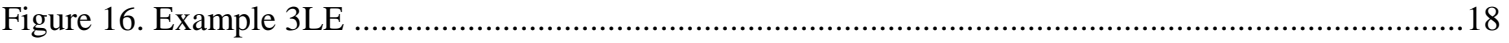

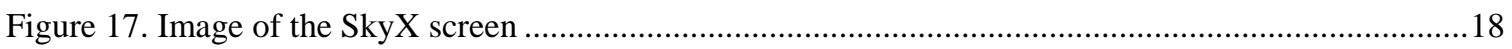

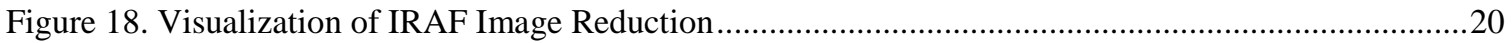

Figure 19. Visualization of Matlab used for Image Analysis ...................................................................22

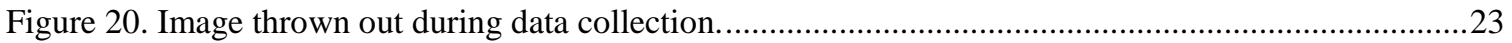

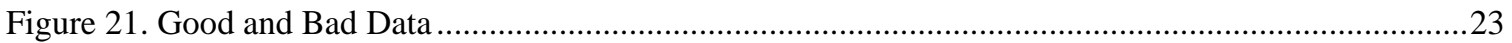

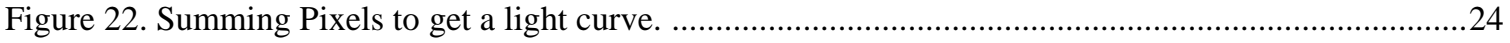

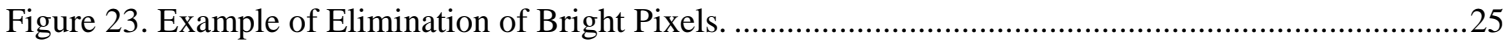

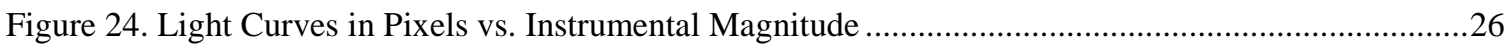

Figure 25. Example Light Curves for Obtaining Flash Times. …….............................................................2 


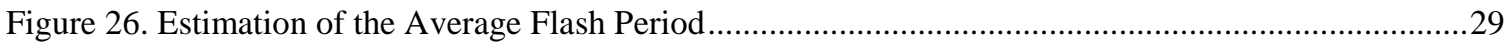

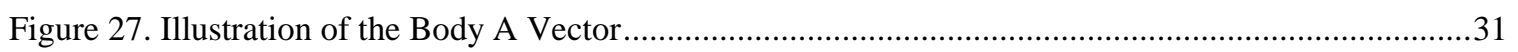

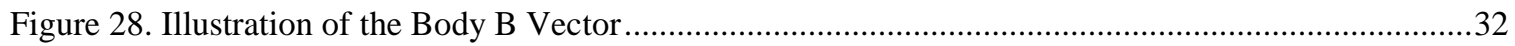

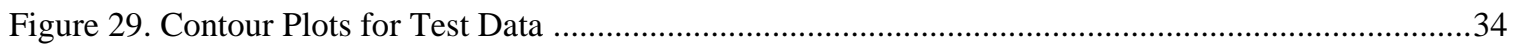

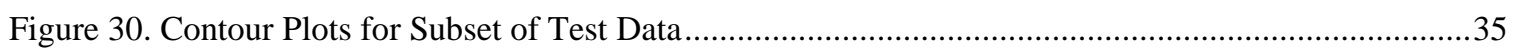

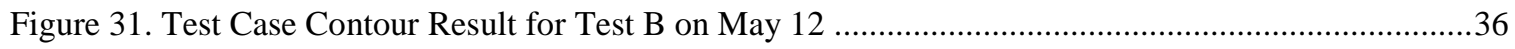

Figure 32. Sidereal Period Score vs. Sidereal Period ...............................................................................

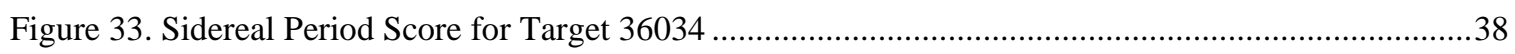

Figure 34. Sidereal Period Score for Target 37777 ..................................................................................39

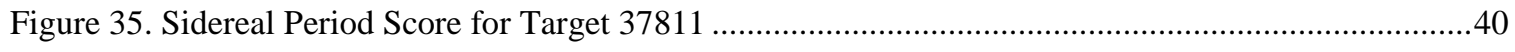

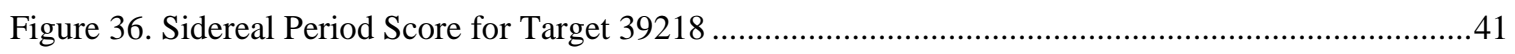

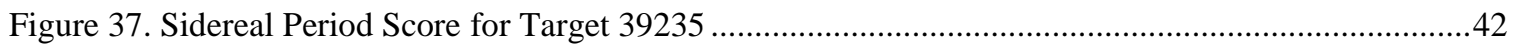

Figure 38. Contour Plot for First Pass of the LEO Test Case Satellite ........................................................48

Figure 39. Contour Plot for Second Pass of the LEO Test Case Satellite ...................................................48

Figure 40. Contour Plot for Both Passes of the LEO Test Case Satellite ..................................................49 


\section{NOMENCLATURE}

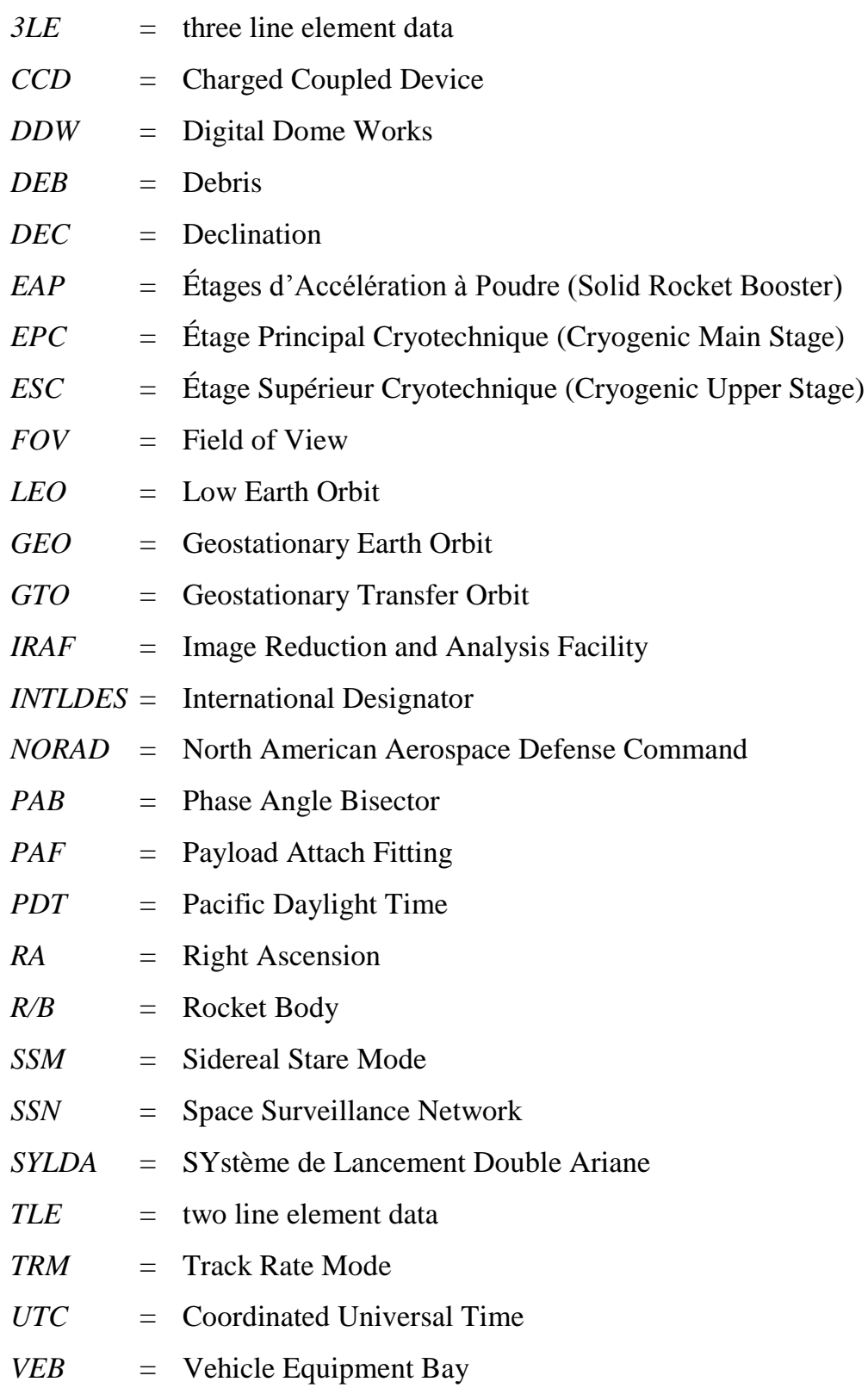




\section{INTRODUCTION}

Orbital debris is becoming a larger problem in space because there is no effective method for collecting debris, only regulations to control the number of debris objects. Even then, spacecraft that fail before their predicted end of life sometimes cannot follow these regulations for disposal as intended. The first step toward collecting debris would be to determine the orbit, attitude, and tumble or spin rate to prepare for a mission that could attempt to capture this debris and de-orbit it, or at least rendezvous with it and move it to a new orbit where it will not affect other active spacecraft ${ }^{[4]}$. To prove the point that space is becoming crowded: while collecting data for this research, at least five other unintentional objects (either operational satellites or other debris pieces) streaked through the FOV of the CCD camera, including one that followed the actual target through several images.

Space collisions are the reason this is becoming a more and more important issue. Each time a collision occurs, the number of space debris objects increase, which increases the likelihood of another collision. The number of space debris objects will therefore grow exponentially unless something can be done. Rogue pieces of space debris can damage or destroy operational satellites, so avoidance maneuvers are performed if debris is predicted to pass nearby which uses fuel and may affect normal satellite operations. Space is becoming more and more dangerous for operational satellites, as if the other problems of space (radiation, thermal, power, perturbations) are not enough to worry about when designing a spacecraft.

A geostationary orbit is a 24 hour period orbit that allows for continuous coverage over one ground location, therefore it is a very desirable orbit that leads to many operational satellites crowded together in the same area. If debris passes through the geostationary belt it could disrupt many satellites, making it particularly important for active debris removal in this area. Therefore, results presented in this paper are the tumble rates and spin axis orientations of several rocket bodies near geostationary altitude orbits. 


\section{BACKGROUND AND PREVIOUS WORK}

Several objects were observed by Wallace, Somers, and $\operatorname{Scott}^{[14]}$, and a few more observations were published later by Somers ${ }^{[11]}$. These combined results included the "candidate" spin axis and sidereal periods for 11 different objects, seven of which were SL-6 rocket bodies and the other four were decommissioned Boeing/Hughes satellites. Because little is known about the exact values, their published numbers are given in the hopes of being verified or refuted by future analysis.

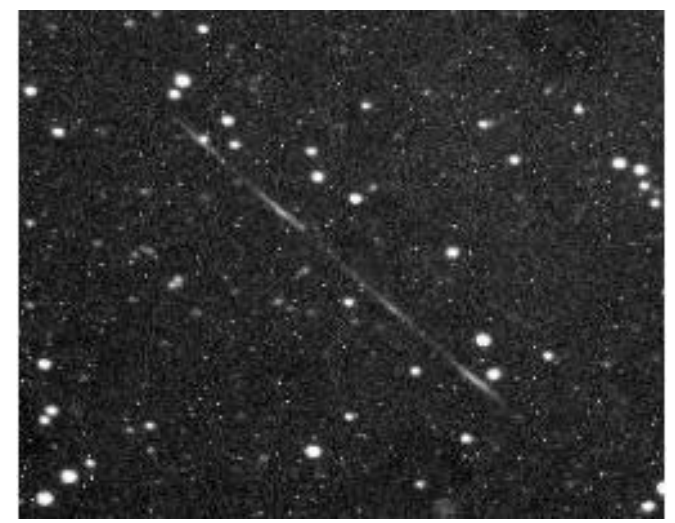

Figure 1. SL-6 (No. 13016) ${ }^{[11]}$.

The two brighter peaks are the side of the $S L-6$ and the two dimmer peaks are the ends of the cylinder. Image taken from Somers.

The observation technique used for their results was to record the target optical signature as a streak on a CCD image where the stars appear stationary. An example image is shown in Figure 1. These streaks show obvious dimmer and brighter spots in the image, and the length of each streak is related to the length of the exposure. The times at which the object produced a flash can be determined if the streak shows a clear start and end time, and the start time of the exposure and the length of the exposure is known. If the brightness of the target is plotted against time, then the plot is called a light curve. The flashes can then be taken directly from the light curve where the brightness is a maximum.

\section{A. Synodic and Sidereal Period}

As a cylindrical rocket body tumbles through space, it will reflect sunlight at an observer. To measure the period of the object's rotation simply requires analyzing how often a flash of light is seen from the object. The period of flashes as seen by an observer is known as the synodic period, and can also be described as the amount of time it takes the cylindrical object to make one full revolution relative to the Earth observer. However, the synodic period will change because the sun, cylinder, and cylinder geometry is constantly changing due to the rotation of the Earth, the movement of the object around the Earth in its orbit, and the movement of the Earth around the sun. Therefore, the sidereal period is defined as the amount 
of time it will take the cylindrical object to make one full revolution about its spin axis relative to a fixed direction (which is defined by the stars). The sidereal period is constant, and can be determined from the synodic period by analyzing the geometry of the situation as shown in Figure 2.
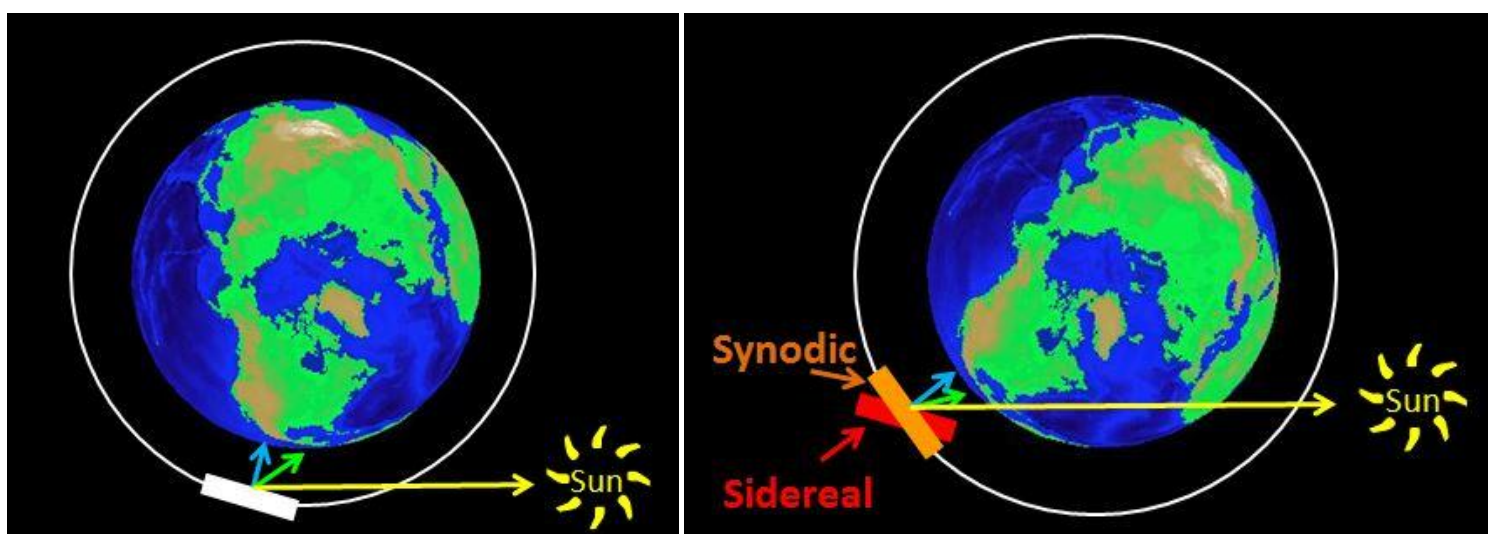

Figure 2. Illustration of Synodic vs. Sidereal Period. The left image shows a cylindrical rocket body at some time to start with. The light blue arrow is the object to observer direction, the yellow is the object to sun direction, and the green line is the angle bisector. The right image shows the same rocket body at some later time, where the red orientation is the same as the starting orientation relative to the sun, and is therefore a whole number of sidereal rotations later. The orange orientation shows the orientation as being the same as the starting orientation relative to the observer which defines the synodic period.

\section{B. A Summary of the Simplified Epoch Method}

The epoch method was originally developed as a technique for estimating the spin axis and rotation rate for asteroids and was later successfully used to determine the spin axis and rotation rate for cylindrical LEO satellites with synodic flash periods of about twenty seconds ${ }^{[11]}$. For more detail on the application to LEO satellites see De Pontieu ${ }^{[5]}$ and APPENDIX A: Application to LEO Satellites. Observation of LEO satellites was not the main objective of this paper for the reason that it would be very difficult if not impossible to track fast moving LEO satellites with the telescope and dome set up that was used.

To extend this method to GEO objects, some assumptions need to be made ${ }^{[14]}$. The first is that the object is a cylinder because their geometry is relatively simple compared to that of an asteroid of arbitrary shape. Next, assume the cylinder is in an end over end tumble, which is reasonable for uncontrolled rocket bodies. This is a reasonable assumption because the moment of inertia for a cylinder is the least around the body axis, and the greatest about any axis perpendicular to the body axis. With energy dissipation, an object will transition to spinning about the axis with the greatest moment of inertia. The third assumption is that the observer will only see the specular glints from the edge of the rocket body as it rotates. Finally, it is 
assumed that the spin axis and sidereal rotation period are constant, else it might be impossible to calculate this information.

To calculate the spin axis direction and the sidereal period, several vectors are needed: the vectors from the cylinder to the sun, from the cylinder to observer, the vector aligned with the long body axis of the cylinder, the spin axis vector about which the cylinder is tumbling (which is assumed perpendicular to the body axis), and the phase angle bisector, which is the vector that bisects the angle formed by the suncylinder-observer angle. The phase angle bisector is found by adding the unit vectors for the cylinder to sun and the cylinder to observer. In order to see a reflection or flash from a cylinder, the phase angle bisector must be perpendicular to the body axis vector. An image of these vectors relative to the rocket body is shown in Figure 3.
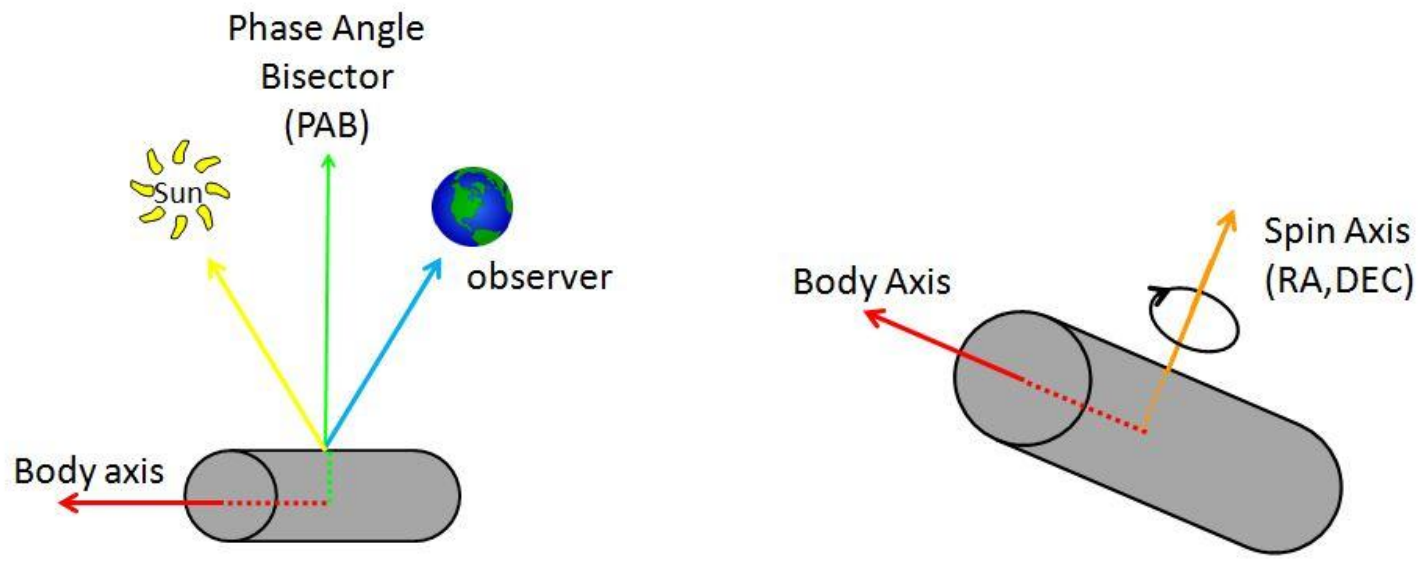

Figure 3. Visualization of Vector Directions. On the left, the cylindrical rocket body is shown with the body axis perpendicular to the phase angle bisector. This is the condition necessary to see a flash from the object. The phase angle bisector is found by adding the unit vector from the object to the sun with the unit vector from the object to the Earth. In the right figure, the spin axis is shown perpendicular to the body axis for end over end tumble.

There is no simple straight forward equation for the spin axis vector or the sidereal rotation period, so a brute force process is used. Every possible spin axis vector is tried, being defined as the unit vector in the direction of RA values from $0^{\circ}$ to $360^{\circ}$, and DEC values varying from $-90^{\circ}$ to $+90^{\circ}$. A visualization of Right Ascension and Declination values on the celestial sphere can be seen in Figure 4. The sidereal period is varied \pm 0.040 seconds away from the synodic period. With guesses for RA, DEC, and the sidereal period, a simulation is run that will find the necessary vectors for each time a flash is observed. The only unknown after making these guesses is the direction of the body axis of the cylinder. For this reason, it 
must be assumed to be aligned in such a way that the first observed flash is produced. That is, the body axis is defined as the cross of the spin axis vector and the phase angle bisector because the body axis vector is always perpendicular to the spin axis vector, and is perpendicular to the phase angle bisector at the time of

a flash. The first flash observed is therefore known as the reference flash.

To find which combination of values for RA, DEC, and sidereal period is the best, each candidate is ranked by a score that is calculated as follows during the simulation. First, because the body axis direction of the cylinder is calculated because it changes for each new spin axis direction. This is the reference body axis direction. At the time of the second observed flash, the new body axis position is found, and the angle between the reference body axis vector and the current body axis vector is defined as angle A. An illustration of the angle $\mathrm{A}$ is shown in Figure 5. Next, the time that has passed since the

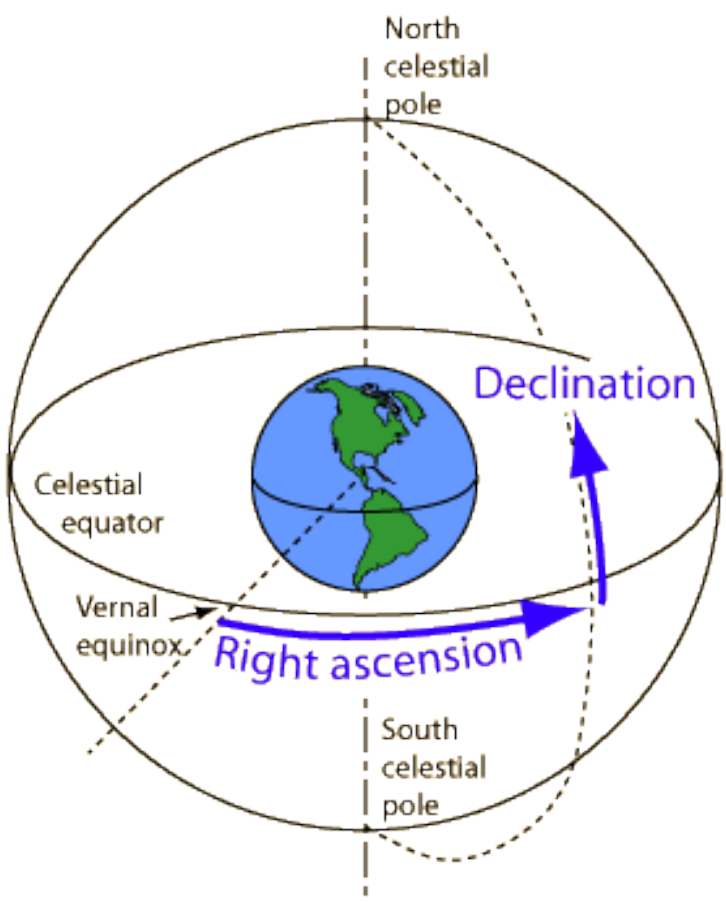

Figure 4. Right Ascension and Declination Image ${ }^{[8]}$. Right Ascension is measured counter clockwise along the plane of the Earth's equator, and starts at 0 in the direction of the Vernal Equinox. The declination is measured from the equatorial plane up (positive) or down (negative).

reference flash is divided by the sidereal flash period (which is half of the candidate sidereal period for each simulation) and the modulus remainder of a sidereal flash period is found ${ }^{1}$. To convert this into angle $\mathrm{B}$, the remainder is multiplied by $360^{\circ}$ and divided by the sidereal rotation period. If the candidate sidereal rotation period and spin direction are the actual sidereal rotation period and spin direction, then the difference between angle A and angle B will be zero. This process of finding A and B is repeated for each observed flash, and the score for that candidate RA, DEC, and sidereal rotation period is the sum of scores.

${ }^{1}$ An issue is introduced with this calculation, as the modulus remainder from this division could return negative numbers depending on the remainder function being used. It is understood that Somers has gone through multiple iterations of software since this paper was written and this may not be an up-to-date description of the process currently being used. 
This is repeated for every possible RA, DEC, and sidereal rotation period, and the candidate RA, DEC, and sidereal rotation period with the lowest score is then said to be the best fit for that object ${ }^{[11]}$.

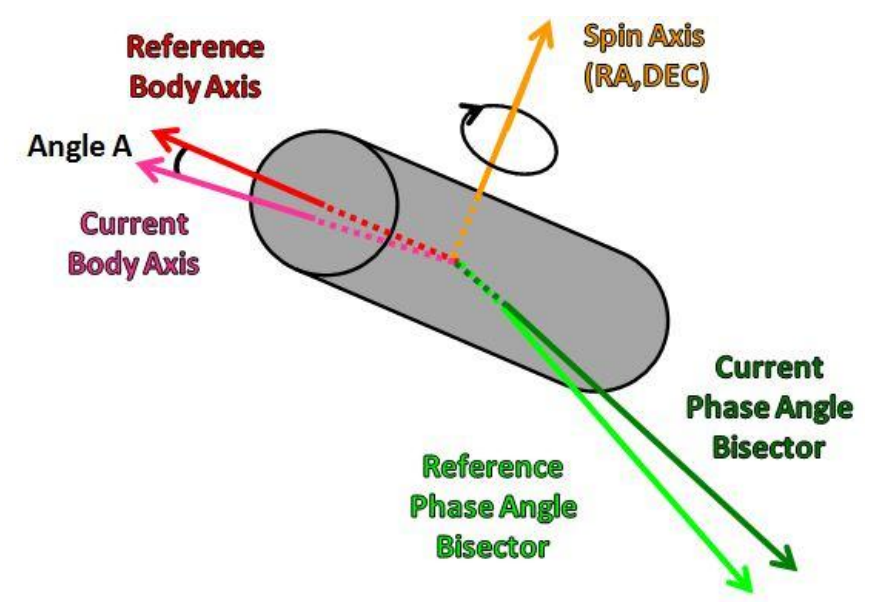

Figure 5. Visualization of Angle A of the Epoch Method. The reference body axis is the direction the body axis would be pointing to produce the first flash observed, and this is done with the first (or reference) phase angle bisector. For each subsequent flash, the current phase angle bisector for that time is crossed with the spin axis vector to produce a new body axis vector. The angle $A$ is the angle between the reference body axis vector and the current body axis vector for that observed flash.

This method works well when the phase angle bisector changes rapidly over time, as is the case for LEO objects, and as a result, short observations of fifteen minutes can be sufficient to get enough information on the object. However, for higher altitude objects near the GEO regime, the phase angle bisector does not change as quickly over time, and longer observations are needed (though not necessarily continuous $)^{[14]}$. For this reason, observations of objects were taken several images at a time, then about two hours later, a few more images were taken. This maximized the number of targets that could be observed in a short amount of time while still obtaining enough data to effectively calculate the spin axis of the object.

\section{The Ariane 5 Target}

The Ariane 5 rocket is a common launch vehicle for GEO-bound spacecraft. It can launch a single payload to a desired orbit, but more often than not, it is very popular for dual-payload geostationary spacecraft ${ }^{[1]}$. All of the rocket bodies observed for this research launched geostationary payloads, and all of the objects that were observed successfully were dual launches. The list of objects observed and their respective payloads can be seen in Table 1. For a dual launch, the "second" payload is attached to the lower payload attach fitting (PAF), and a cylinder shape with a cone top called a SYLDA is over it. The "first" 
payload is then attached to the PAF on the top of the SYLDA and the entire assembly is encased in the faring. A breakdown of the Ariane 5 can be seen in Figure 6 and Figure 7.

Table 1. List of Observed Ariane 5 Rocket Bodies.

\begin{tabular}{|c|c|c|c|c|c|}
\hline SSN & INTLDES & Launch Date & Launch Site & Payload 1 & Payload 2 \\
\hline 36034 & $2009-058 C$ & Oct. 29, 2009 & French Guiana & NSS 12 & THOR 6 \\
\hline 37777 & $2011-041 C$ & Aug. 6, 2011 & French Guiana & ASTRA 1N & BSAT-3C \\
\hline 37811 & $2011-049 C$ & Sept. 21, 2011 & French Guiana & SES 2 & ARABSAT 5C \\
\hline 39218 & 2013-038D & July 25, 2013 & French Guiana & ALPHASAT (I-4A F4) & INSAT 3D \\
\hline 39235 & 2013-044C & Aug. 29, 2013 & French Guiana & EUTE 25B & GSAT 7 \\
\hline
\end{tabular}

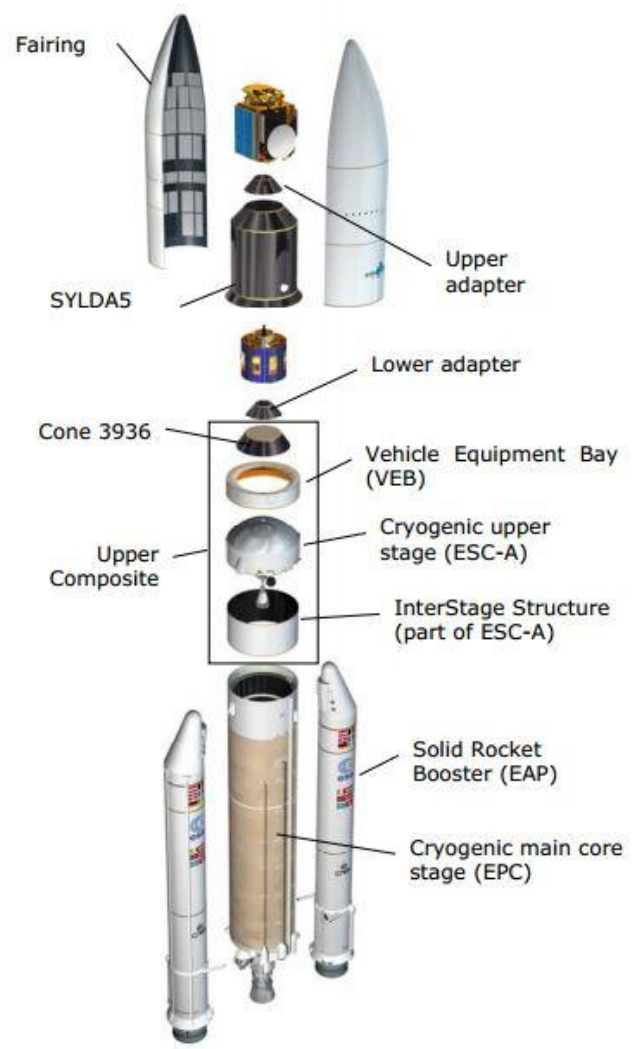

Figure 6. Ariane 5 Breakdown ${ }^{[2]}$. The rocket body that was observed is the upper composite section in the box. The SYLDA is also shown. The payloads are two objects that are not labeled.

For each of the observed targets listed in Table 1, the two payloads are listed with international designator letters A and B, while the remaining Ariane 5 rocket body and the SYLDA are listed with letters $\mathrm{C}$ and $\mathrm{D}^{[10]}$. In each case, the SYLDA is listed as debris (DEB) and the observed target is listed as a rocket body (R/B) but they do not have the same international designator letter each time. None of the launches 
left any other objects in orbit (whether debris, rocket body, or payload) therefore, the target that was observed must be the Ariane 5 upper composite ${ }^{[2]}$. The upper composite is the combination of the vehicle equipment bay and the cryogenic upper stage and is shown in the rectangle in Figure 6. Actual images of the upper composite and VEB are shown in Figure 8.

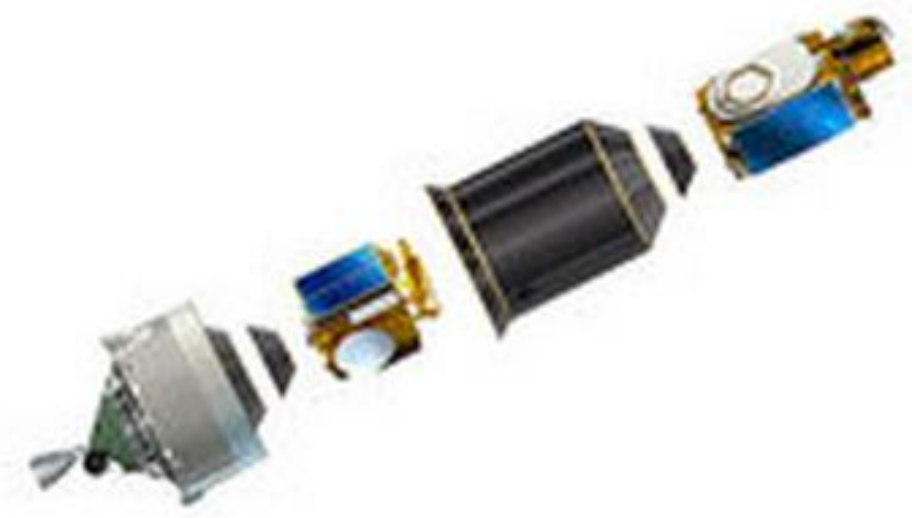

Figure 7. Diagram of Ariane 5 Upper Structure ${ }^{[6]}$. The upper composite is on the left, the first payload is then shown, followed by the SYLDA, and finally the second payload is on the right. 


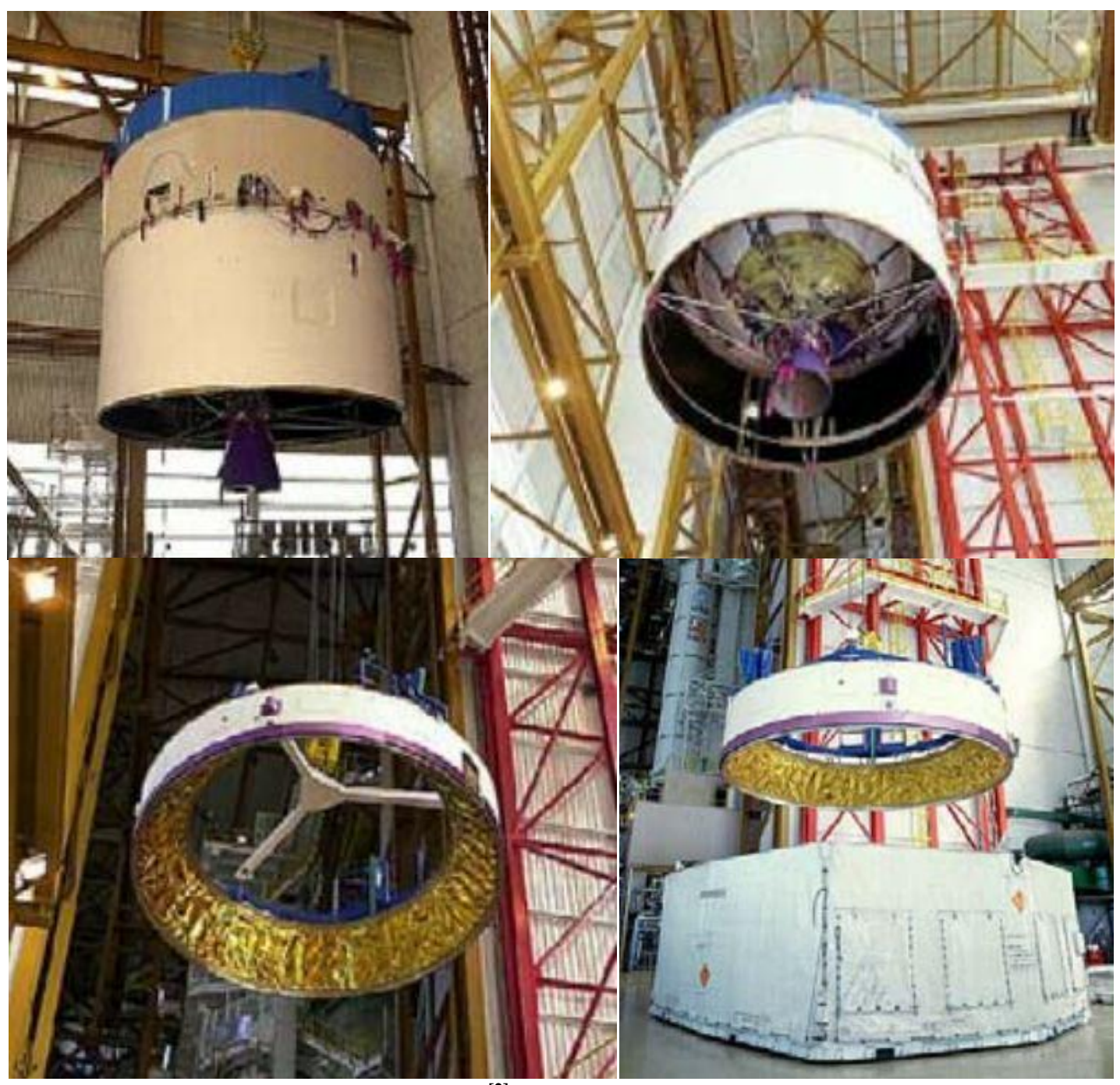

Figure 8. Ariane 5 Upper Composite Images ${ }^{[2]}$. Two different views of the Ariane 5 upper stage and vehicle equipment bay are shown. The upper stage (ESC-A) is in the top two images, and the VEB is in the two lower images. 


\section{INSTRUMENTATION}

The Cal Poly Observatory is a small dome that houses the brand new Meade LX600 that was installed in January of 2014. Images of the dome can be seen in Figure 9. The approximate observatory coordinates are $35.30^{\circ} \mathrm{N}$ and $120.66^{\circ} \mathrm{W}$ at $105 \mathrm{~m}$ above sea level. Other components include the camera, the focal reducer, focuser, the dome software/hardware, and other computer software including CCDSoft and the SkyX. Model numbers, serial numbers, and other information about the hardware and software used is presented in APPENDIX B: List of Components and Software Used. For this reason, the software versions are not listed here.

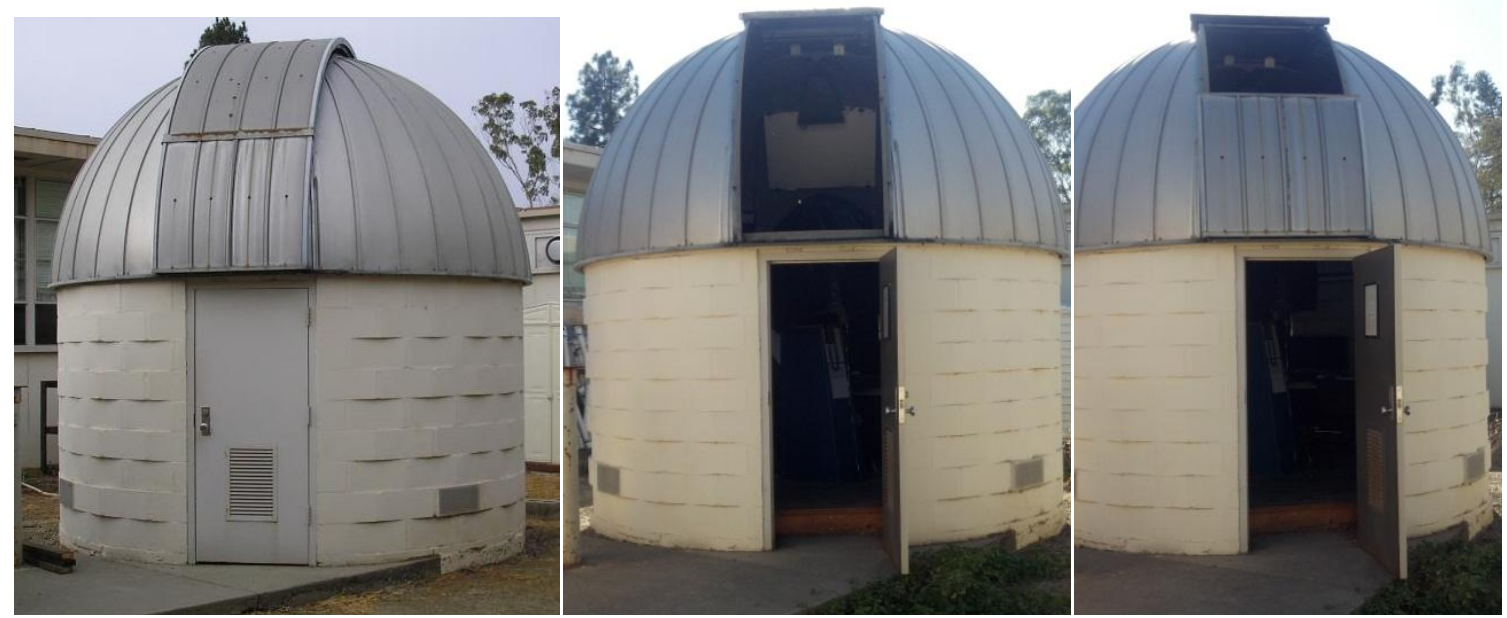

Figure 9. Images of the Cal Poly Observatory. Left: Dome shut. Center: Dome open with windbreak up. Right: Dome open with windbreak down. The placement of the windbreak can allow for low altitude observations or high altitude observations.

\section{A. Telescope}

The telescope used was a Meade LX600 14" that is mounted on the X-Wedge base. The specifications of the telescope can be seen in Table 2. When the camera, focuser, focal reducer, and CCD camera are all attached to the telescope, the setup can be seen in Figure 10. Due to the length of the focuser, focal reducer, and CCD camera, the telescope cannot point within $30^{\circ}$ of Polaris. Also, the windbreak on the dome was usually left down (shown in the far right image of Figure 9), which limited the telescope to objects at elevations of $30^{\circ}$ above the horizon. Even if the windbreak was raised, the buildings surrounding the telescope were still likely to block the same amount of sky as the windbreak depending on what direction 
the telescope was pointing. Regardless, there is considerably more airmass near the horizon making the light curves noisier so observations below $30^{\circ}$ of elevation were rare.

Table 2. Telescope Specifications.

\begin{tabular}{|l|l|}
\hline \multicolumn{1}{|c|}{ Specification } & \multicolumn{1}{c|}{ Value } \\
\hline Aperture & 14 inches $(355.6 \mathrm{~mm})$ \\
\hline Optical Design & ACF (advanced coma free) optics \\
\hline Focal Length & $2845 \mathrm{~mm}$ \\
\hline Focal Ratio & $\mathrm{f} / 8$ \\
\hline Plate Scale & 0.73 "/pixel (with focal reducer) \\
\hline
\end{tabular}
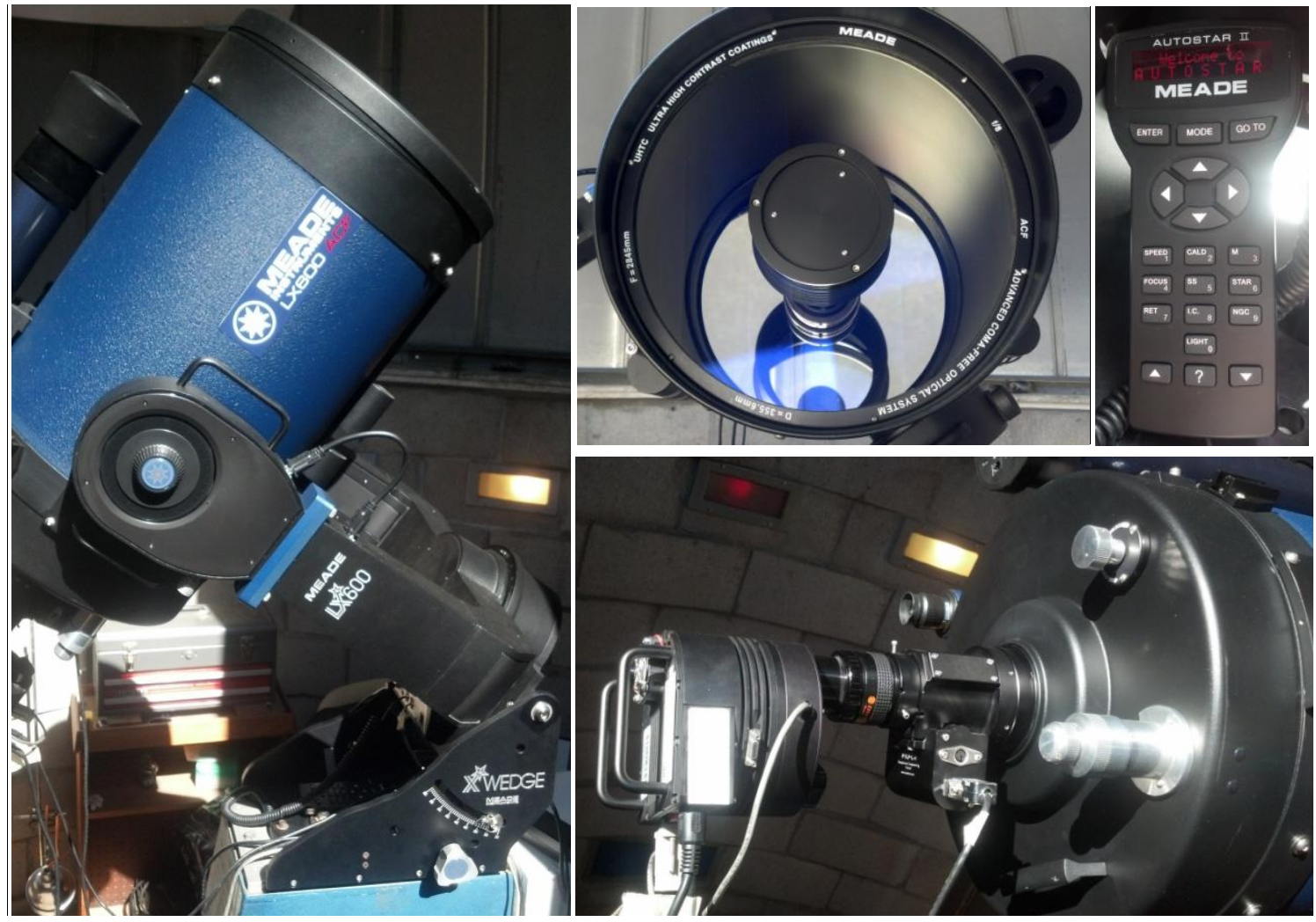

Figure 10. Images of the telescope. Left: telescope mounted on $X$-Wedge facing south in the home position. Top middle: looking down the telescope. Top right: keypad to enter the time and date, and is also used for centering objects in the finder scope. Bottom right, the back of the telescope, on which can be seen the focuser (closest to the telescope), the focal reducer (in the middle), and the CCD camera (farthest to the left in the image).

\section{B. CCD Camera and Focuser}

The CCD camera is a Santa Barbara Instruments Group Astronomical Instrument Camera and is seen in Figure 11. The pixel dimensions are 2184 by 1472 , where each pixel is $6.8 \mu \mathrm{m}$ and the total array is 
14.9x10 mm. The camera has multiple binning options, but for all science and calibration images, the $1 \times 1$ binning option was used for the highest quality images. Lower quality $3 \times 3$ binning reads out faster and was used for initial target searches. The focuser is a Temperature Compensating Focuser, and is used for fine control of the focus from night to night. The telescope focus knob is set to a rough focus value where stars are in focus within the range of the TCF, and is not changed unless the CCD camera is removed and a different camera or an eyepiece is installed. An image of the focuser can also be seen in Figure 11.

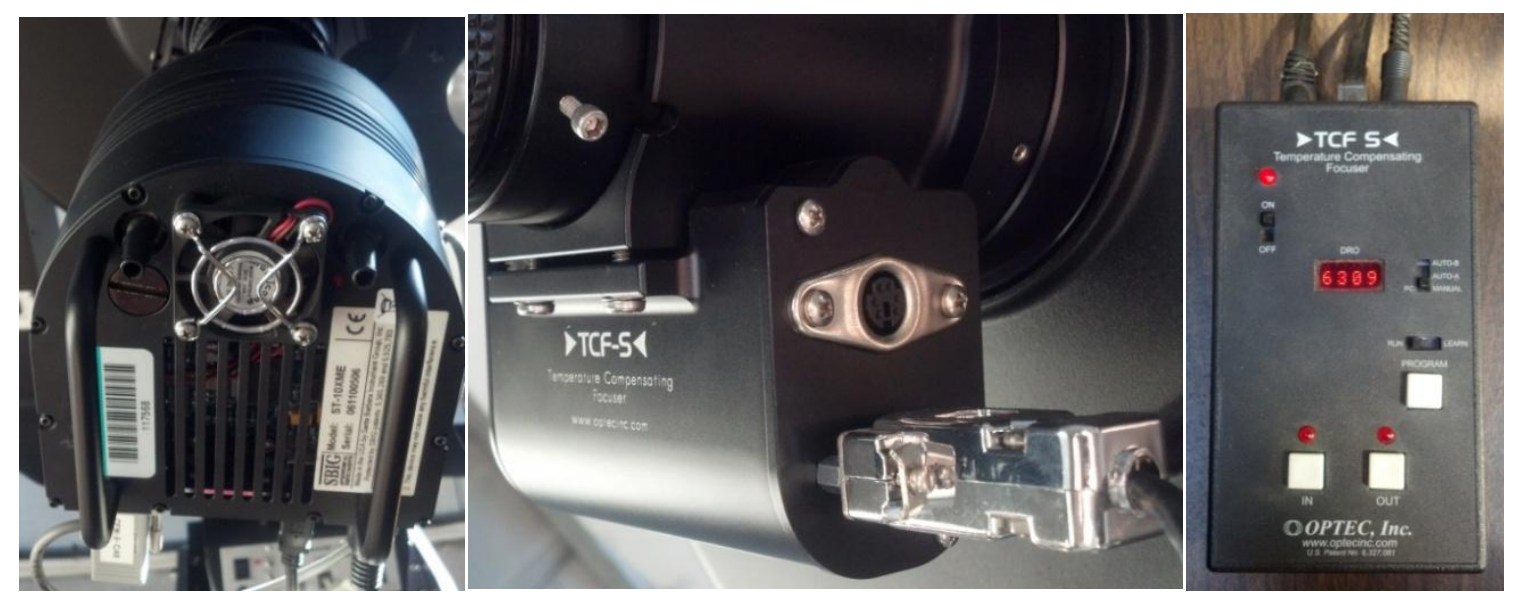

Figure 11. CCD Camera and Focuser. The left image is the camera, the middle image is the focuser, and the far right image is the focus controller box.

\section{Focal Reducer}

The focal reducer used is a Celestron $\mathrm{f} / 6.3$ (shown in Figure 12) and was used because it was available and provided benefits to looking for and tracking objects. Using a focal reducer yielded a slightly larger field of view, which aided in target acquisition. For example, one particular target was poised to go through the center of the image according to the SkyX, however, the cross track error was quite large from

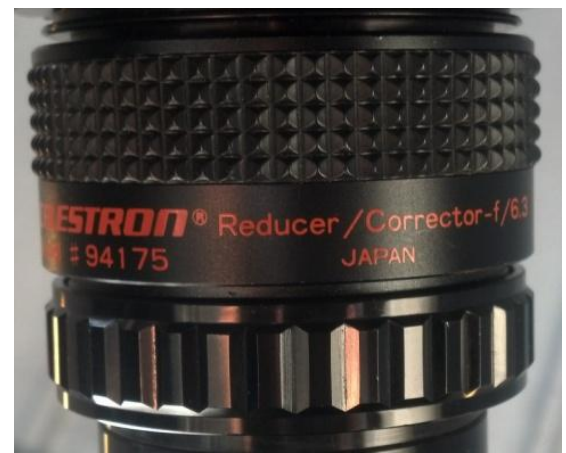

Figure 12. Focal Reducer.

Celestron model with a f/6.3 focal ratio. what the SkyX predicted, and the target was barely visible in the upper most corner of the CCD. Without the focal reducer, that target would not have been observed.

The focal reducer also allowed the observation of targets at slightly lower altitudes. This is because the criteria used for target observation was that the target take at least a minute and a half to cross the field of 
view. This ensured the target was moving slow enough for easy tracking with the telescope, and was the reason behind choosing to observe GEO or high altitude GTO objects. For very high altitude targets, the transfer time across the CCD image could take up to three minutes, and two exposures could be taken before moving the telescope again. Therefore because the focal reducer increased the field of view, it allowed targets moving slightly faster at lower altitudes to still meet the criteria of traveling slow enough across the FOV.

\section{The SkyX Program}

The SkyX software program is the telescope control software that was used for this project. It features a 3D celestial sphere on which is displayed the stars, the FOV of the camera, and where the telescope is pointed. In addition to this, the software also has the ability to display satellites from a TLE or 3LE file which was particularly useful. The telescope can be slewed to different parts of the night sky by clicking on any visible star and selecting the slew command. 


\section{OBSERVATION}

Each observation run consisted of several parts: setup, data collection, and shut-down. Set-up is what must be done before the telescope is used, data collection is the set of steps taken during observation to acquire images of the target(s), and shut-down is what must be done to ensure the telescope and other equipment is protected from night to night and will remain in good operational condition. Detailed steps for set-up and shut-down procedures that are specific to the Cal Poly Observatory are included in APPENDIX C: Cal Poly Observatory Telescope Set-Up and Shut-Down Procedures. General steps for set-up are still listed here as well as the process used for observing targets, and a flow chart of this process is shown in Figure 13.
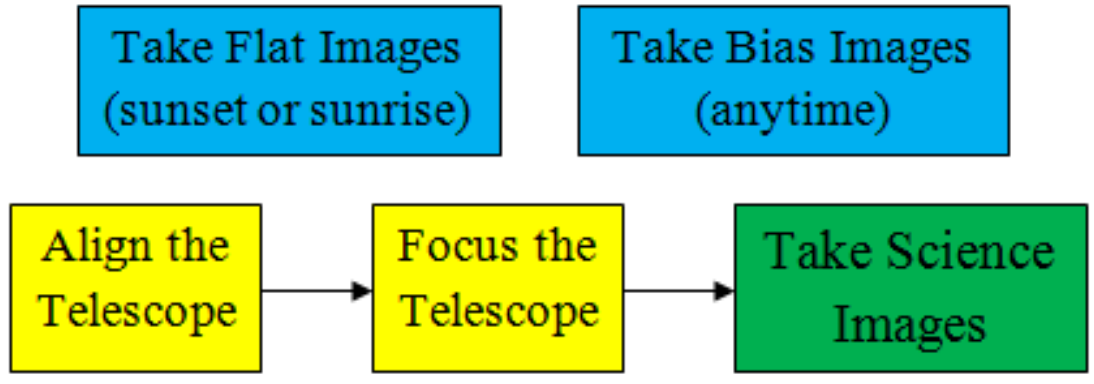

Figure 13. Flowchart for Data Collection. Calibration images are shown in blue. Pre-Data collection steps are shown in yellow. Science Images are green.

\section{A. Pre-Data Collection}

Once it gets dark enough to see a bright star, the telescope must be aligned. Alignment consists of pointing at a known bright star, and making sure it is in the center of an image when The SkyX thinks the telescope is pointing at that star. If the star is nowhere to be seen in the CCD image, the finder scope is used. The telescope constantly tracks at the rate the stars are moving, so whenever the telescope is disconnected/reconnected the tracking slightly misaligns the pointing. Once the bright star is in the center of the field of view of the camera, the SkyX is synced with that star so that the computer knows where the telescope is.

Focusing the telescope is done after alignment, and images were taken of a star close to magnitude six in brightness with camera exposures of three seconds. Images of the same star are taken over and over 
while the focus is adjusted manually using the focus controller box until a maximum brightness is reached. An in-focus star takes up less pixels on the CCD than an out-of-focus star, therefore when the light is concentrated in a smaller area, higher brightness values are achieved. However, due to fluctuations in the atmosphere, the value for the star brightness cannot always be trusted. A solution is to take several images before adjusting the focus again and to average the brightness at that focus value, or to examine the star in the image by eye and use the focus value that makes the star as small and round as possible.

\section{B. Data Collection}

Data collection is split into two parts, collection of calibration data and science data. Calibration data is composed of bias images, dark images, and flat images. Science data is the term given to the images of the target(s) being observed for the night because those are the images where the science comes from.

\section{Calibration Data}

Bias images are zero second exposures that are taken to eliminate noise added by the camera and are also known as "zeros". It is expected that if the camera collects photons for zero seconds, it would output that zero photons hit the detector, but in fact it displays a positive bias. This is because the CCD camera adds a bias voltage to the voltage recorded such that the analog to digital converter can register the voltage and accurately convert to a pixel value. For this reason, a bias image is subtracted from each dark, flat, and science image as the first step in data reduction. A visualization of data reduction can be seen in Figure 14.

Dark images are images that help eliminate thermal noise generated by the camera. Thermal noise is reduced by cooling the $\mathrm{CCD}$ to $-5^{\circ} \mathrm{C}$ but this does not get rid of all the thermal noise. For this project, the exact pixel counts are not important, because only the periodic motion of the light curve is analyzed. The actual or instrumental magnitude of the satellites is not necessary or used. For this reason, dark images were not taken. Normally, darks are taken with an exposure time equivalent to the science data images, but the shutter remains closed. The thermal noise dark images eliminate is proportional to the length of the exposure. For excessively long exposure times, dark images can be scaled. Dark images would be subtracted from each of the flat and science images as the second step in data reduction, but as they would only get rid of minimal thermal current, they were not used. 


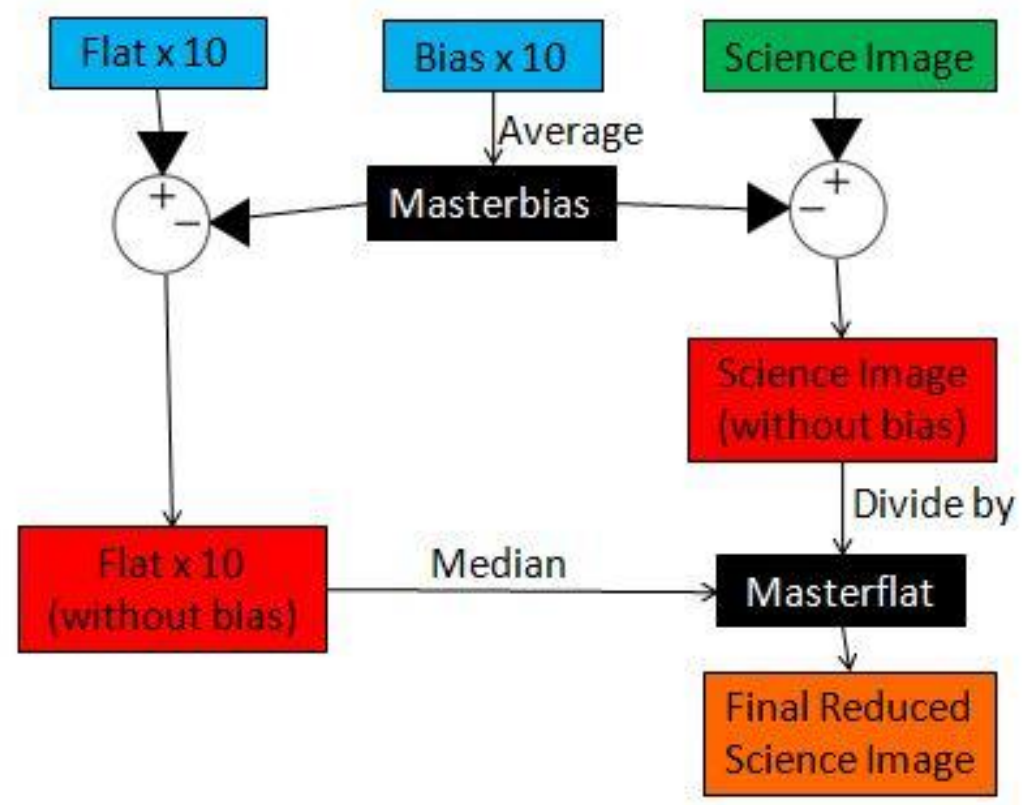

Figure 14. Flowchart of Data Calibration/Reduction. Bias images are combined using an average, then subtracted from all other images. The bias-corrected flat images are combined using a median and the science images are divided by the resulting masterflat image. This yields final calibrated science images.

Flat field images are taken of the sky just after sunset or just before sunrise. The idea behind the flat field image is to evenly illuminate the CCD camera to see what the pixel response is for the camera. The camera response is likely not flat, meaning that for the same amount of photons, one pixel might output a higher brightness value than another pixel. Once the flat field image is obtained, then the relative scales of each pixel are known. If a science image is divided by the flat field image, then the variations in the camera response for the image will be removed, and the new brightness values will be standardized across the entire image. The camera response is not just different for different pixels however, but it is also different for different filters. If data is taken in more than one filter then more than one set of flat field images needs to be taken. For all the data taken in this project, a clear filter (no filter) was used to allow the maximum amount of light, so only one set of flats was taken. The final step in data reduction is to divide all appropriate science images by a normalized flat field image of the same filter.

About ten pictures were taken each night of both bias and flat images, and each set was combined to create what are referred to as masterbias and masterflat files, respectively. An example of master files (including a masterdark) can be seen in Figure 15. Multiple bias images are obtained because there are noise and bias counts in the image, not just bias counts. If multiple images are used the noise can be 
averaged out by combining the bias images using an average. Multiple flat images are obtained for a similar reason, but instead of trying to average out noise, combining the flat images removes the effect of a star in the image. For this reason, the telescope is moved slightly between each image so the stars are not always in the same place. Because stars are much brighter than the background, the counts cannot be averaged like for the bias images, therefore the flats are combined using a median.
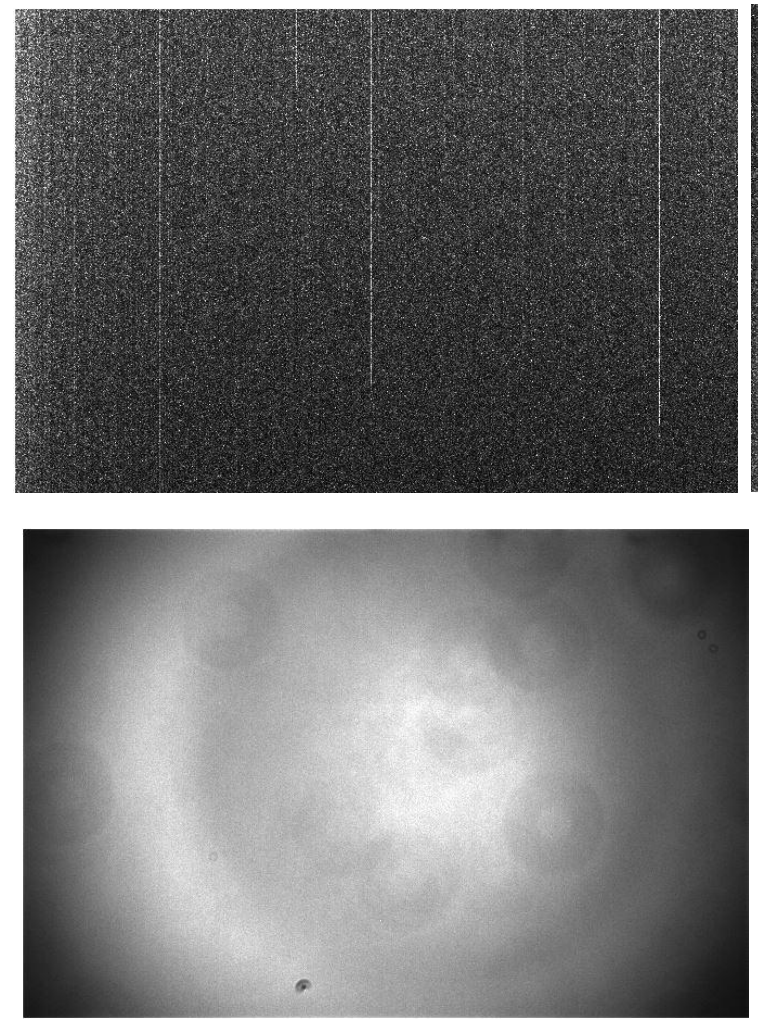

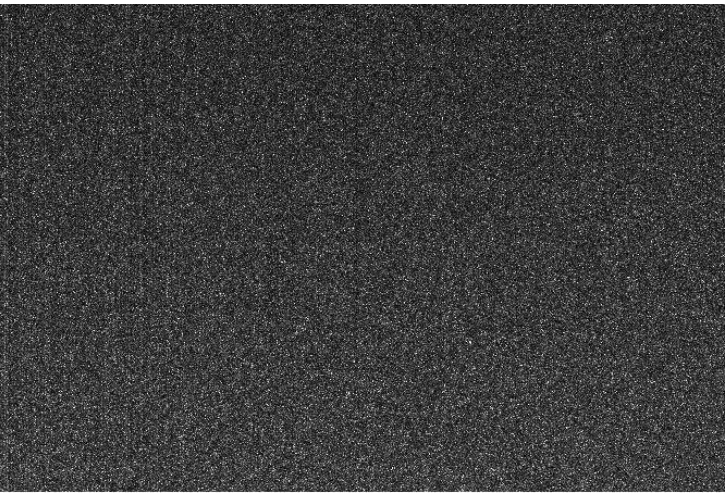

Figure 15. Bias, Dark, and Flat Images. These images from top left, to top right, to bottom left are examples of masterbias, masterdark, and masterflat images respectively. The bias and dark images are taken with a closed shutter, therefore nothing is visible in them except a few columns of bright pixels in the bias image. The flat field image shows the pixel response of the camera, which is high (bright) in the center, and low (dark) in the corners.

\section{Science Data}

The science data is the group of pictures that were taken of the targets of the observation run. For this research, the targets were an array of Ariane 5 rocket bodies. To begin, the website space-track.org was used to download three-line element sets (3LE data) for each satellite and was saved as a text file. The 3LE has the common name of the object on the first line (the zero line) and the two-line element data on the second and third lines (lines 1-2) which can be seen in Figure 16. The two-line element data (TLE) contains the orbital elements for the target at the specified epoch date in the file. This file was then uploaded directly into The SkyX where it will display the zero line of each 3LE next to the object, and will even display the NORAD number taken from the first line of the TLE information. The path of the target is also shown on the celestial sphere so its movement relative to the stars can be predicted for some number of minutes. 


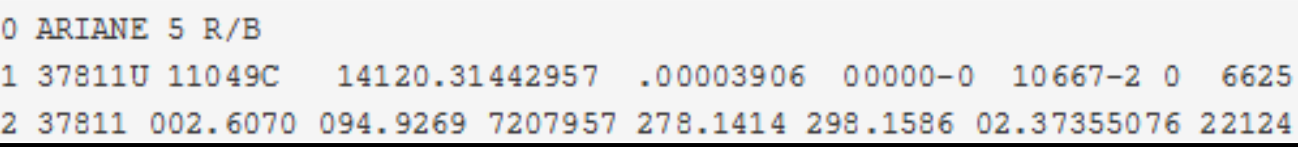

Figure 16. Example 3LE. The 3LE contains the 0, 1, and 2 lines. The TLE only contains the bottom two lines, the 1 and the 2 lines, but not the object name.

At this point, there are many short/long gray lines that represent targets on the celestial sphere, and the telescope is likely pointed at the focusing star for the night. To start tracking a target, the telescope is slowly slewed across the night sky toward the target, and re-centered on bright stars along the way. This is best done in about $\sim 30^{\circ}$ increments for this particular telescope because the tracking is not great for large slews (this issue is discussed further in APPENDIX C: Cal Poly Observatory Telescope Set-Up and ShutDown Procedures). Once the user has slewed near the location of the target with the telescope, it can still be a challenge to find the satellite. The SkyX is not perfectly accurate in its placement of the objects, and as a result, the object will likely NOT be exactly where The SkyX says it is. From trial and error, the author has found the satellite to pass through a given location a few minutes behind when it was predicted to pass through the field. The cross track error is usually not very large because the satellite generally follows the predicted path fairly well. A select few of the targets have had errors in the cross track direction, but it is usually still in the field of view of the telescope. The along-track error can be off by several minutes causing the satellite to appear "late." A further discussion the satellite along-track error can be found in APPENDIX C: Cal Poly Observatory Telescope Set-Up and Shut-Down Procedures. An image of the SkyX program with a target in view is shown in Figure 17.

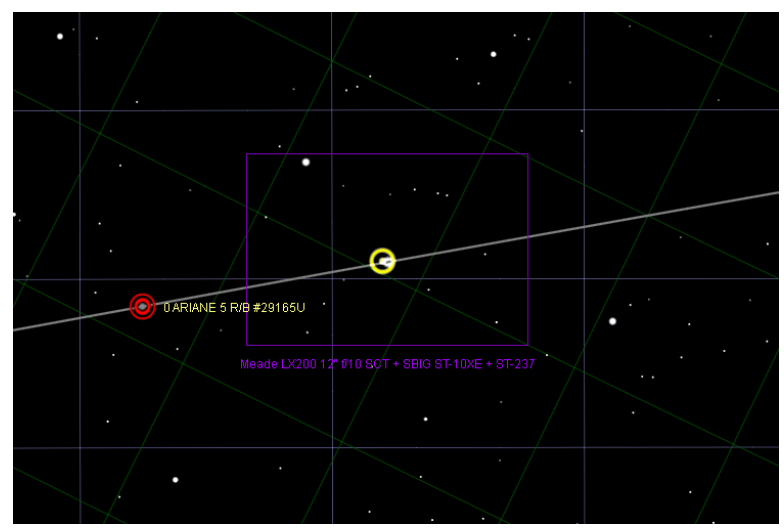

Figure 17. Image of the SkyX screen. The FOV of the telescope is shown as the purple box. The telescope is centered at the location of the yellow target in the center. The red target shows the last place clicked on the screen, in this case, it is on the Ariane 5 rocket body being observed. The SkyX will display the rocket body as a small gray circle moving along the gray line which is its predicted path. The green lines in the background are azimuth and elevation lines, while the gray lines show RA and DEC. 
The steps implemented to find targets were as follows: slew to a point along the object's path in front of where it "currently" is according to the computer. Using the focuser tab in CCDSoft (because the focus images are not auto-saved) the camera is set to take low-quality short-exposures until told to stop. This creates a low frame rate video of sorts, and this is watched until the target is spotted. Most of the Ariane 5 targets were bright enough to be spotted moving across the image with a 1-2 second exposure time. While waiting for the target to appear in the image, the time the gray dot passes by a star in the SkyX is noted, and the time the target actually passes by that star in the images is also noted, which allows the calculation of how far the satellite is behind. The easiest way found to track the target is to position the gray dot in the SkyX in the purple field of view box where the target appears in the image, and sync the telescope with the center of the FOV box. This means the target will appear in the image where it is expected to appear, but the stars in the image will not match the stars shown in the SkyX. A more complicated method of tracking would be to convert the time the target is behind into days, and add this decimal to the TLE file, however, this proved more difficult and not as effective.

Once the telescope is synced on the target, it is easy to follow it manually with the telescope. Because near geostationary objects were being observed, a one-minute exposure, or sometimes even two one-minute exposures can be taken before the target leaves the field of view of the CCD. Once the satellites are no longer in the image, the telescope can be slewed along the gray path line in the direction the target is moving, and is recaptured in several more images. Sets of ten images were taken at a time, and on good observing nights, two sets of ten images each were taken of the same target, but spaced apart by an hour or more. This allows the phase angle bisector to change significantly during this time, thereby reducing the error in the sidereal period and spin axis calculations. This is because the synodic period of the object will change as the phase angle bisector changes, and the more it changes during the observation, the more accurately the spin axis vector can be determined. If the phase angle bisector does not change enough throughout the observation, then the spin axis vector cannot be accurately determined. 


\section{IRAF DATA REDUCTION}

There are two main steps to data reduction, file type conversion, and calibration of the images. All data reduction was performed using a terminal to run ds9 and IRAF programs. A visual of data reduction can be seen in Figure 18.

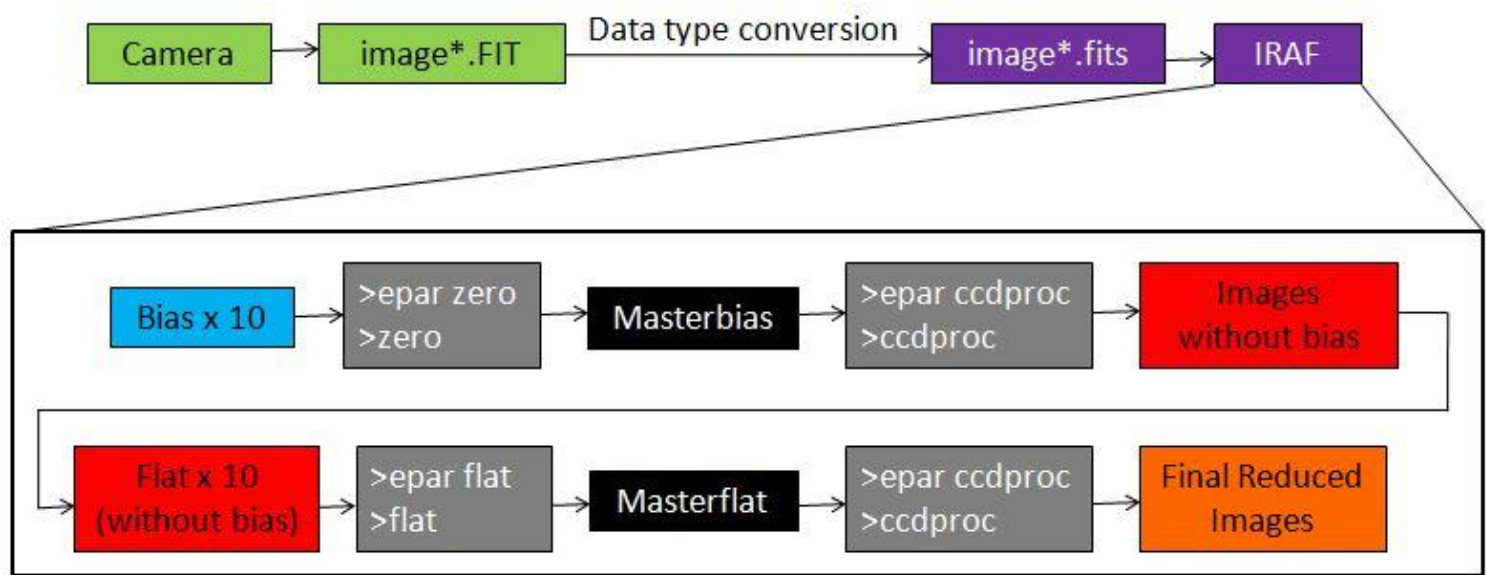

Figure 18. Visualization of IRAF Image Reduction. The CCD camera outputs *.FIT files which are converted to *.fits files that IRAF can process. IRAF averages the bias images using the zero command, then subtracts them from the remaining images using the ccdproc command. Using epar allows the user to adjust the command settings before running the command. The flat images are then combined using a median with the flat command, and the science images are divided by the masterflat using the ccdproc command.

\section{A. Data Type Conversion of Images}

The raw CCD files (*.FIT) need to be converted to the file type used by the IRAF software package (*.fits). This is very simply done using the rfits command found in the dataio package of IRAF. Using the command epar rfits allows the user to adjust the rfits parameters before calling rfits to do the file type conversion. The rfits parameters used are listed in Table 7 in APPENDIX D: Epar Settings Used in IRAF.

One unforeseen benefit to what seems like such a simple process is that it forces the user to make a copy of all the raw observation data before doing any data reduction. This way, if the data reduction process is messed up in some way, the * fits images can be deleted, and the process can begin again using the raw *.FIT files. 


\section{B. Calibration}

The ds9 program is used to view images throughout this process. The IRAF package used is in the directory: noao, imred, ccdred. Calibration of the science images then has several steps for each type of calibration image (bias and flat). The steps for bias and flat calibration are very similar, and will be

discussed at once. During actual calibration, all bias related steps are performed before any flat related commands are entered.

First, adjust the settings for the zero or flat command (epar zero or epar flat) using the values in the appropriate column of Table 8 in APPENDIX D: Epar Settings Used in IRAF. To combine the bias or flat images, run the zero or flat command. Once IRAF has finished making the masterbias image or masterflatClear image (the word "Clear" is added by IRAF to denote the filter with which the flat images were taken with), the images are viewed in ds9 before continuing to ensure they look acceptable. Next, edit the parameters of the ccdproc command (epar ccdproc) using the values in the appropriate column of Table 9 in APPENDIX D: Epar Settings Used in IRAF. Once this is complete, run the ccdproc command which will subtract the masterbias image from the flat and science images (for bias reduction) or divide the science images by the normalized masterflat image (for flat field reduction). If more than one filter was used during observation, then more than one set of flats should have been taken, and the flat reduction process will need to be completed multiple times for each filter used. In this research, only the clear filter was used. 


\section{IMAGE ANALYSIS}

After the image calibration was performed using IRAF, the image analysis was performed using selfwritten Matlab code in a multi-step process. The first step allowed the user to manually extract light curves from individual images, the second step allowed the user to analyze the light curve and manually determine where the flashes happen, and the final step uses the flash periods and a TLE to automatically calculate the best candidate sidereal period and spin axis. A visualization of this process is shown in Figure 19.

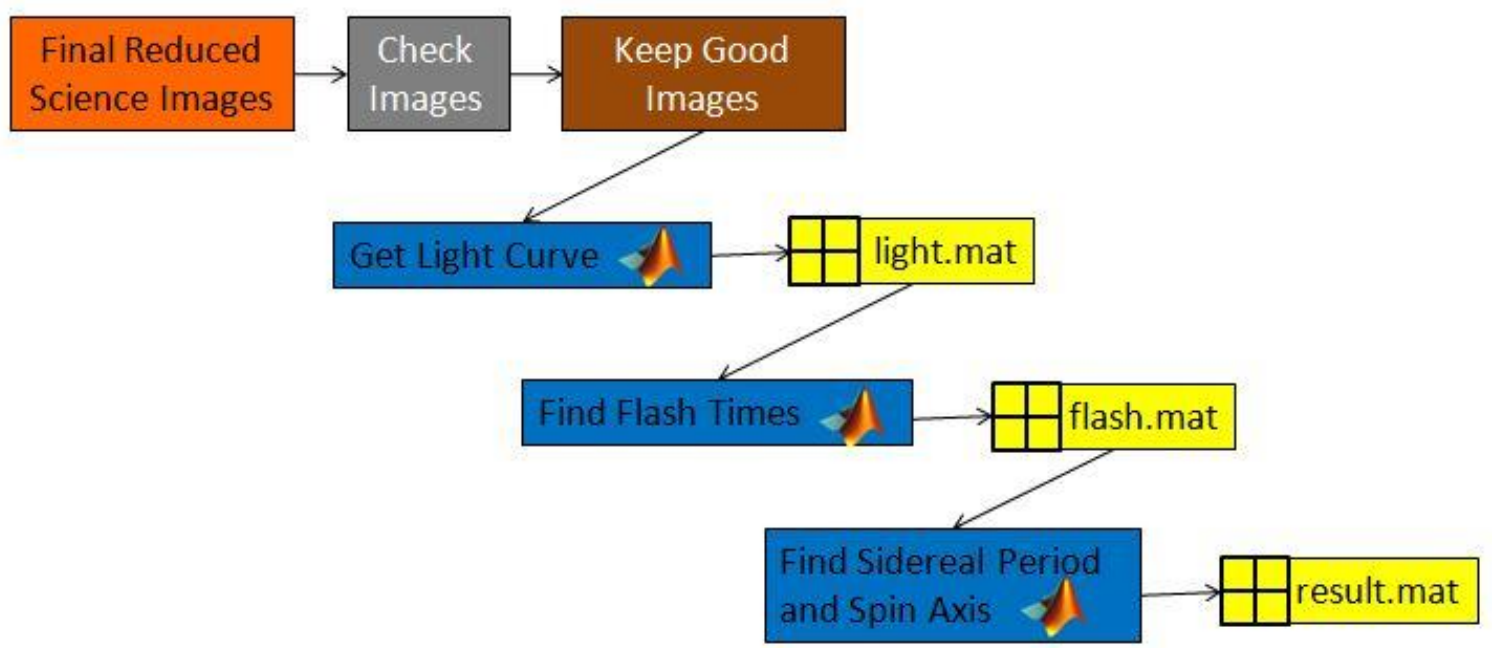

Figure 19. Visualization of Matlab used for Image Analysis. The output images from IRAF were looked at in $d s 9$ and/or Matlab, and good images were kept. Then, the light curve data was extracted from the images and saved as a Matlab data file. This file was used to find the flash times, and another data file was generated. The second data file was used to find the sidereal period and spin axis, and the resulting $3 D$ matrix was stored in a final Matlab data file.

\section{A. Checking Images}

The first thing completed for data analysis was to remove bad images from the data sets. Most of this was done during data collection, and an example of an image that was deleted during data collection can be seen in Figure 20. However, a few bad images still remained because some images were not deleted despite not being great images. If several images were taken where the stars appeared extremely blurry, these were deleted immediately, however, an image following these where the stars were still blurry, but not as blurry, 
was then sometimes kept because it was good by comparison. An example of an image that was kept vs. and image that was thrown out after data reduction is shown in Figure 21.

Another example of images not being deleted during data collection occurred if several images were taken of an object despite the object being very dim, or if images were taken of a final target for the

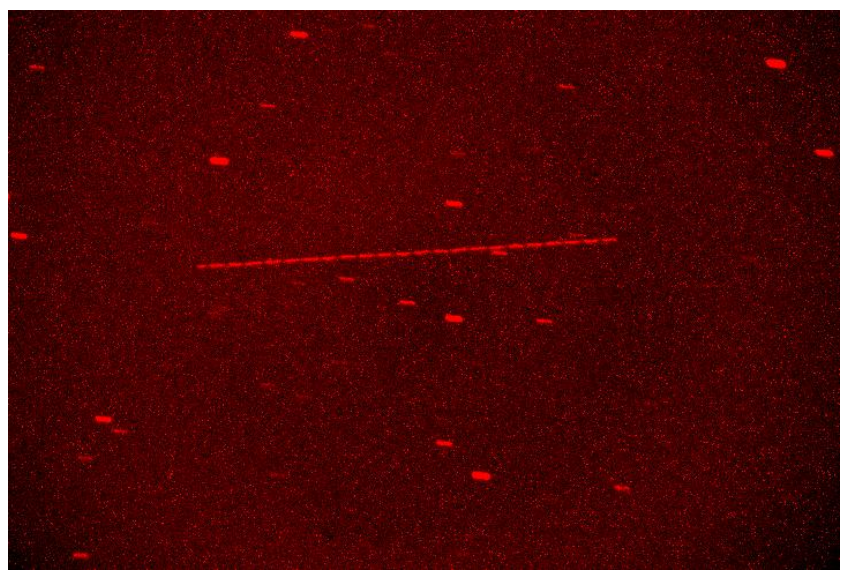

Figure 20. Image thrown out during data collection.

The image appears red because the observatory computer was in "night-mode" while observing. Blurred stars are evident, because the telescope had problems pointing. night, but it was starting to get light outside (the Sun would be rising soon) and the images were therefore not good to use. These images were not deleted on the spot to accelerate the process of observing the next target or closing up the observatory for the night, but a note was made in the log book to either look through these images more closely at a later time or to simply delete the specified images.
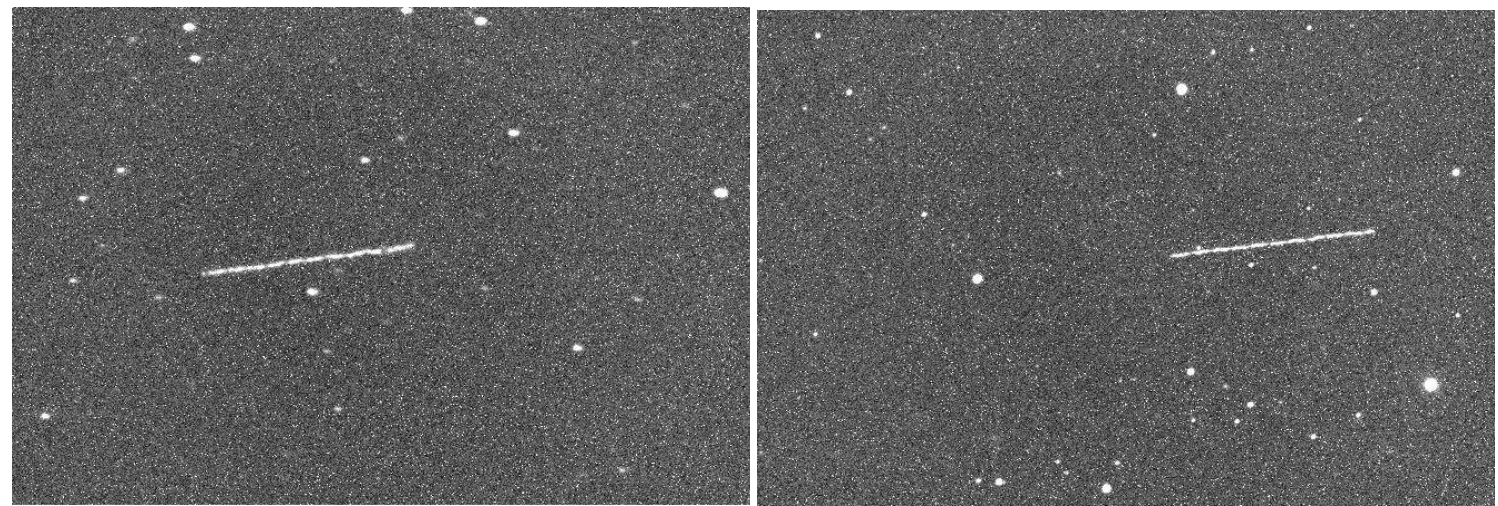

Figure 21. Good and Bad Data. On the left is an example of an image that was not used for image reduction. The object appears "fuzzy" on the right side and the stars are also blurry. Displayed on the right is an image that was kept. The stars appear circular as they should.

\section{B. Generating Light Curves}

When running the code, the user chooses each image file for analysis one at a time. Once the first image is selected, the image header information is read to determine the date and time of the beginning of the exposure, and the length of the exposure for the image. Next, the image is scaled using a logarithmic scale so the target is easier to see in the image display (scaling is for viewing only, the actual image data is saved for taking values from). For the first image, and every few thereafter, the user selects a section of the 
sky background to use in magnitude calculations. This must be done manually because it is not possible to hard code the program to use a section of the image that will not contain a star. After that, the target is zoomed in on (which appears as a streak across the image due to the minute long exposures), and the user selects two points beyond the edges of the target to create a cross section along the length of the streak. Once this is done, the light curve is created by averaging the brightness of pixels perpendicular to the user drawn line. An example image of this is shown in Figure 22 along with the generated light curve.
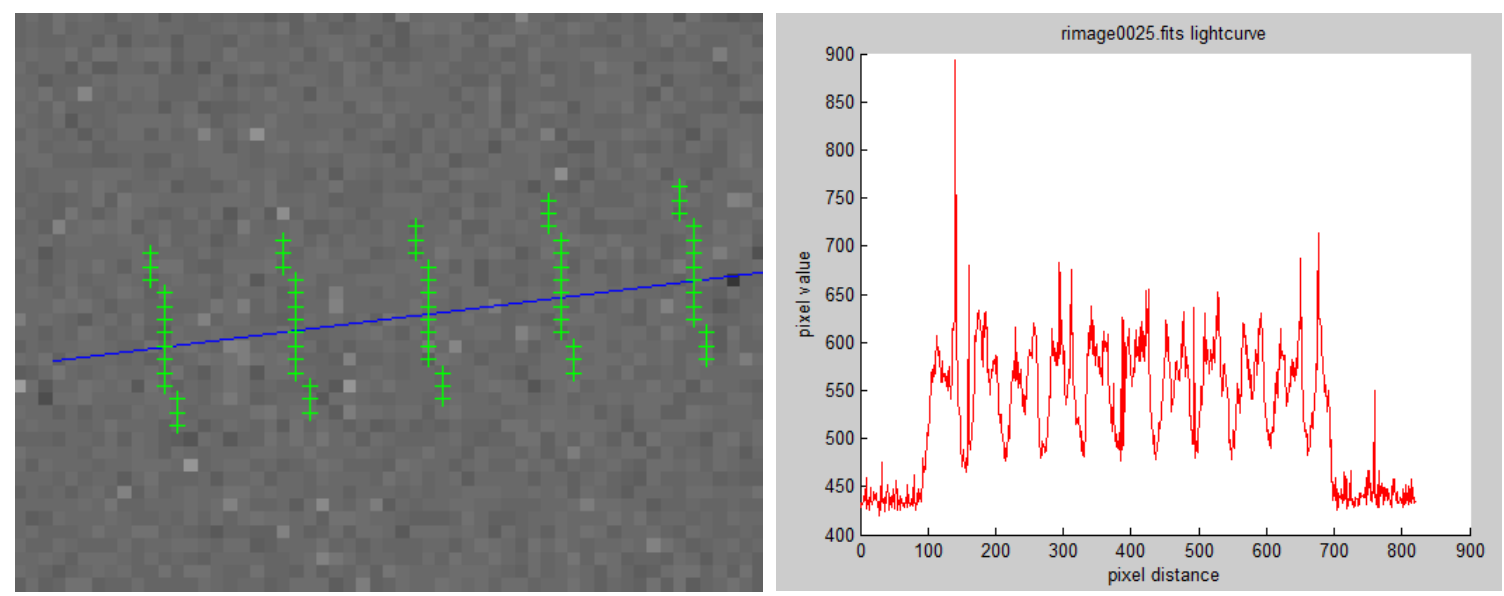

Figure 22. Summing Pixels to get a light curve. Left, close zoom in on the image, this shows the end of the user defined line that is over sky background. The pixels being averaged at each step along the blue line are shown in green and are as close to perpendicular to the blue line as can be achieved by rounding to a whole pixel. The green lines shown are spaced 10 pixels apart and only represent $1 / 10^{\text {th }}$ of the pixels being included in the light curve. On the right is the light curve corresponding to this image (only the far left part of the graph between about 0 and 55 pixel distance can be seen in the left image). The ends have lower counts because they are just sky background, and the edges of where the streak starts and stops are clearly seen. This is important because it allows the x axis to be transformed from pixel distance along the image into a Julian date.

Once the light curve graph is shown, the user manually selects the points at which the target streak begins and ends, which is how the beginning and end locations of the exposure are determined. The light curve data is taken from the section within the points the user clicked on, and flipped from left to right, because the target travels from right to left in the images. The date at each point along the light curve is mapped linearly to the pixel distance: the start of the light curve is the exposure date, and the end of the light curve is the exposure time added to the exposure date. After this, the file window allows the user to select another image and repeat these steps for all the images of one target. Upon completion, the entire light curve will be pieced together using the date, and the user is given the option to delete points above a 
certain threshold value. This is done to eliminate bright pixels and/or possibly bright stars that the target passed in front of. An example of this can be seen in Figure 23.

Finally, the pixel counts are transformed into instrumental magnitude using Eqn. (1):

$$
\text { instrumental magnitude }=C-2.5 * \log \left[\mathrm{N}_{\mathrm{star}}-\mathrm{N}_{\mathrm{sky}}\right]+2.5 * \log (t)
$$

The $\mathrm{N}_{\text {star }}$ value is the pixel value at each point along the light curve, and because these values are averaged across the green lines of Figure 22 they represent the counts for one pixel. The $\mathrm{N}_{\text {sky }}$ background value is calculated each time the user selects a sky background area on an image by averaging the pixel counts within that region. If more than one $\mathrm{N}_{\text {sky }}$ sample was taken for a set of images, the values were averaged to get an $\mathrm{N}_{\text {sky }}$ value for the whole set of images and this was done because the value does not vary much over the course of about twenty minutes. The $t$ is the length of the exposure, however, what that means for this situation is the amount of time the object spent illuminating one pixel. Therefore the amount of time the object spent over each pixel was calculated by dividing the exposure time by the number of pixels that were covered
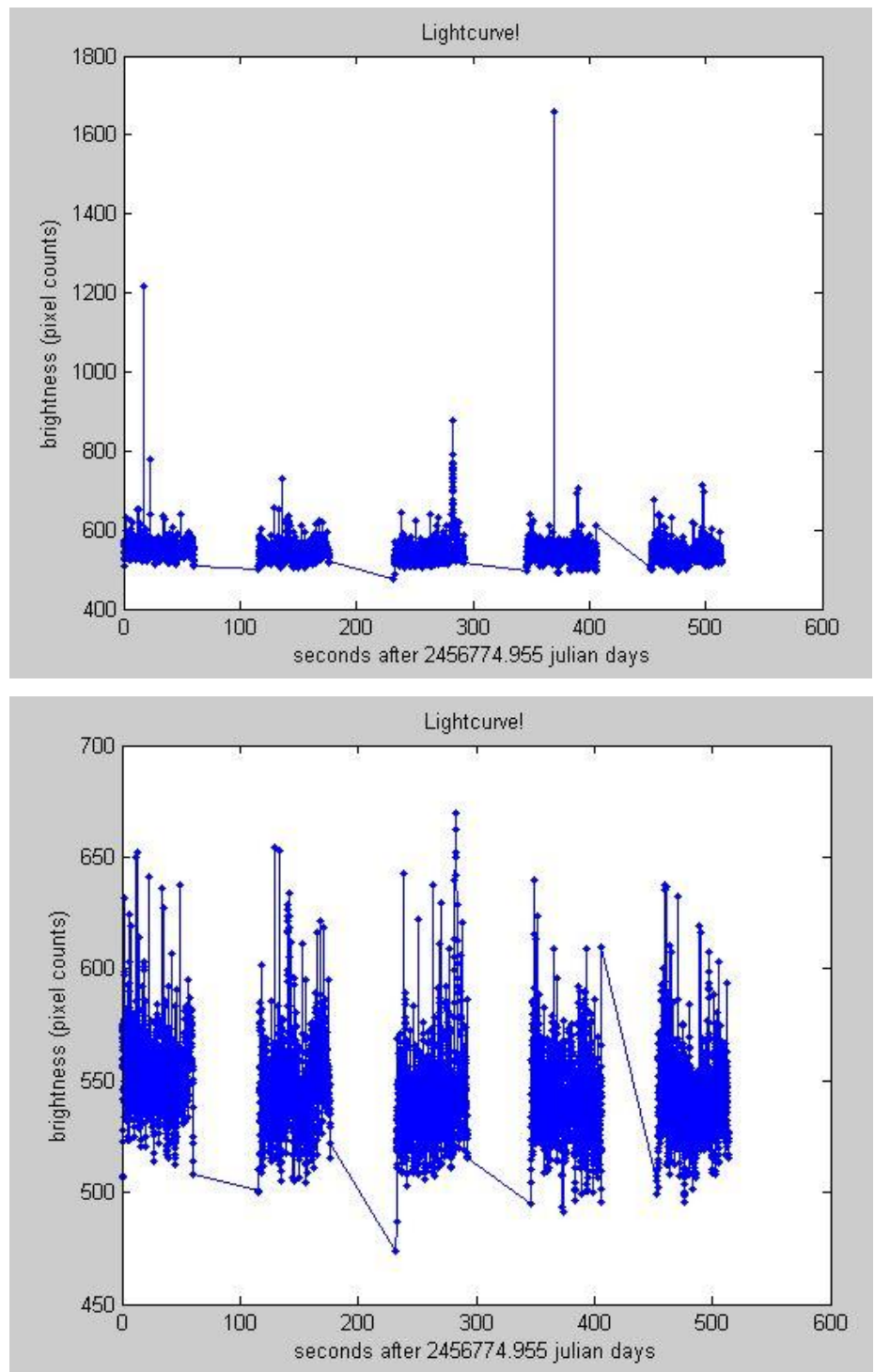

Figure 23. Example of Elimination of Bright Pixels.

The top is the before image, where there are obvious bright pixels that are much brighter than the actual object. The image can be zoomed in on for the user to decide on a value to use as a threshold, and data points above that brightness are eliminated. This results in the light curve seen on the bottom. For this observation, a value of 675 was used as the threshold. 
and used as t. The $t$ value was calculated for each image using the hypotenuse distance of pixels that were traveled across, but these values were averaged and one value was used for the entire data set. For data sets with large gaps of time in between, the beginning images were analyzed separately from the later images so that different $\mathrm{N}_{\text {sky }}$ and $\mathrm{t}$ values were used for the each set, and the light curves were pieced together afterward. An example of a light curve plotted in pixels vs. plotted in instrumental magnitude is shown in Figure 24. It is important to note that in the pixel scale, the brightest part of the light curve has the most counts, however, in the magnitude scale, the brightest points on the light curve are the lowest numbers.
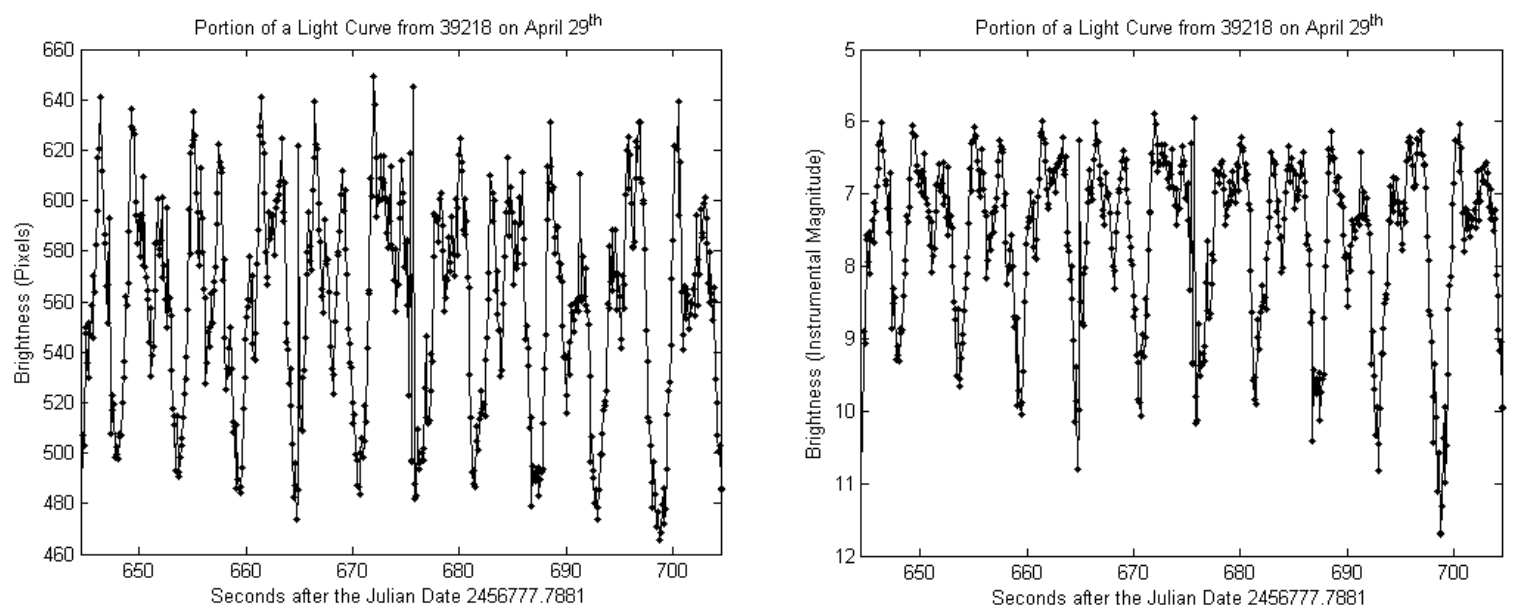

Figure 24. Light Curves in Pixels vs. Instrumental Magnitude. The top image is plotted with CCD pixel brightness on the $y$ axis, therefore higher values are brighter. The bottom image has instrumental magnitude on the y axis, therefore lower numbers are brighter.

The only part of the equation left is the $\mathrm{C}$ value, which is impossible to calculate without a filter. The clear filter was chosen to allow as much light in as possible because the rocket bodies were expected to be dim. For this equation, a value of 24.9921 was used, and was calculated as follows: the experimental brightness of fourteen stars was calculated using IRAF (package images $>$ tv). The epar rimexam setting of magzero was set to 0 because that is the $\mathrm{C}$ value that IRAF uses. The imexam command was then used on each star to find the magnitude without the $\mathrm{C}$ value. The "actual magnitude" of these stars was taken from the SkyX catalog where the "Magnitude" is listed, which is separate from where the B magnitudes and the V magnitudes are listed. The sum of squares error for various $C$ values was found using Matlab, and the $C$ value that yielded the lowest error was chosen. Again, this is not the correct way to calculate the $\mathrm{C}$ value because star magnitudes are only defined with a filter, and the $\mathrm{C}$ value is different for each filter. All of the light curve data that was used for further analysis is presented in APPENDIX E: Selected Light Curves for 
Analyzed Data, and selections of light curves from individual images are also presented for each set of images taken.

\section{Determination of Flash Times}

The next step is to load a specified file that contains data for the light curve that was created in the first step. The light curve for the entire night will be plotted so the flash times can be manually selected. The data for one set of observations is shown in Figure 25, and each blob represents one image that was taken with the telescope. After zooming in, the periodic motion of the light curve is apparent, and this can also be seen in Figure

25. Because the light curves had sharper minimums, it was assumed that the flash would occur centered between minimums of the light curve, and this was used to calculate the flash times. The user manually selects two adjacent minimums, and blue diamonds and green squares appear at those locations. The shapes
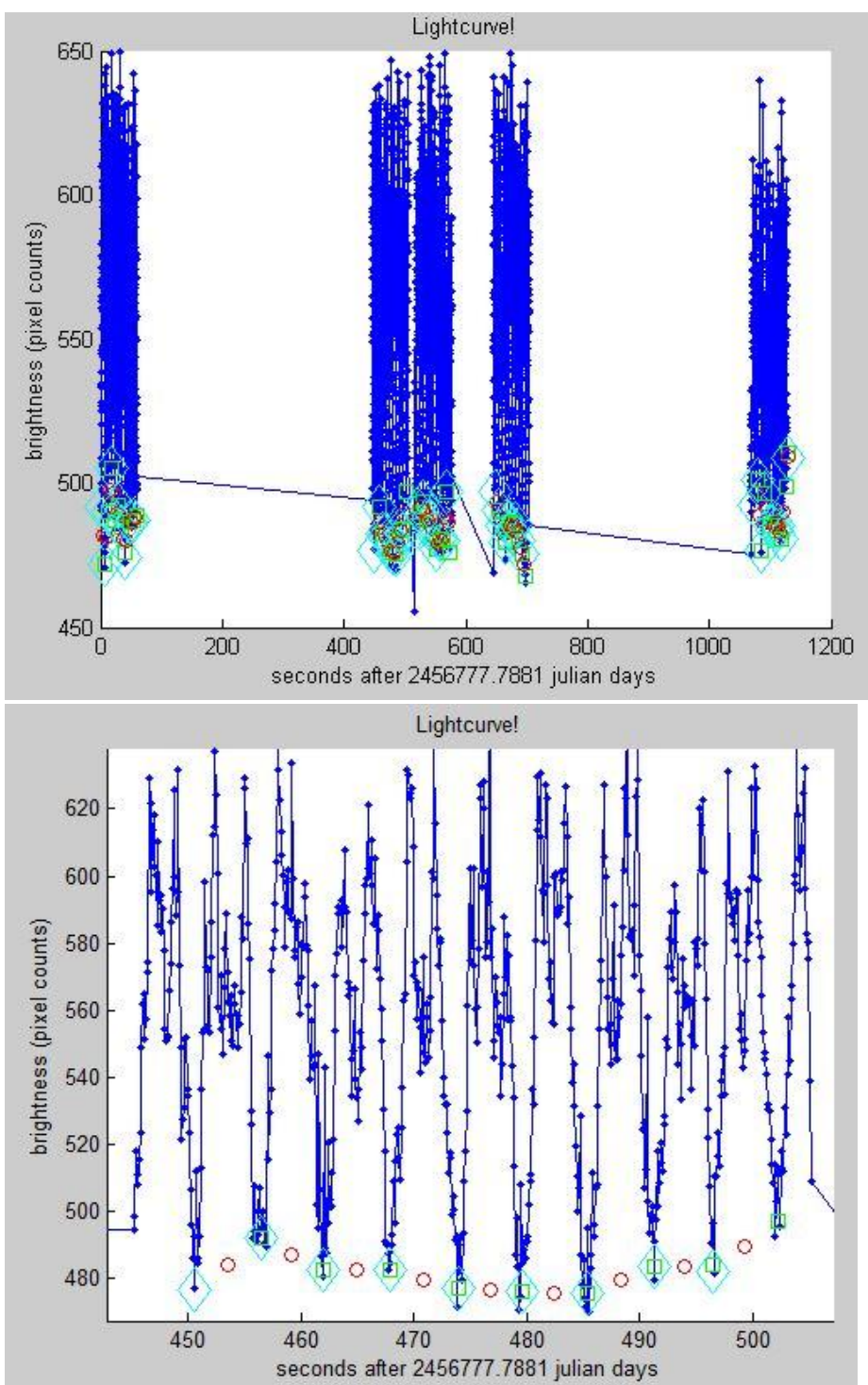

Figure 25. Example Light Curves for Obtaining Flash Times. The left figure is the light curve for one object, where five different images were taken in about 1200 seconds. This is after the flash times have been obtained because the markers that are plotted are visible. The right figure is a zoomed in version of the same light curve but for only the second image that was taken in this set.

simply helped to identify where the last click was made when scrolling across to see the next section of the light curve. The coordinates of those two points are averaged to plot a red circle, which marks the time at 
which the flash is estimated to have occurred at. The "y" value of the red circle is unimportant, as only the time of the flash, and not the brightness of the flash, is important. If a part of the data included a particularly large amount of noise, and the periodic behavior could not be observed, then no marks were placed along that portion of the light curve. Similarly, if a bright star appears in the middle of a streak, then that portion of the light curve was not used. If a mistake is made, then the time of the misplaced red circle is noted, and can all mistakes can be deleted later in the code before further analysis is done.

At the end of the light curve for the night, a mark is placed at a time greater than the end time of the last picture to signal that the end has been reached and this point is not counted. Mistake points can then be deleted, after which the time between each flash is then plotted, and the average time between flashes is calculated. However, this is very likely to be the incorrect flash period because if any periods were skipped over because the data was noisy, then the time between one flash and the next is much larger than expected. Also, as the time that was not recorded in an image produces a time between flashes of at least a minute because it would take about that long to slew the telescope to a new position before the next picture was taken. Therefore, the user is asked to look at the plot that was just created, and estimate the flash period by looking for where the most points are located. Points predicting a flash period with times above a certain value (usually a number between the estimated flash period and twice the flash period) are then deleted, and the updated average flash period is then calculated. This is the value used for the synodic flash period, and the error used is twice the standard deviation of the remaining points under the threshold. An example of this is demonstrated in Figure 26. Finally, the flash times (in units of seconds past the first flash), the Julian date corresponding to the first flash, and the flash average are saved to be used in the final step of data analysis.

\section{Determination of Sidereal Period and Spin Axis Orientation}

The final step of data analysis finds the best candidate spin axis vector and sidereal period. There were several issues with coding it as described in the introductory section, which included units not matching, and the fact that there is no difference between a clockwise rotation and a counterclockwise rotation. This is why two possible spin axis vectors would come up as good solutions. To solve both of these issues, a new method was developed that deals with the issue of the remainder, and takes into account the direction 
of rotation. As a result, only one possible spin axis vector is found as the best possible candidate direction instead of two.
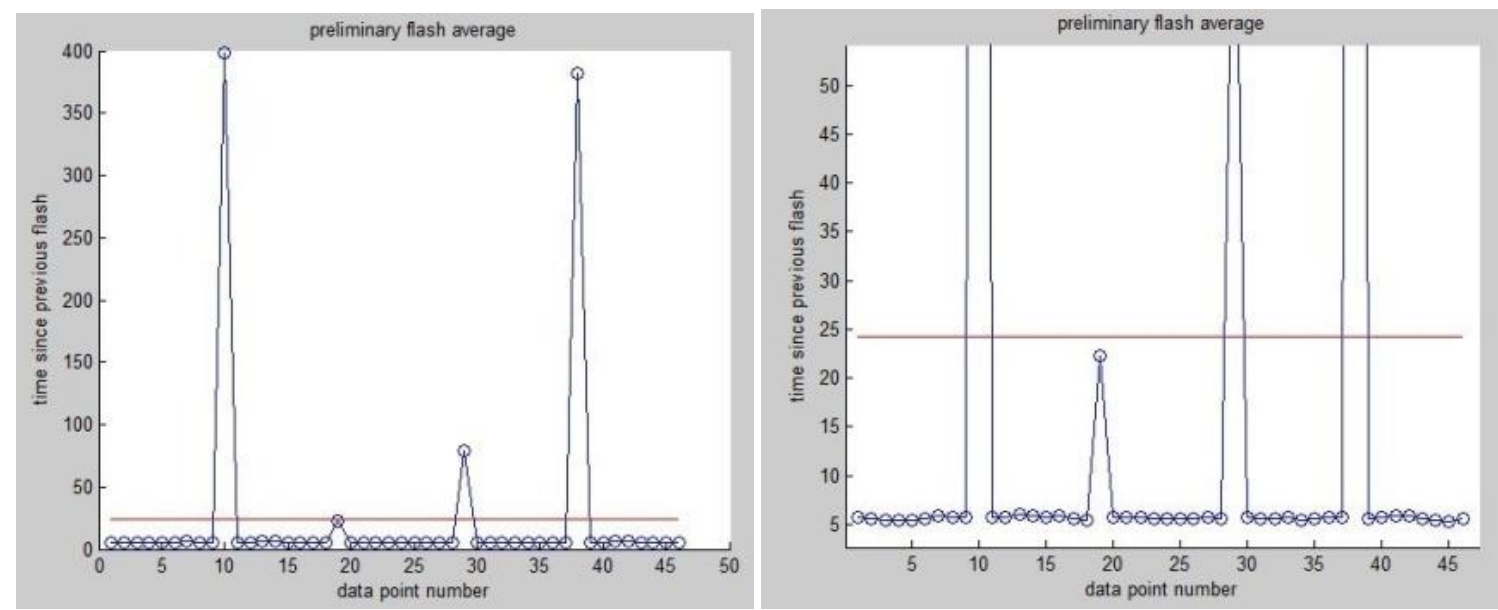

Figure 26. Estimation of the Average Flash Period. The top left plot shows the corresponding data points for the light curve presented in Figure 25. The blue circles are the time between flashes, so there is one less than the number of flashes that were recorded. The red line is the estimated flash period. As can be seen, the time from the last flash in one image to the first flash in the next image is rather large compared to the actual flash period, and throws off the average. The top right figure is a zoomed in version of the first plot, and shows that a threshold between 10 and 20 seconds would be a good choice. After the data points above that threshold are removed, the

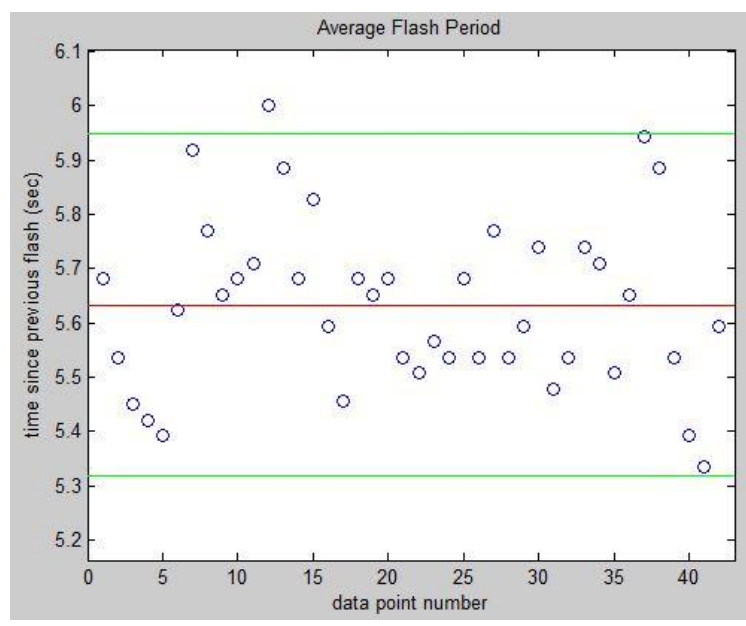
data is shown in the bottom figure, with the new best flash period estimated in red. Twice the standard deviation from the average flash period is shown by the green lines. Note that all flashes are saved for later use, deleting points from this graph only serves to calculate the average flash period.

The method used for data reduction is almost the same as the Somers ${ }^{[1]]}$ method introduced in the beginning, but differs only slightly in the calculation of the Angle A and Angle B for use in calculating the score values. Recall that Angle A was defined as the difference between the body axis vector at the time of the first flash, and where the body axis is for the current flash. Angle B was the theoretical angle (measured from the reference body axis) that should describe the location of the current body axis vector if the guessed sidereal period and spin axis vector are correct. This angle was based on the amount of time that had passed since the first flash. Subtracting Angle A and Angle B will give the difference between the 
theoretical and experimental values of the body axis vector, however, this does not take into account the fact that one angle could be measured counterclockwise and the other measured clockwise! This makes a difference if the angles are $5^{\circ}$ clockwise and $5^{\circ}$ counterclockwise (just theoretical example numbers). The score for this position would be zero even though it should be 10. To fix this issue, the experimental position of the body axis vector was calculated and labeled Body A, (which is the analog of Angle A), and the theoretical position of the body axis vector was also calculated and called Body B (which is the analog of Angle B). Then, one can directly find the angle between these two vectors that would return the value of 10 in that theoretical example.

To find Body A: the same technique was used of crossing the phase angle bisector with the spin axis, however, if that cross product is done in the same way each time, the body axis will line up in the same relative direction as it was at the time of the first flash. To take into account that flashes occur twice per sidereal rotation period, the body axis vector must alternate between approximately $0^{\circ}$ and $180^{\circ}$ from the reference body axis vector. To figure out the direction, the time that had passed since the reference time was divided by the flash period, and this quotient rounded to the nearest whole number. If an even number of flash periods had passed, then the body axis vector was calculated by crossing the spin axis vector and the current phase angle bisector in the same order as had been done for the reference flash. Else, if an odd number of flash periods had gone by, then the spin axis vector and the current phase angle bisector were crossed in the opposite order as for the reference body axis, therefore obtaining the body axis pointing in the opposite direction. A visualization is shown in Figure 27.

Using the assumption that the spin axis vector is constant and that the body axis vector is perpendicular to the spin axis vector, Body B was calculated. This yields the result that the body axis vector is always contained in the plane perpendicular to the spin axis vector. Within this plane, the original body axis vector is defined as the "x" direction, which has a angle value of $0^{\circ}$ on a unit circle. The "y" direction was found by crossing the spin axis vector with the $\mathrm{x}$ direction that makes a right handed coordinate system, and defines the target to be spinning counterclockwise when viewed from above. Any vector in this $2 \mathrm{D}$ plane can be described by some multiple of the $\mathrm{x}$ and $\mathrm{y}$ direction vectors. The equation to describe the theoretical body axis vector at any point in time is given by Eqn. (2). 


$$
\begin{gathered}
\text { body axis vector }=\cos (\theta) * \mathrm{~B}_{0}+\sin (\theta) * \mathrm{~B}_{\perp} \\
\qquad \theta=\frac{2 \pi *\left(t-t_{0}\right)}{\text { sidereal period }}
\end{gathered}
$$

In Eqn. (2), $B_{0}$ is the reference body axis vector, $B_{\perp}$ is the "y" direction which is perpendicular to the spin axis vector and the reference body axis vector. The angle $\theta$ is the angle rotated by the object since the first reference flash, and is given by the time difference since the reference flash divided by the sidereal flash period and multiplied by $2 \pi$. This ensures that the object rotates following the right handed coordinate system. An illustration is shown in Figure 28.

Once both the Body A and Body B vectors have been found, the angle between them is easily calculated using the arccosine of the dot product, and that angle is used as the score for that flash. The scores for each flash are added to form the total score for that RA, DEC, and spin axis vector combination just as they were in the method presented by Somers ${ }^{[11]}$.

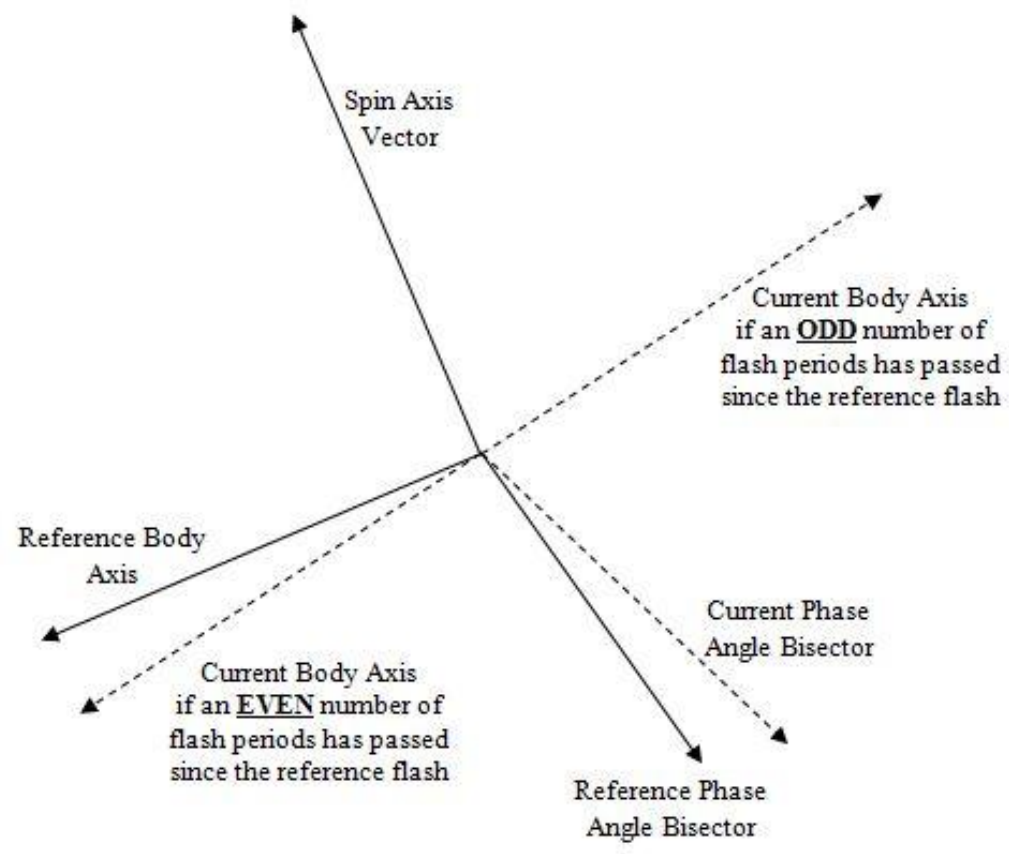

Figure 27. Illustration of the Body A Vector. The reference body axis is perpendicular to the reference phase angle bisector and spin axis vector. Both possibilities for the current body axis are perpendicular to the current phase angle bisector and the spin axis vector. The phase angle bisectors are not necessarily perpendicular to the spin axis vector. 


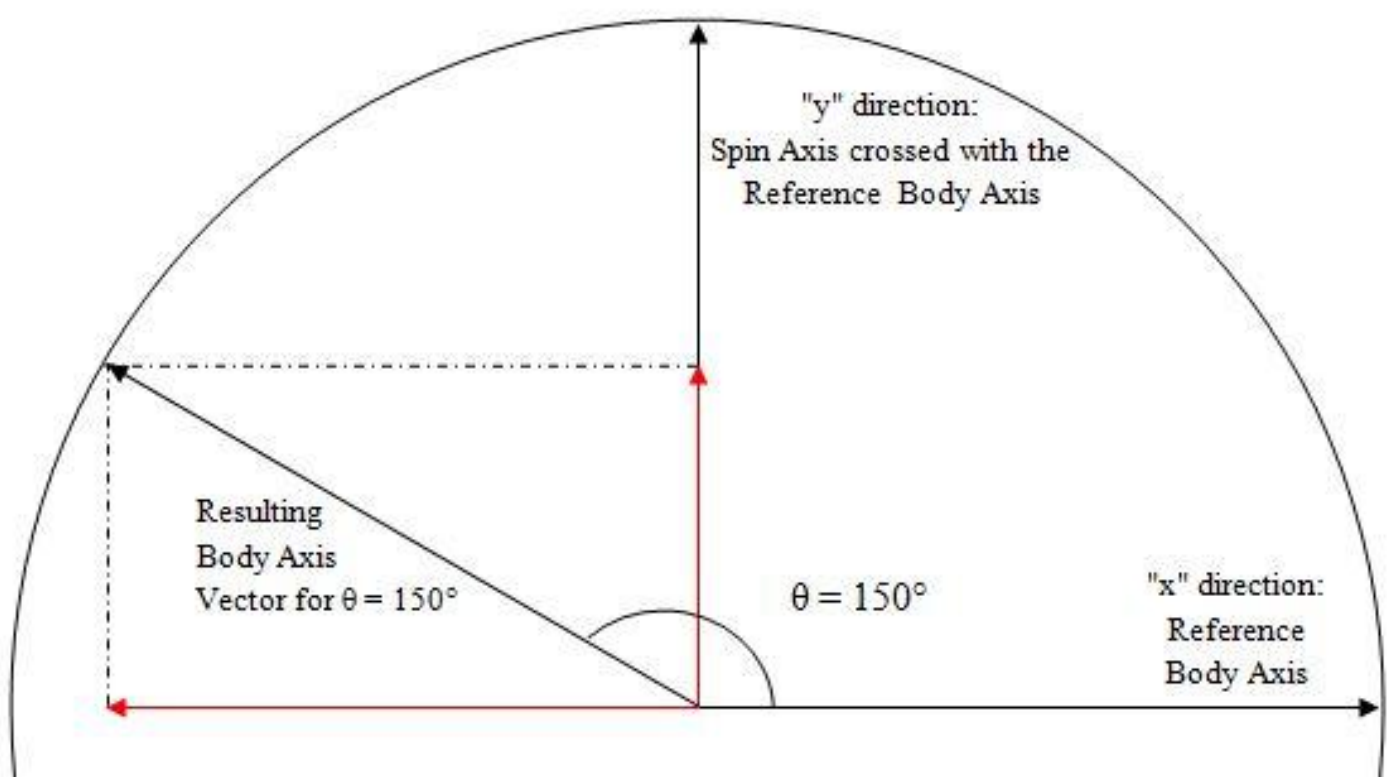

Figure 28. Illustration of the Body B Vector. The reference body axis is the $x$ axis and the spin axis (coming out of the page) crossed with the reference body axis defines the y direction. The red vectors are the components of the new body axis vector in terms of the original $x$ and $y$ directions. 


\section{RESULTS AND DISCUSSION}

In this section, the test cases created to validate the code will be discussed first before the actual observations are discussed.

\section{A. Test Cases for Validation}

Three test cases were created to validate the method for determining the spin axis orientation and sidereal period. All three were based on the target SSN 37811 and is for the night of May $13^{\text {th }} 2014$ and flashes were predicted from 4 am to 8 am UTC (May 12 at 9 pm to May 13 at 1 am local time). The flashes were simulated for the Cal Poly Observatory: a latitude of $35.3^{\circ} \mathrm{N}$, a longitude of $120^{\circ} \mathrm{W}$, and an altitude of 105 meters. All test cases were made with a sidereal rotational period of 5.4 seconds, and the RA,DEC choices for the three cases (in order) are $(50,70),(150,-50)$, and $(300,15)$. After the test case is run through the sidereal period and spin axis calculator program a 3D matrix of scores is produced, and a contour plot is made for the constant sidereal period level with the lowest score. The contour plots for these three test cases can be seen in Figure 29.

If all four hours of exact flash times are entered into the program with the exact flash period, then the simulation produces the correct RA and DEC values. This seems trivial, as it should work, but the predicted flash times are so close together for the three cases that it the program needs two and a half to three hours of flash data before it can correctly predict the RA and DEC values. This is because the phase angle bisector changes very slowly for objects near the GEO regime. That being said, the program obviously did not produce correct results if only twenty minutes of data was used. For this simulation, the twenty minutes of data was spaced as if ten one-minute exposures were taken with a minute in between each exposure at the start of the flash window, and the other ten one-minute images were similarly spaced, but were taken two hours after the first image. 


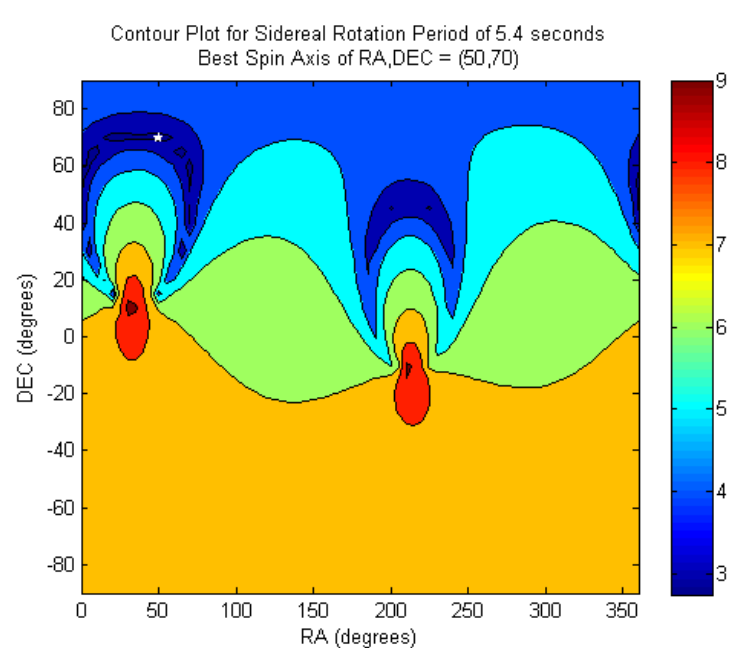

Contour Plot for Sidereal Rotation Period of 5.4 seconds Best Spin Axis of RA,DEC $=(300,15)$

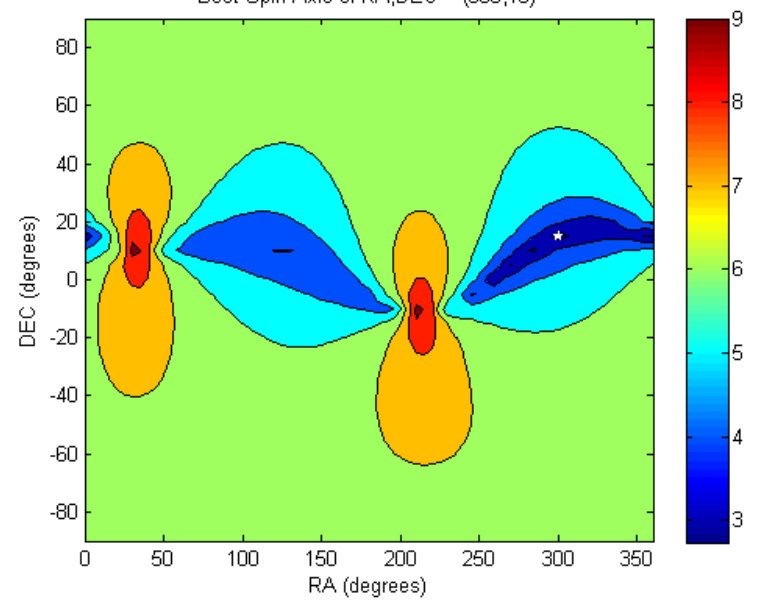

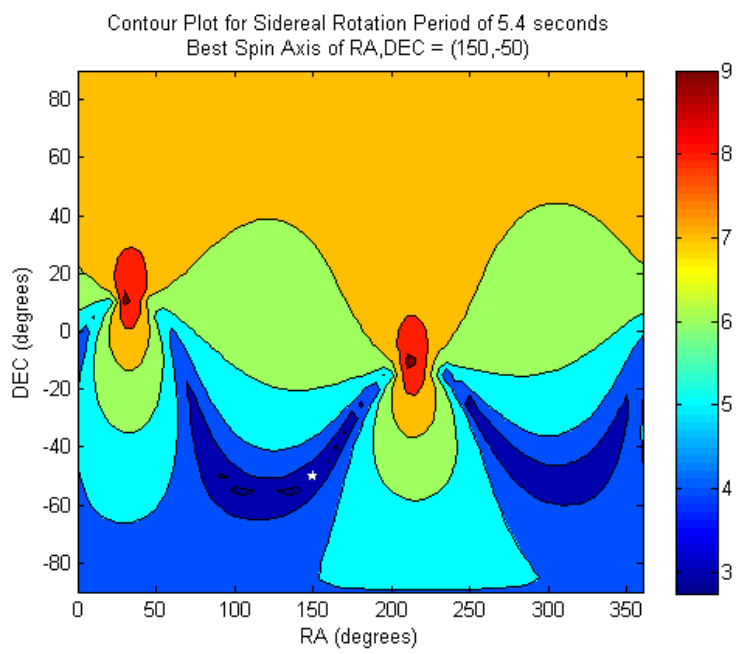

Figure 29. Contour Plots for Test Data. The three cases are shown in order from right to left, top to bottom. The white star in the image shows the location of the RA and DEC combination with the lowest score. In all three test cases, the correct spin axis direction was found using all four hours of perfect simulated flash times. Because the scores cover such a large range of numbers, the natural log of the score is shown so that different contour levels can actually be seen.

Since the code showed no hope of obtaining the correct spin axis vector with limited data from only one night (especially considering simulated data is perfect, while collected data is not), a second set of test cases was created. This second set contains three cases just as before with the same RA and DEC values listed above. The second set was for the night of May $16^{\text {th }} 2014$ from 4 am to 7 am UTC (May 15 at 9 pm to May 16 at midnight local time). When all three hours of perfect flashes are used, the right answer is the direct result (remembering that taking into account the direction of the spin eliminates the wrong axis). Once again, when just the twenty minute subset of data was taken, the results were less than ideal. It was hoped that if the subsets of data from the two separate nights were reduced together then the result would get closer to the exact solution, however, that was not the case. The results of these subset test cases are shown in Table 3 and the contour plots for the combined night subset tests are in Figure 30. 

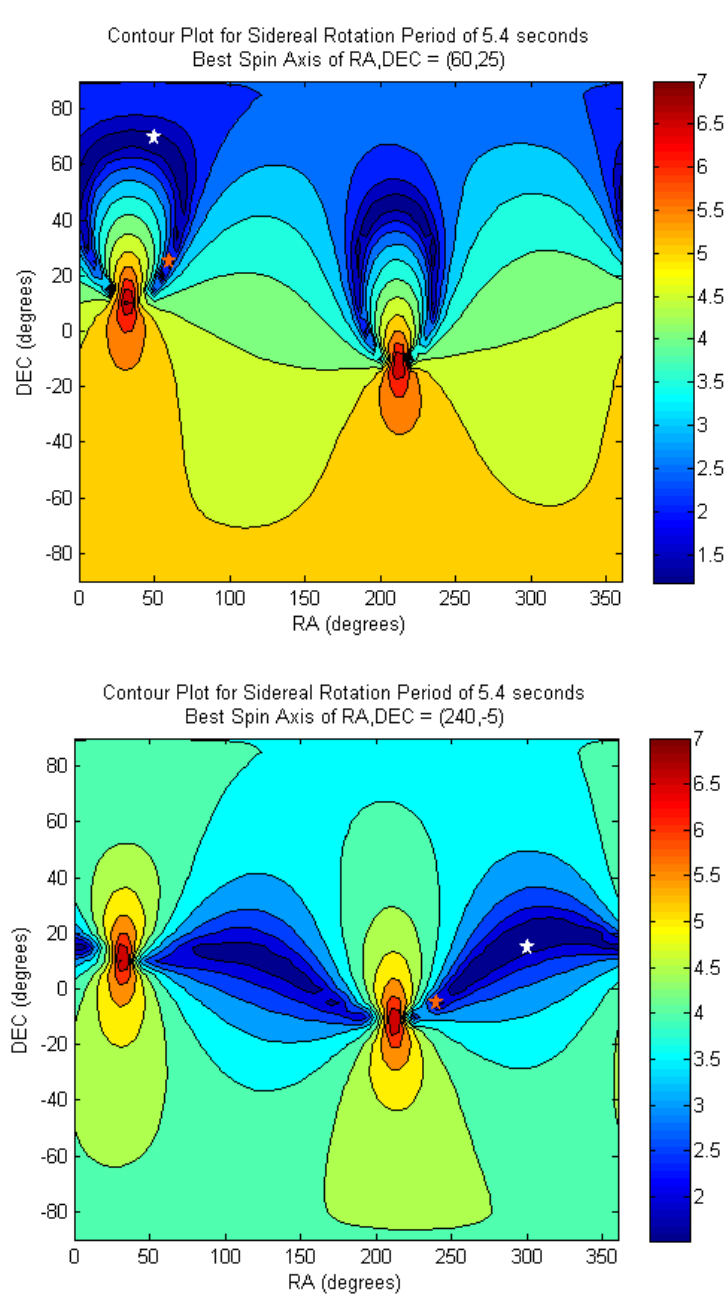

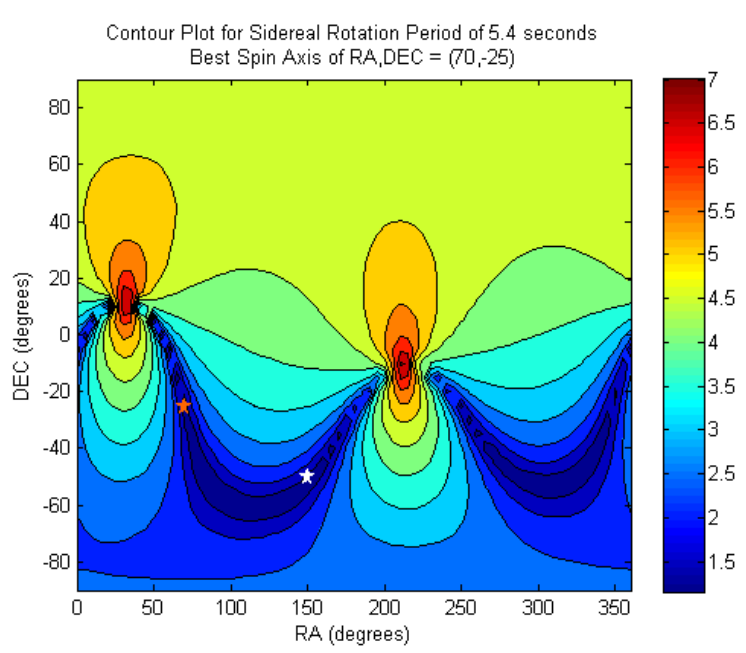

Figure 30. Contour Plots for Subset of Test Data. The twenty-minute subsets of test data from both nights were combined for each test case. The three test cases are shown in order: top left, top right, and bottom left. White stars are the correct $R A$ and DEC while orange stars are the program results. The natural log of the scores is shown so all contour levels are visible. In all three test cases, the plots look similar to the plots that converged to the right answer, however, the spin axis that got the lowest score was not the correct answer. Note that direct comparison of the score magnitude between these plots and the previous plots is not possible because the score changes drastically if more or less flashes are tested.

Table 3. Test Case Results

\begin{tabular}{|c|c|c|c|c|c|c|c|}
\cline { 3 - 7 } \multicolumn{2}{c|}{} & \multicolumn{3}{c|}{ Program Outputs } & \multicolumn{2}{c|}{ Errors } \\
\hline \multirow{2}{*}{ Test Case } & $\begin{array}{c}\text { Correct } \\
\text { RA/DEC }\end{array}$ & Night & $\begin{array}{c}\text { Sidereal } \\
\text { Period (s) }\end{array}$ & $\begin{array}{c}\text { RA } \\
(\mathrm{deg})\end{array}$ & $\begin{array}{c}\text { DEC } \\
(\mathrm{deg})\end{array}$ & $\begin{array}{c}\text { RA error } \\
(\mathrm{deg})\end{array}$ & $\begin{array}{c}\text { DEC error } \\
(\mathrm{deg})\end{array}$ \\
\hline \multirow{3}{*}{$\mathrm{A}$} & May 12 & 5.4 & 70 & 45 & 20 & 25 \\
\cline { 3 - 8 } & \multirow{3}{*}{$(50,70)$} & May 15 & 5.4 & 25 & 70 & 25 & 0 \\
\cline { 3 - 8 } & & Combined & 5.4 & 60 & 25 & 10 & 45 \\
\hline \multirow{3}{*}{$\mathrm{B}$} & \multirow{3}{*}{$(150,-50)$} & May 12 & 5.4 & 270 & -40 & 120 & 10 \\
\cline { 3 - 8 } & & May 15 & 5.4 & 80 & -20 & 70 & 30 \\
\cline { 3 - 8 } & Combined & 5.4 & 70 & -25 & 80 & 25 \\
\hline \multirow{3}{*}{$\mathrm{C}$} & \multirow{3}{*}{$(300,15)$} & May 12 & 5.4 & 255 & 0 & 45 & 15 \\
\cline { 3 - 8 } & & May 15 & 5.4 & 265 & 0 & 35 & 15 \\
\cline { 3 - 8 } & & Combined & 5.4 & 240 & -5 & 60 & 20 \\
\hline
\end{tabular}

For all cases, the exact sidereal period was found correctly, but the same cannot be said for the spin axis vectors. The average RA error is $52^{\circ}$ and the average DEC error is $20^{\circ}$. Therefore, since this 
simulation was done for 40 images of perfect data, and experimental data is not perfect, even if more than 40 images were collected for one object the results for the spin axis vector would likely still be very wrong, or at best, have very large error bars. An important thing to note, however, is that the result spin axis vector appears in the same contour line as the correct spin axis vector for all of the test cases presented in Table 3 which is easily seen in Figure 30. The only exception to this is Test B for the night of May $12^{\text {th }}$ which is the test case with the largest RA error. This large RA error (but low DEC error) occurs because the resulting spin axis vector appears in the low scoring contour region that is not the correct low scoring contour region, and the two best contour regions are approximately opposite in RA but relatively similar in DEC. The contour plot for this specific case is shown in Figure 31.

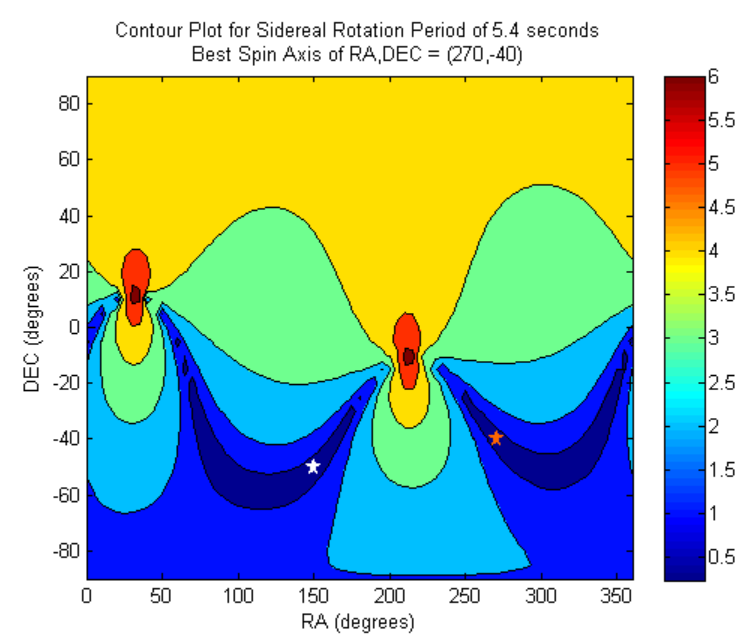

Figure 31. Test Case Contour Result for Test B on May 12. Of all the test case data subsets created, this was the only case where the resulting spin axis was not in the same low scoring contour region as the correct spin axis. The white star is the correct spin axis while the orange star is the resulting spin axis. Because the two low scoring contour regions are opposite each other in RA but approximately the same in DEC, this test case had a relatively large error in $R A$ but still had a relatively low error in DEC. It is still important to note that the lowest scoring contour region as a whole does indeed contain the correct spin axis.

While the spin axis direction cannot be accurately determined, it was noticed that each trial case returned the exact sidereal period. The best score for all the RA, DEC, and sidereal period combinations was found on the level of the 3D matrix for the correct sidereal period. It was also noticed that if all the scores for all RA/DEC combinations are added for a given sidereal period, a score for the sidereal period can be generated. The importance of the score for the sidereal period is that when the correct sidereal period is tested, all of the RA/DEC combinations tend to have slightly lower scores. This trend is shown in Figure 32 for one of the cases above, but is true of each test case that was run. The approach of finding the RA/DEC level with the lowest total score was then applied to the observational data to help in the determination of the best sidereal period. 


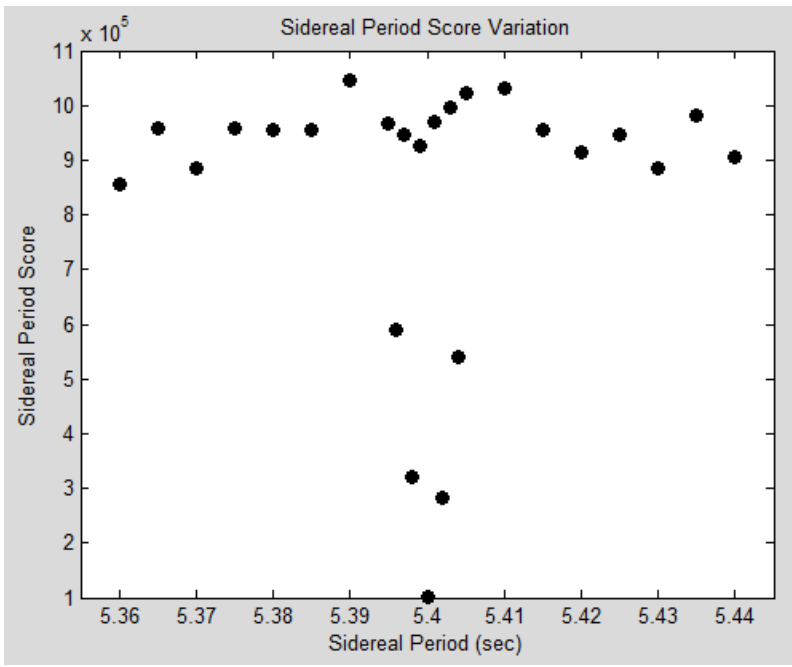

Figure 32. Sidereal Period Score vs. Sidereal Period. The best score is clearly at 5.4 seconds, which is indeed the correct sidereal period.

\section{B. Observational Results}

A list of the objects successfully observed is listed in Table 4 with the estimated synodic period for each night, and the best candidate sidereal period. The dates listed are the Pacific Standard Time date on which the sun set thus beginning that night of observations, even if observations continued past midnight. The synodic period error is twice the standard deviation of the time between flashes (see Figure 26), and in general, the standard deviation for the synodic flash period increases with increasing rotation period. The errors for the sidereal period can be no better than the errors for the synodic period, and the combined night errors are the inverse square root of the sum of inverse squares of the errors for each individual night. Following this table is section for each object and a discussion of the observations. As was mentioned, in the previous section, the error values on the spin axis vector would be too large to actually specify a value for the spin axis. Viewing the contour plots may give some insight into the correct spin axis, and all contour plots for the observational data results are shown in APPENDIX F: Contour Plot Results for Observational Data.

\section{Target 36034}

Target 36034 had the smallest standard deviation error, which is likely because the period of rotation was the fastest for this object compared to the other targets. The possible sidereal rotation periods were 
tested from 1.885 to 1.945 in steps of 0.001 because the rotational period is so short. In addition, there were many images of this target, (compared to some of the other targets that did not have as much data) which increases confidence in the calculated sidereal period value. The sidereal period plot for this target is shown in Figure 33 although it was not particularly enlightening because the scores were all very close.

Table 4. Best Candidate Synodic and Sidereal Periods

\begin{tabular}{|c|c|c|c|c|}
\hline $\begin{array}{c}\text { Target } \\
\text { SSN }\end{array}$ & Date & $\begin{array}{c}\text { Images } \\
\text { Used }\end{array}$ & $\begin{array}{c}\text { Synodic Period } \\
(\mathrm{sec})\end{array}$ & $\begin{array}{c}\text { Sidereal Period } \\
(\mathrm{sec})\end{array}$ \\
\hline \multirow{3}{*}{36034} & April 28 & $10+10$ & $1.9 \pm 0.2$ & $1.9 \pm 0.2$ \\
\cline { 2 - 5 } & May 1 & $10+5$ & $1.9 \pm 0.2$ & $1.9 \pm 0.2$ \\
\cline { 2 - 5 } & Combined & NA & NA & $1.9 \pm 0.1$ \\
\hline \multirow{3}{*}{37777} & April 26 & 5 & $3.6 \pm 0.6$ & $3.7 \pm 0.6$ \\
\cline { 2 - 5 } & April 29 & 9 & $3.7 \pm 0.4$ & $3.7 \pm 0.4$ \\
\cline { 2 - 5 } & Combined & NA & NA & $3.7 \pm 0.3$ \\
\hline \multirow{3}{*}{37811} & April 23 & 10 & $5.4 \pm 0.6$ & $5.4 \pm 0.6$ \\
\cline { 2 - 5 } & April 28 & $10+10$ & $5.4 \pm 0.4$ & $5.4 \pm 0.4$ \\
\cline { 2 - 5 } & May 1 & $9+10$ & $5.4 \pm 0.4$ & $5.4 \pm 0.4$ \\
\cline { 2 - 5 } & Combined & NA & NA & $5.4 \pm 0.3$ \\
\hline \multirow{3}{*}{39218} & April 23 & 13 & $11.3 \pm 0.8$ & $11.2 \pm 0.8$ \\
\cline { 2 - 5 } & April 26 & 10 & $11.3 \pm 0.6$ & $11.2 \pm 0.6$ \\
\cline { 2 - 5 } & April 29 & 5 & $11.3 \pm 0.6$ & $11.2 \pm 0.6$ \\
\cline { 2 - 5 } & Combined & NA & NA & $11.2 \pm 0.4$ \\
\hline 39235 & April 28 & $10+9$ & $3.6 \pm 0.4$ & $3.6 \pm 0.4$ \\
\hline
\end{tabular}

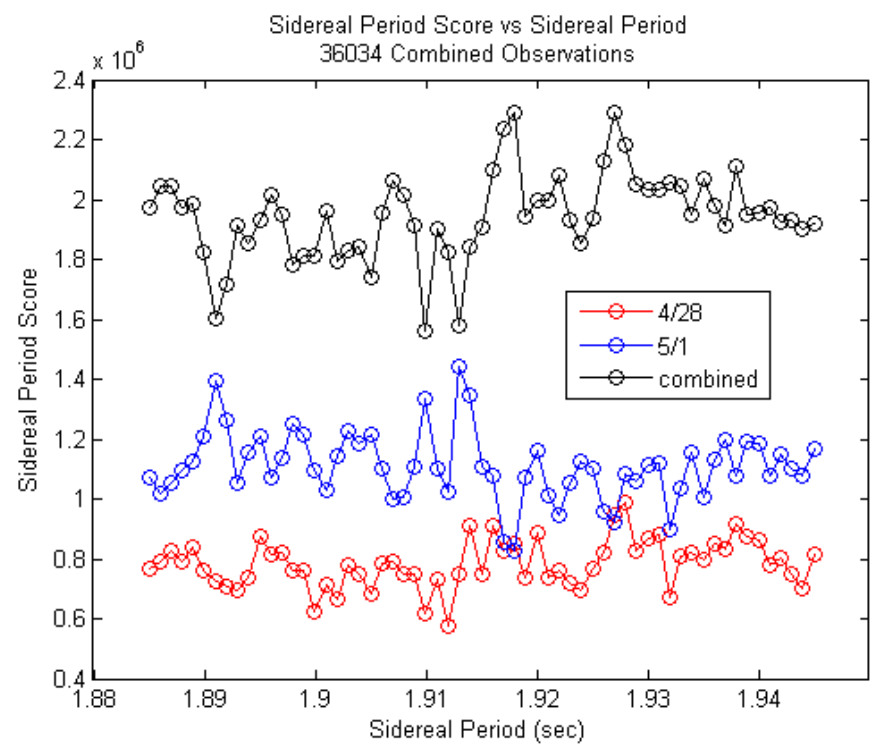

Figure 33. Sidereal Period Score for Target 36034. The scores for this target were much closer than for the test data but minimums can still be seen near 1.91 seconds for the data from April 28 and the combined night data. A minimum can be seen in the May 1 data closer to 1.92 seconds. 


\section{Target 37777}

This target was captured on two separate nights, however, has the least amount of data. The first night only half of the images were kept which explains the higher standard deviation for the first night. The second night, another single set of ten images were taken, but nine were kept, thus the lower standard deviation. Sidereal periods were tested in steps of 0.005 seconds from 3.557 to 3.702 seconds. The sidereal period score graph for this object was rather interesting, in that it has two distinct local minima that are in the exact same location for both the individual night reductions as well as the combined night observation. This can be seen in Figure 34. The best RA, DEC, and sidereal period in each case corresponded to the sidereal period shown above of 3.647 seconds, and the other minima was located at a sidereal period of 3.592 seconds.

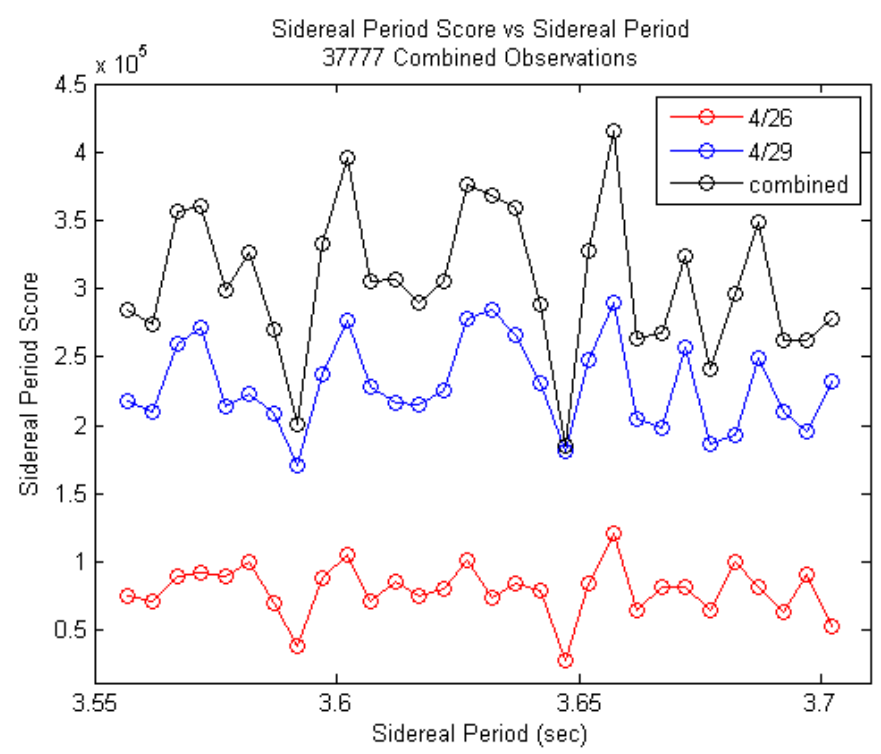

Figure 34. Sidereal Period Score for Target 37777. The two local minima can be seen to overlap for the combined data and for the data taken on April $29^{\text {th }}$. The data taken on April $26^{\text {th }}$ has a lower score overall, but the two local minima can be seen to be at the exact same sidereal periods. 


\section{Target 37811}

Target 37811 was captured on three different nights. The first night (April 23) did not have the best seeing conditions, and therefore has a higher standard deviation of flash periods. The second and third captured two sets of ten images separated by over two hours. The second two nights of observations of this object had much better sky conditions, and as a result the standard deviation of the flash period is lower.

This object has the most amount of usable images of all the targets captured, however, it does not have a clear minimum sidereal period when looking at the sidereal period score plot, except for the second night of observations and the combined analysis. The sidereal period score plot for this target is shown in Figure 35. The first night had the lowest estimated sidereal period, however this is also the night with the worst weather conditions and the least amount of data, hence the higher error value. The second and third nights of data reduction had almost twice the number of images, and the weather conditions were much better. Because the rest of the data analysis for the second two nights shows the same values it would seem that 5.38 seconds is the best estimated sidereal rotation period for this object.

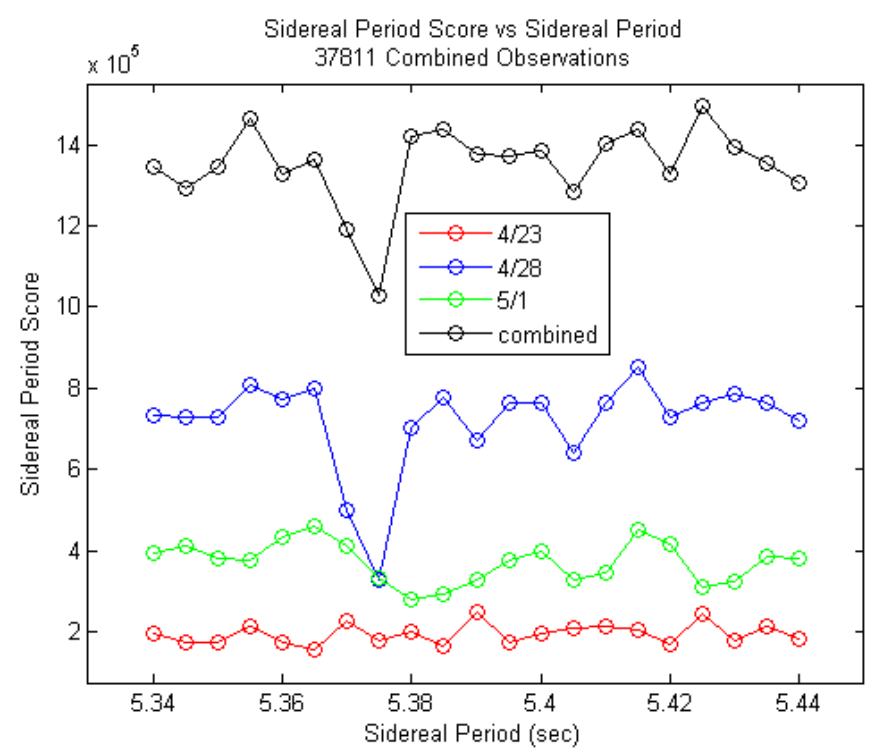

Figure 35. Sidereal Period Score for Target 37811. Sidereal periods of 5.34 to 5.44 were tested in steps of 0.005. The local minima for the April $28^{\text {th }}$ data and the combination data is clearly seen at 5.375 seconds, while the May $1^{\text {st }}$ lowest score is not so easily seen at 5.38 seconds. The data for April $23^{\text {rd }}$ was subject to poor weather conditions, and therefore does not show a strong preference to any particular sidereal rotation period. 


\section{Target 39218}

Target 39218 was the first Ariane 5 target that was observed, and also happened to be a very obvious tumbling rocket body! The first set of data for object 39218 was taken on the same night as the first observations of 37811 , which was the night that did not have good seeing. Therefore, the standard deviation of observed flashes was again higher on this first night then for the following two nights. This also contributed to a higher error for the sidereal period for the first night as well. The sidereal period score plot for this target is shown in Figure 36 and is the best defined because the sidereal period was longer compared to the other observed targets. The four local minima are clearly seen for each data set. For the first night and for the combined data, the minima occur at 11.215 seconds, however, the best RA/DEC/sidereal period score happens to be where the sidereal period is 11.205 . This discrepancy leads to a higher error value for these predicted sidereal periods. The data for the second and third nights of observations of 39218 both show the local minima to be at 11.230 seconds, and despite there being less pictures in this set, the weather conditions were better than for the first night, which shows in the synodic period error. This permits a lower error on the sidereal period for these two nights.

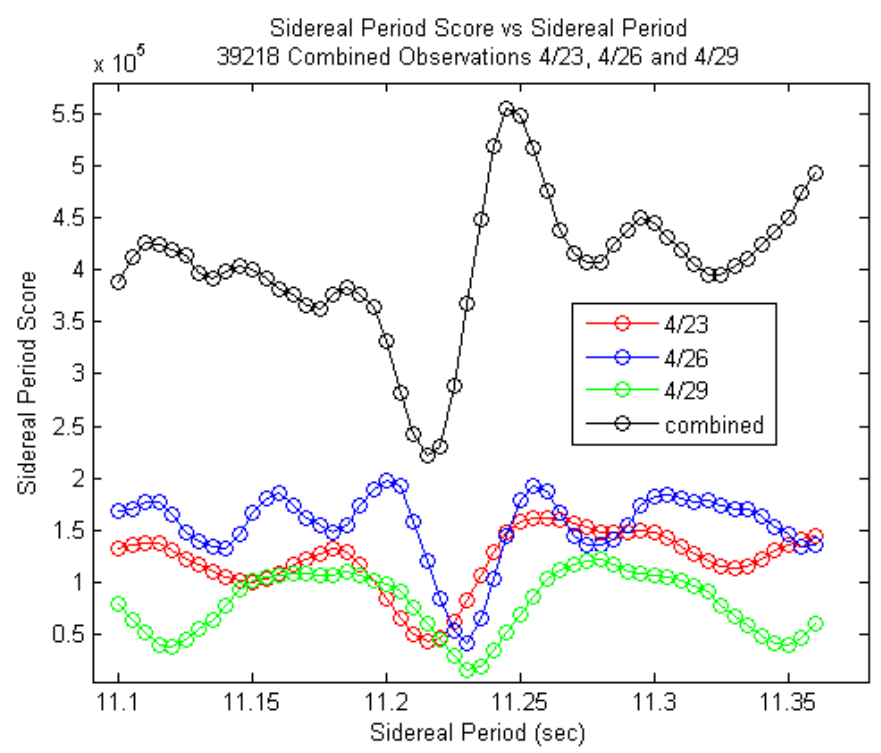

Figure 36. Sidereal Period Score for Target 39218. Data points were tested every 0.005 seconds from 11.100 to 11.360 seconds. The three local minima can be seen in the center of the image between 11.2 and 11.25 seconds for all four data sets. 


\section{Target 39235}

This object was only successfully observed on one night, however, good images were obtained with a relatively low standard deviation error. The sidereal period score was tested in 0.005 second increments from 3.555 seconds to 3.635 seconds, and the sidereal rotation period of 3.6 seconds was found to have the lowest score by a significant margin. A summary of all sidereal period values can be found in Table 4. All other scores tested had a score at least 1.5 times greater except one point at 3.625 seconds that was only 1.3 times greater. This behavior can be seen in Figure 37.

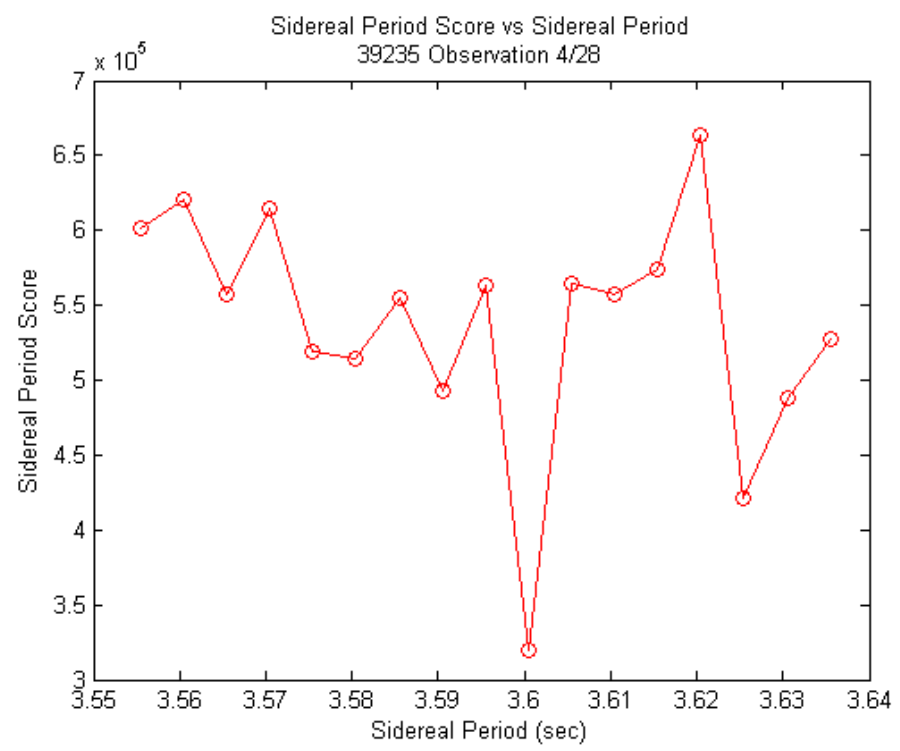

Figure 37. Sidereal Period Score for Target 39235. The local minimum is clearly defined at a sidereal period of 3.60 seconds. 


\section{CONCLUSIONS AND FUTURE WORK}

Ariane 5 rocket bodies were observed over several different nights at the Cal Poly Observatory. Data reduction on all images was performed using IRAF software, and light curves were generated for targets that were bright using self-written Matlab software. For targets whose light curves showed obvious periodic motion, flash times were found and the sidereal spin rate and spin axis were calculated also using verified self-written Matlab software.

Three test cases were created to verify the calculation of the sidereal spin rate and spin axis calculator and all three yielded expected results. However, correct results were only found when enough flash times were provided to distinguish one spin axis orientation from the next. This was on the order of three hours of continuous flash times that had no error. Even when small amounts of test data (similar to what was collected) were combined for separate nights, the errors between the predicted and actual spin axis vectors were still too large to conclude that the spin axis calculation had converged at all for such small data sets.

To determine the spin axis orientation of an object with any reasonable error margin, many more observations will have to be taken of a single object over many nights.

It was noticed in the test cases, however, that the correct sidereal period showed the lowest score over all directions of the spin axis vector, and usually by a significant amount. The technique was used with the observed objects to determine the most likely sidereal rotation period for each of the successfully observed targets. The sidereal periods presented are the best candidate values given the data that has been collected as of yet, but should be taken with the errors presented, as well as the possibility that they could be totally wrong altogether.

Much more data should be collected to continue this research because it is very difficult to determine spin axis orientations and sidereal periods for objects near GEO. This is because the movement of the phase angle bisector is the main geometry needed to distinguish between different spin axis vectors. The epoch method was first applied to LEO satellites where the phase angle bisector changes rapidly for one pass so short observations can be used (on the order of 20 minutes), and multiple passes can be obtained in a single night. However, with GEO objects, the phase angle bisector changes very slowly, meaning much longer 
passes must be observed (on the order of hours), and only one pass is possible per night, meaning several nights of observations are necessary.

More test cases need to be created to test the Epoch method program that determines the spin rate and axis orientation. These new tests will need to have random errors introduced to the predicted flash times to simulate the accuracy of the observational data. The standard deviation of the predicted test flash times should be similar to the standard deviations found in collected observational data. Standard deviation results from observations presented in this paper are shown in Table 4 as half the synodic period errors. Testing the Epoch method program with imperfect flash data would yield a better estimate of how much data needs to be collected on a single object before a solid conclusion on the spin axis direction can be made.

A way to expand this research would be to take short exposures of a target over time as the target is being tracked, which is referred to as Track Rate Mode (TRM) by Somers ${ }^{[12]}$. This would make photometry in IRAF very easy to do for a target (because it was made for stars which are expected to be circular and in roughly the same spot in every image), and would be great for objects that have much longer rotational periods. For example, the sidereal rotational period of target SSN 07382 (as published by Somers ${ }^{[11]}$ ) is 1048 seconds, for which this method would work well. All images used for this paper were taken as long exposures in Sidereal Stare Mode (SSM) as the satellites pass by because the objects observed had rotational periods on the order of seconds. Currently the SkyX software has the ability to set the track rates of the telescope to the rates of an arbitrary target on the screen, but doing this resulted in the telescope rapidly slewing off target in the complete wrong direction. The telescope itself also has the ability to track satellites using the hand paddle controller, but that was also unsuccessful.

Future work specifically related to observing at the Cal Poly Observatory is described in APPENDIX C: Cal Poly Observatory Telescope Set-Up and Shut-Down Procedures. 


\section{BIBLIOGRAPHY}

[1] "Ariane 5." Wikipedia. Wikimedia Foundation, 05 Nov. 2014. Web. 11 May 2014. <http://en.wikipedia.org/wiki/Ariane_5>

[2] "Ariane 5." Arianespace. July 2011. Web. 12 May 2014.

< http://www.arianespace.com/launch-services-ariane5/ariane-5-intro.asp >

[3] "Ccdproc." Space Telescope Science Data Analysis System. NASA and AURA, 15 Oct. 1999. Web. 27 May 2014. <http://stsdas.stsci.edu/cgi-bin/gethelp.cgi?ccdproc.hlp>.

[4] Cowardin, H., Ojakangas, G., Mulrooney, M., Lederer, S., \& Liou, J. (2012). Optical Signature Analysis of Tumbling Rocket Bodies via Laboratory Measurements. Advanced Maui Optical and Space Surveillance Technologies Conference. Kihei, Hawaii.

[5] De Pontieu, Bart. "The Determination of the Rotation Axis of a Tumbling Satellite." Visual Satellite Observer's Home Page. Web. 13 May 2014. <http://www.satobs.org/flash/102/node8.html>.

[6] "Deploying Multiple Satellites with Sylda and Vespa." European Space Agency. European Space Agency, 20 Dec. 2013. Web. 16 May 2014.

<http://www.esa.int/Our_Activities/Launchers/Deploying_multiple_satellites_with_Sylda_and_Vespa>.

[7] "Flatcombine." Space Telescope Science Data Analysis System. NASA and AURA, 4 Dec. 1993. Web. 27 May 2014. <http://stsdas.stsci.edu/cgi-bin/gethelp.cgi?flatcombine.hlp>.

[8] "Orbits and the Ecliptic Plane." Hyperphysics. Georgia State University, Department of Physics and Astronomy. Web. 18 May 2014. <http://hyperphysics.phy-astr.gsu.edu/hbase/eclip.html>.

[9] "Rfits." Space Telescope Science Data Analysis System. NASA and AURA, 7 Jun. 1997. Web. 27 May 2014. <http://stsdas.stsci.edu/cgi-bin/gethelp.cgi?rfits.hlp>.

[10] "Satellite Catalog." Space-Track. Ed. Scitor Corporation. JFCC SPACE/J3, Web. 10 May 2014. $<$ https://www.space-track.org >.

[11] Somers, Phil. (2011). Cylindrical RSO Signatures, Spin Axis Orientation and Rotation Period Determination. Advanced Maui Optical and Space Surveillance Technologies Conference. Wailea, Hawaii.

[12] Somers, Phil. (2012). Cylindrical Satellite Spin Axis Characterisation. Canadian Aeronautics and Space Institute Conference. Canata, Ontario, Canada.

[13] Vallado, David A., and Wayne D. McClain. Fundamentals of Astrodynamics and Applications. 3rd ed. Hawthorne, CA: Microcosm, 2007. Print 
[14] Wallace, B., Somers, P., \& Scott, R. (2010). Determination of spin axis orientation of Geosynchronous objects using space-based sensors: an initial feasibility investigation. Advanced Maui Optical and Space Surveillance Technologies Conference. Wailea, Hawaii.

[15] "Zerocombine." Space Telescope Science Data Analysis System. NASA and AURA, 4 Dec. 1993. Web. 27 May 2014. <http://stsdas.stsci.edu/cgi-bin/gethelp.cgi?zerocombine.hlp>. 


\section{APPENDIX A: Application to LEO Satellites}

Bart De Pontieu describes his method for finding the sidereal rotation period and the spin axis direction very well on the Visual Satellite Observer's Home Page ${ }^{[5]}$ so the details of the method will not be discussed here. This method was replicated with Matlab in the hopes that it would help with validation of the code being used. However, because this method was designed for LEO satellites and does not take into account the assumptions made for GEO objects, it did not converge to the correct spin axis for my test cases, and

my code did not work well on the LEO test case presented by De Pontieu ${ }^{[5]}$. However, it seemed worthwhile to discuss the results found by using De Pontieu's method on the test case he presented of a LEO satellite.

The exact details of his test case are also on the Visual Satellite Observer's Home Page ${ }^{[5]}$ and it consists of two six-minute passes of a specified LEO satellite (SSN 06125). An important thing to note is that there was error introduced into the recorded flash times for both passes. The correct sidereal period is 40 seconds and the correct spin axis has a right ascension of $180^{\circ}$ and a declination of $50^{\circ}$. The contour plot for the first pass can be seen in Figure 38, and the contour plot for the second pass can be seen in Figure 39. Both of the individual passes converge to the wrong spin axis vector, but not the same wrong spin axis vector. Also, both contour plots show a region of low scores where the correct spin axis vector is. When both passes are reduced together, it is similar to just adding the scores for each of the individual passes, and the contour plot is shown in Figure 40. While the correct answer did not have the lowest score for either pass, it has the lowest combined score. However, because error was introduced into the flash times, the final spin axis vector found has a right ascension of $183^{\circ}$ instead of $180^{\circ}$, and a declination of $46^{\circ}$ instead of $50^{\circ}$. The sidereal period calculated is 40.0075 seconds instead of 40 seconds. Given that only about twelve minutes of flash times were given and errors were introduced into those flash times, these are reasonable errors. 


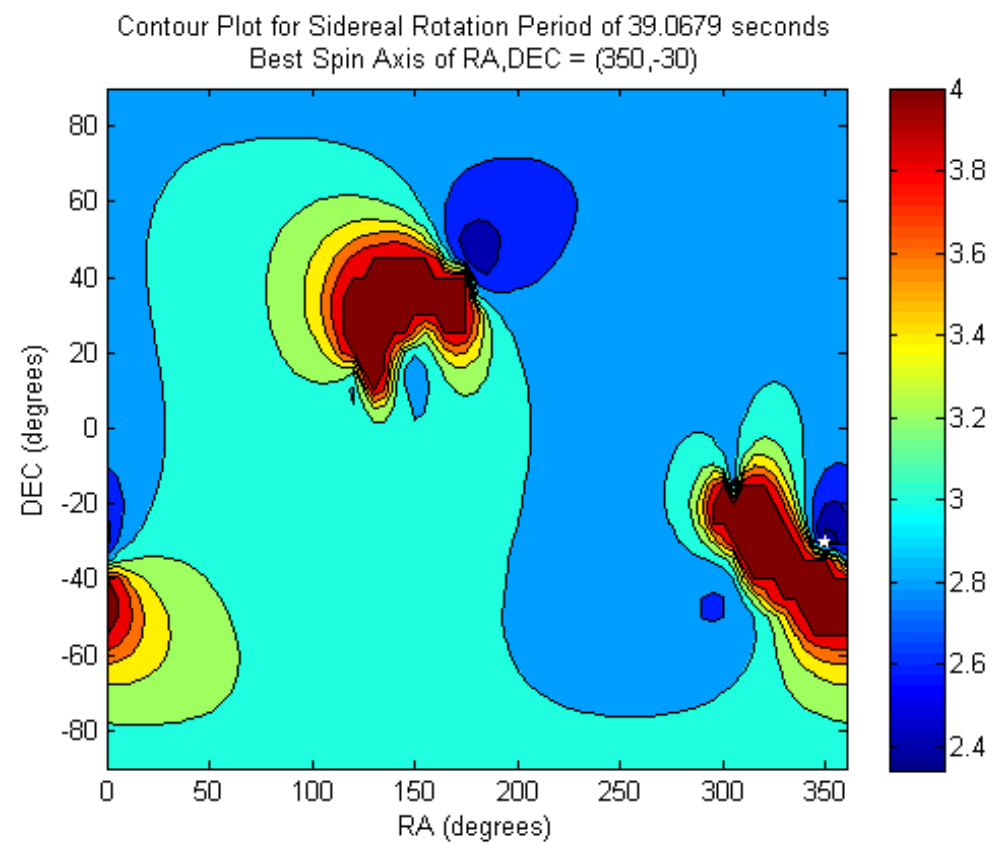

Figure 38. Contour Plot for First Pass of the LEO Test Case Satellite. The most likely spin axis orientation for the first pass has an $R A$ of $350^{\circ}$ and a declination of $-30^{\circ}$ (run in $5^{\circ}$ increments). The region that contains the correct spin axis of 180,50 is also dark blue which means it has a relatively low score, but it did not have the lowest score.

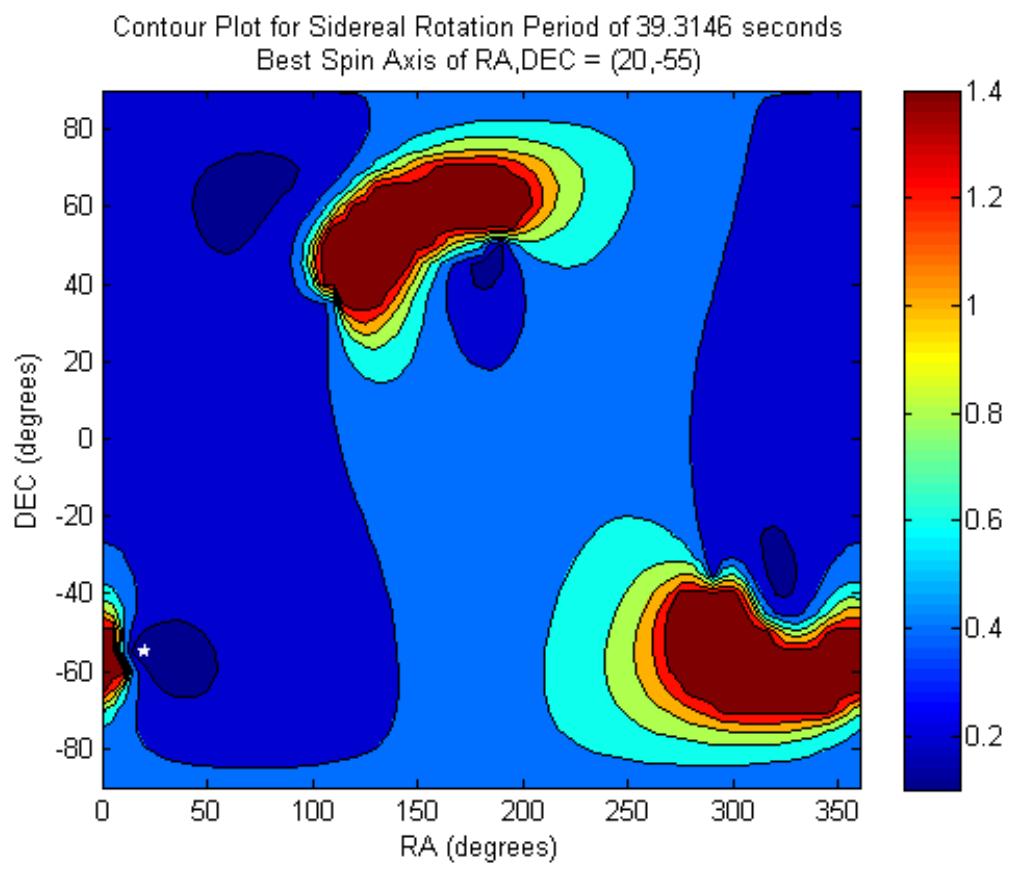

Figure 39. Contour Plot for Second Pass of the LEO Test Case Satellite. The most likely spin axis orientation for the second pass has an RA of $20^{\circ}$ and a declination of $-55^{\circ}$ (run in $5^{\circ}$ increments). The region that contains the correct spin axis of 180,50 is also dark blue which means it has a relatively low score, but it did not have the lowest score. It is important to note that the "wrong" location of the spin axis orientation is in a different region of the sky from where it was in the first pass. 


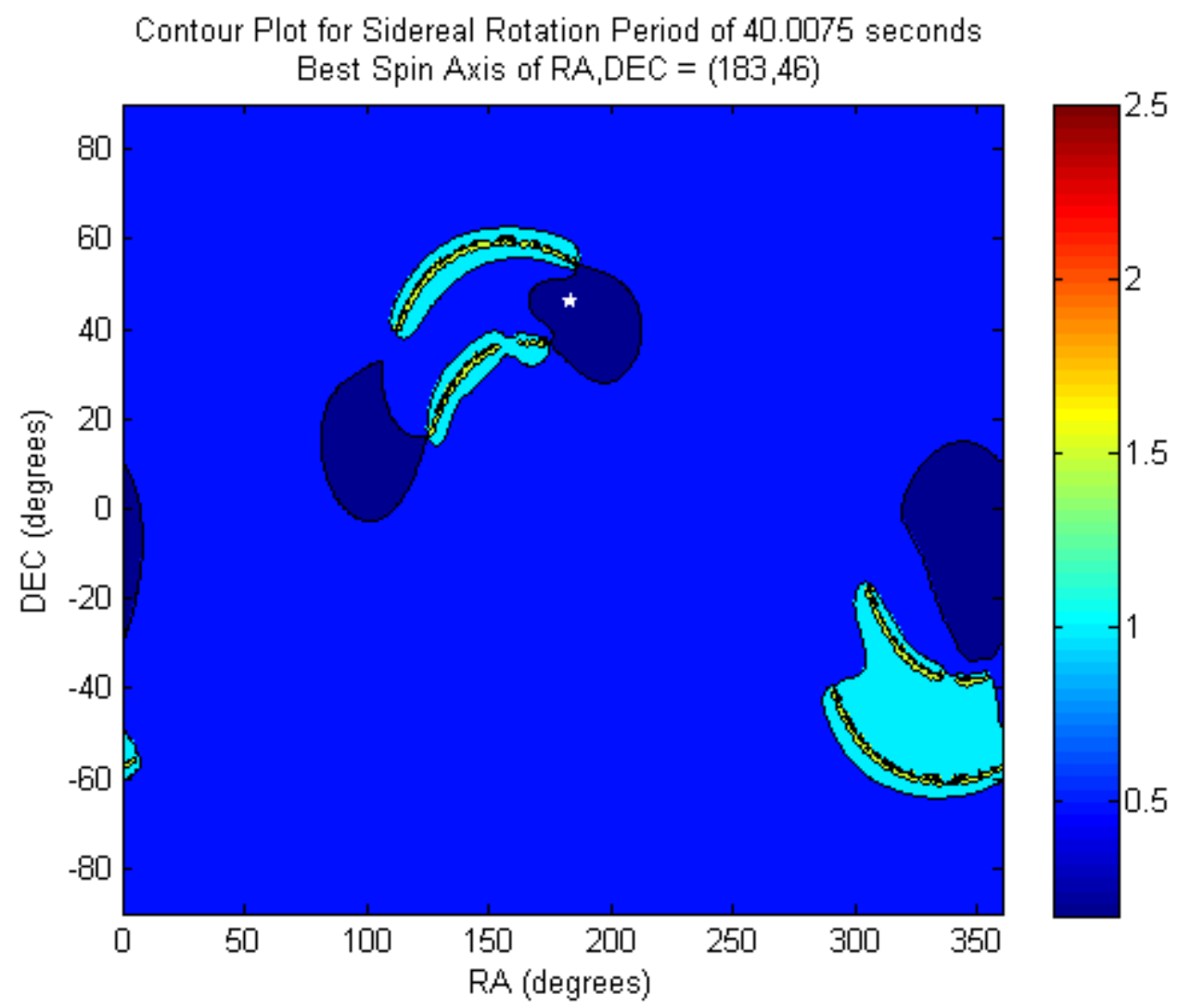

Figure 40. Contour Plot for Both Passes of the LEO Test Case Satellite. The most likely spin axis orientation when both passes are reduced together has a right ascension of $183^{\circ}$ and a declination of $46^{\circ}$ which is close to the exact answer of $180^{\circ}$ and $50^{\circ}$. The sidereal period found for this orientation is 40.0075 seconds while the exact sidereal period is 40 seconds. These errors would seem acceptable given the errors that were introduced and the limited amount of data given in the test case statement. 


\section{APPENDIX B: List of Components and Software Used}

The components used to collect observation images are shown below in Table 5, and the software used for collection or reduction of data are shown below in Table 6 .

Table 5. List of Components used for Observation.

\begin{tabular}{|c|c|c|c|c|}
\hline Component & Brand & Model \# & Serial \# & Notes \\
\hline Telescope & Meade Instruments & $\begin{array}{c}\text { LX600 } \\
\text { Starlock }\end{array}$ & 14" \\
CCD Camera & $\begin{array}{c}\text { SBIG Astronomical } \\
\text { Instruments }\end{array}$ & ST-10XME & 061100506 & $\begin{array}{c}\text { SBIG = Santa Barbara } \\
\text { Instrument Group }\end{array}$ \\
\hline Focuser & $\begin{array}{c}\text { Temperature } \\
\text { Compensating Focuser } \\
\text { (TCF-S) }\end{array}$ & $\begin{array}{c}\text { Celestron } \\
\text { Reducer/Corrector }\end{array}$ & 94175 & US Patent No. 6,327,081 \\
\hline Dome & Digital Dome Works & 4 & 8008 & \\
\hline
\end{tabular}

Table 6. List Software used for Observation or Reduction.

\begin{tabular}{|c|c|c|c|}
\hline Software & Version & Release Date & Notes \\
\hline MATLAB & 7.8 .0 .347 & February 12, 2009 & R2009a Student Version \\
\hline IRAF & Revision 2.16 & May 24, 2012 & Image Reduction and Analysis Facility \\
\hline The SkyX & Professional & 2013 & $\begin{array}{c}\text { Registered to "Cal Poly Physics" } \\
\text { Serial No. D031-1B22618B } \\
\text { Version 10.2.0 (Build 7477) }\end{array}$ \\
\hline CCD Soft & 5.00 .210 & 2011 & Copyright by Software Bisque \\
\hline SAOImage ds9 & 7.2 & April 15, 2013 & \\
\hline
\end{tabular}




\section{APPENDIX C: Cal Poly Observatory Telescope Set-Up and Shut-Down Procedures}

Before anything else can be done, light pollution must be minimized. This is very important because some of the rocket body targets are very dim, and any light pollution can be detrimental to the observation. The Cal Poly campus is well lit at night to give a feeling of safety to its students, so it is impossible to get rid of all light pollution, but there are some steps that can be taken to minimize it. Because the telescope resides in between two buildings, classrooms line either side to the North and South. When classrooms are not in use the lights can be turned off, however, if classrooms are being used then the blinds can be closed. There are also two outside lights that used to be covered with very expensive light covers (cardboard boxes) placed over them by a very complicated mechanism (a two-yard stick) so that this little bit of extra light is minimized, however, there were complaints from the campus police so that is no longer done.

Once the light pollution has been minimized (closing classroom blinds, NOT putting boxes on lights, etc.) the computer in the observatory needs to be turned on. The power strip on the wall needs to be turned on to give power to the dome, and then the dome is opened with the hand controls on the wall of the observatory. If the targets for the night are high in the sky (above $30^{\circ}$ ), then the windbreak on the dome is left down (meaning the windbreak will block the view near the horizon). If the targets are close to the horizon, the windbreak on the dome must be open to allow for these low targets to be visible. If the windbreak is attached to the dome slit before it is opened, then the windbreak will recede upwards with the dome slit when opened, and block high elevation observations and is shown in Figure 9.

By the time the dome is fully open, the computer is done turning on, and is logged into. The camera software as well as the camera are then turned on, so the camera can begin temperature control, and get the CCD chip to a temperature of $-5^{\circ} \mathrm{C}$ which reduces the amount of dark current. The CCDSoft program generates an error that the focuser cannot be connected, but the connection is not necessary to use the focuser. While the CCD is cooling, the telescope can then be uncovered: the giant tarp is removed and the lens caps for both the main telescope and the finder scope are removed. The telescope is then turned on and connected to The SkyX program on the computer which is how the slewing and pointing of the telescope are controlled. The telescope has a hand paddle, on which the time and date are entered, including whether 
or not you are in daylight savings. However, for some strange reason, to get the telescope to sync properly with the computer, the time entered on the telescope hand paddle must be the computer time (normal time) minus two hours. Finally, in CCDSoft, the "auto save" option is turned on so that all images taken during the night (except focusing images) are automatically numbered and saved with the specified prefix into the specified folder. Data collection can now begin.

Current issues with the telescope include dome tracking, telescope tracking, and setting the time on the telescope hand paddle. Because this telescope is relatively new, the dome software is still set to track the position of the old telescope. Due to differences in the mount and length of the new telescope, the dome will track the telescope, but it may not be perfect tracking. Depending on the part of the sky being viewed, the dome might not align perfectly with the new telescope, meaning the images will either be vignetted stars, or at worst, the inside of the dome. The second issue is telescope tracking. The telescope should be able to track the stars in sidereal stare mode, however, for many one minute exposures, the stars appeared more elliptical than circular. This seemed to happen more often when walking around inside or outside the dome while the image was being exposed, but also sometimes occurred randomly at other times. The other telescope tracking issue is that the telescope can only be slewed slowly across the sky from a bright star to another bright star, and must be re-centered along the way. This might not be a problem that can be permanently fixed, however, the distance that can be slewed before a star is not in the FOV at all could likely be increased if a little more time is spent aligning the telescope properly. This was not done as well as it should have been when the telescope was set up when it was installed. Finally, the time being entered into the key pad should not be two hours different than the actual time of day. No theories exist for this error because being only one hour off would suggest a daylight savings error, but even then, the keypad asks about daylight savings before asking for the time and date.

During data collection, rocket body targets were tracked with the SkyX software, however, there were issues with using this method for tracking. The cross-track error, which is the error in satellite position perpendicular to its trajectory, was usually minimal, or at least within the FOV for targets that were successfully observed. The along-track error is the satellite position error along its trajectory, and was off by anywhere from 45 seconds to 7 minutes for different targets that were observed. In each case, the satellite would pass through the FOV of the camera after (never before) the SkyX predicted it to. This was 
investigated, however, no solution was found. The computer clock matched the time being displayed in the SkyX program exactly, and both matched the time from a cell phone within seconds, therefore eliminating this as the possible source of error. The other theory tested was if the SkyX program rounded or truncated the epoch date found in the 3LE file. An error of 7 minutes could be caused by rounding or truncating the epoch date 0.005 days. Because all the targets appeared after the SkyX predicted them to pass by, the truncation theory was tested first and involved only keeping digits of the epoch date after the first two digits after the decimal, and comparing to the measured time behind. If a correlation existed then the truncation amount would exactly correspond to the amount of time the target was observed to be behind. Unfortunately a plot of the truncated decimal places vs. the measured along-track error was not a line, but a scatter plot, showing this was not the source of the error. The next approach tried was the rounding approach. The same truncated decimal places were taken, but were subtracted from 0.01 if it was larger than 0.005 days. This showed more of a correlation than the truncation theory, however, the differences between rounded dates and measured along-track error times were still over a minute for most and close to three and four minutes for two in particular. Measurement of the observed along-track error was not precise to the second, but it was certainly on the order of at most several seconds, which is still smaller than several minutes, therefore also disproving this theory.

Once all data has been collected for the night the telescope is parked in its home position, then is disconnected from The SkyX and switched off. Once covers for the main lens and for the finder scope are replaced on the telescope, the tarp is put over the top of the telescope to protect it from dust. The camera (and focuser) are disconnected from the camera software, and then each are powered off. The dome must be slewed to its home position using the manual controls on the wall because the DDW software has problems getting to the home position. Only once in the home position can the dome be closed! After making sure all data and images for the night have been saved, a file transfer window is opened and image data is transferred to a computer named "planet" which is the computer that can run IRAF software for data reduction. Finally, the observatory computer is shut down and the main power switch on the wall for dome power is turned off. Once the observatory is locked up it is time to go home and sleep! 


\section{APPENDIX D: Epar Settings Used in IRAF}

Table 7. Epar rfits Settings

\begin{tabular}{|c|c|}
\hline fits_fil & $*$.FIT \\
\hline file_lis & \\
\hline iraf_fil & <image prefix> \\
\hline make_im & yes \\
\hline long-he & no \\
\hline short_h & yes \\
\hline datatyp & \\
\hline blank & 0 \\
\hline scale & yes \\
\hline oldiraf & no \\
\hline offset & 0 \\
\hline
\end{tabular}

A description of the rfits ${ }^{[9]}$ command and its settings can be found on the Space Telescope Science Data Analysis System help page.

Table 8. Epar Zero, Epar Dark, and Epar Flat Settings

\begin{tabular}{|l|l|l|}
\hline epar & zerocombine & flatcombine \\
\hline Input & $*$.fits & *.fits \\
\hline Output & masterbias & masterflat \\
\hline Combine & average & median \\
\hline Reject & minmax & avsigclip \\
\hline Ccdtype & zero & flat \\
\hline Process & no & yes \\
\hline Subsets & n/a & yes \\
\hline Delete & no & no \\
\hline Clobber & no & no \\
\hline Scale & none & mode or median \\
\hline Statsec & & {$[700: 1400,400: 1100]$} \\
\hline Nlow & 0 & 1 \\
\hline Nhigh & 1 & 1 \\
\hline Nkeep & 1 & 1 \\
\hline Mclip & yes & yes \\
\hline Lsigma & 3 & 3 \\
\hline Hsigma & 3 & 3 \\
\hline Rdnoise & 8.8 & 8.8 \\
\hline Gain & 1.3 & n/a \\
\hline
\end{tabular}

A description of the zerocombine ${ }^{[15]}$ and flatcombin ${ }^{[7]}$ commands and their respective settings can be found on the Space Telescope Science Data Analysis System help page. 
Table 9. Epar Ccdproc Settings

\begin{tabular}{|l|l|l|}
\hline ccdproc & zero & flat \\
\hline Images & $*$.fits & $*$.fits \\
\hline Max_cac & 0 & 0 \\
\hline Noproc & no & no \\
\hline Fixpix & no & no \\
\hline Oversca & no & no \\
\hline Trim & no & no \\
\hline Zerocor & yes & no \\
\hline Darkcor & no & no \\
\hline Flatcar & no & yes \\
\hline Illumco & no & no \\
\hline Fringec & no & no \\
\hline Readcor & no & no \\
\hline Scancor & no & No \\
\hline ccdproc (cont.) & zero & flat \\
\hline Readaxi & line & line \\
\hline Zero & masterbias & \\
\hline Dark & & \\
\hline Flat & & masterflatClear \\
\hline Minrepl & 1 & 1 \\
\hline Scantyp & shortscan & shortscan \\
\hline Nscan & 1 & 1 \\
\hline Interac & no & no \\
\hline Functio & 1 & legendre \\
\hline Order & 1 & 1 \\
\hline Sample & & $*$ \\
\hline Naverag & 1 & 1 \\
\hline Niterat & \\
\hline & 1 & \\
\hline
\end{tabular}

A description of the $\operatorname{ccdproc}^{[3]}$ command and its settings can be found on the Space Telescope Science Data Analysis System help page. 


\section{APPENDIX E: Selected Light Curves for Analyzed Data}

Presented here are a sample of the light curves that were generated from image analysis. The plots are shown with brightness increasing upward along the y-axis, therefore magnitude values increase downward along the y-axis. The plots are ordered first by object SSN number, then by date if multiple observations were taken. First a plot of the light curve is shown for an entire group of images, followed by a zoomed in plot of just a single light curve for one individual image. Else it is impossible to see the periodic nature of the tumbling rocket bodies. For the graph of the light curve for an entire set of images, just dots are shown where magnitude values are located, however, for the zoomed in images, the dots are connected with lines. This is because it is impossible to see the periodic motion in the graph without lines connecting the data points; the graph simply looks like a scatter plot without the lines.

Once again, the calibration from pixel brightness to instrumental magnitude is NOT correct, as there is no way to properly calibrate the C value of Eqn. (1) without a filter. A "C" value of 24.9921 was used. Because the $\mathrm{C}$ value serves only to shift the light curve up or down, the differential magnitude between bright and dim peaks of the light curves can be taken as the actual change in brightness over time.

\section{SSN 36034, INTLDES 2009-058C}

Target 36034 was captured in 20 images on the first night, with about an hour in between each set of ten images, and all twenty were kept. On the second night, the same twenty pictures were taken, but with two hours between each set of ten images, however, only five images of the last set of ten were kept. 
Light Curve
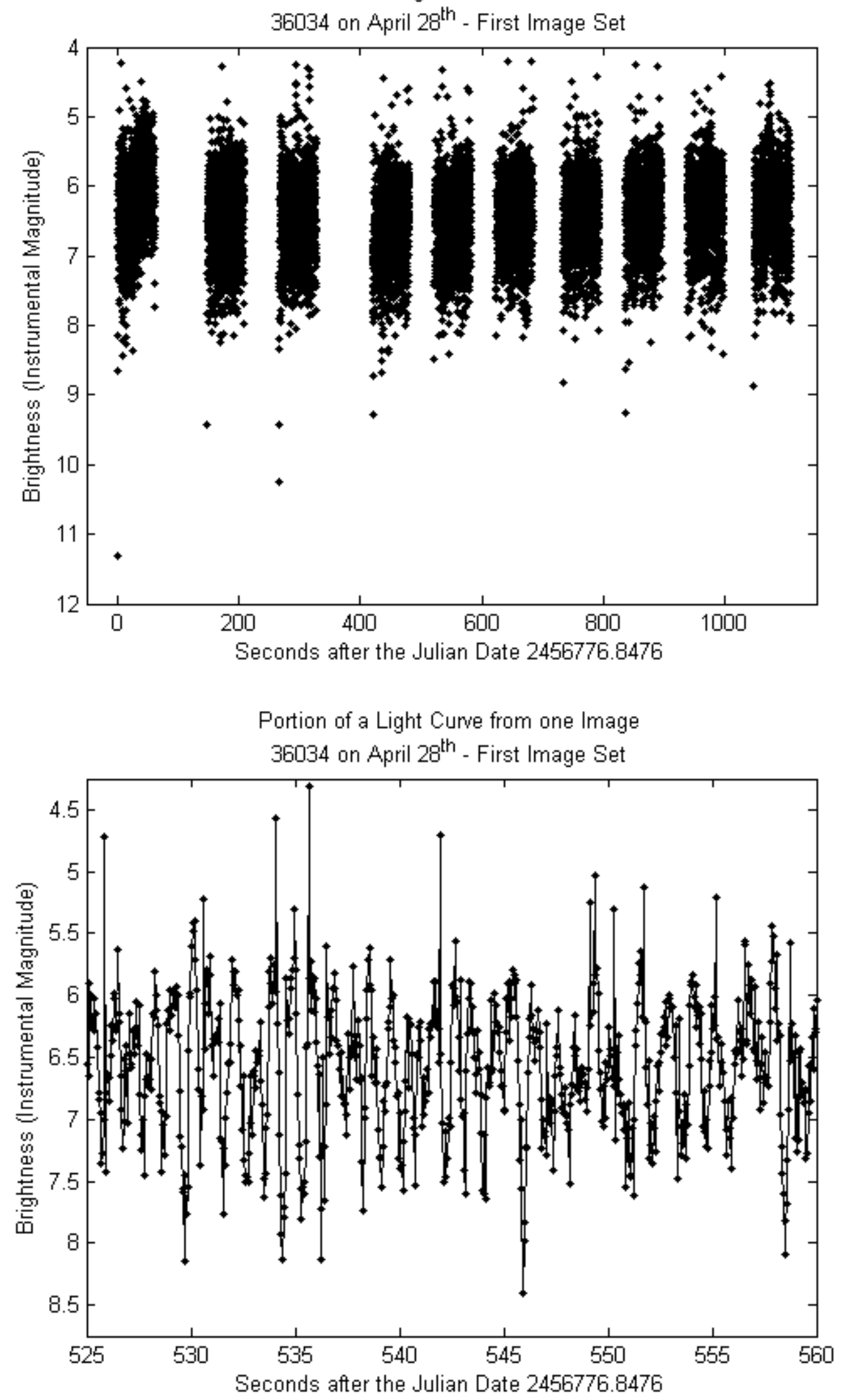
Light Curve

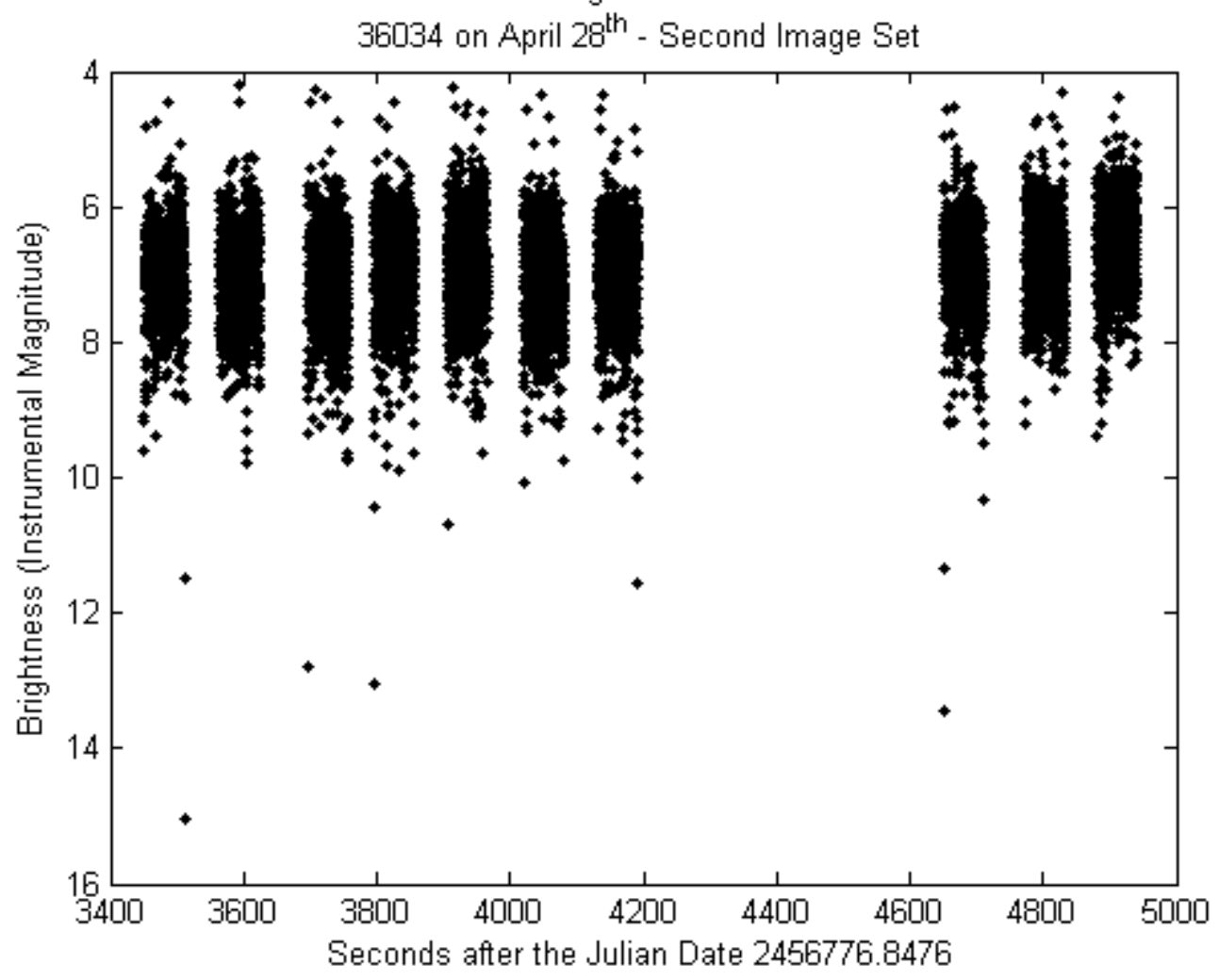

Portion of a Light Curve from one Image

36034 on April $28^{\text {th }}$ - Second Image Set

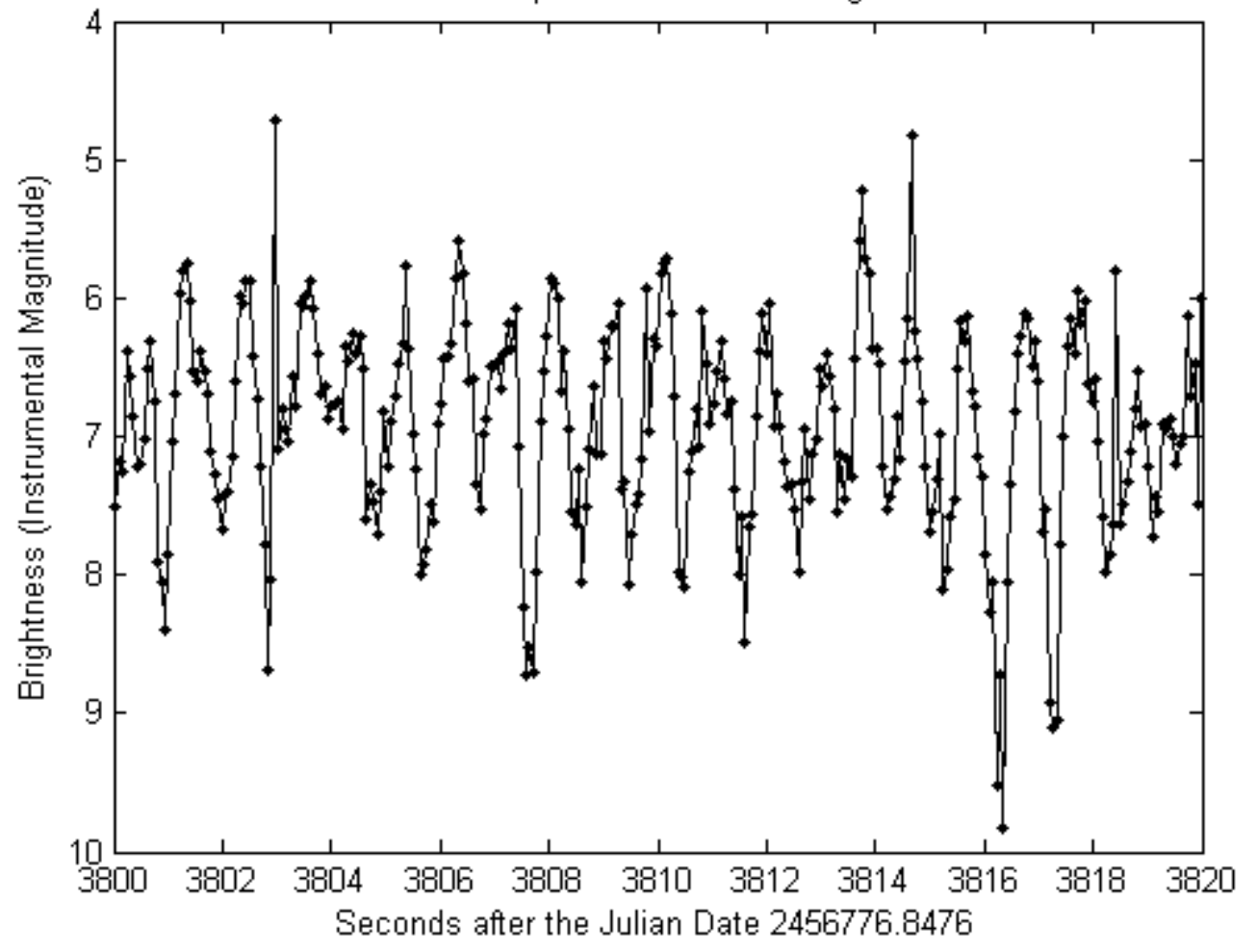


Light Curve
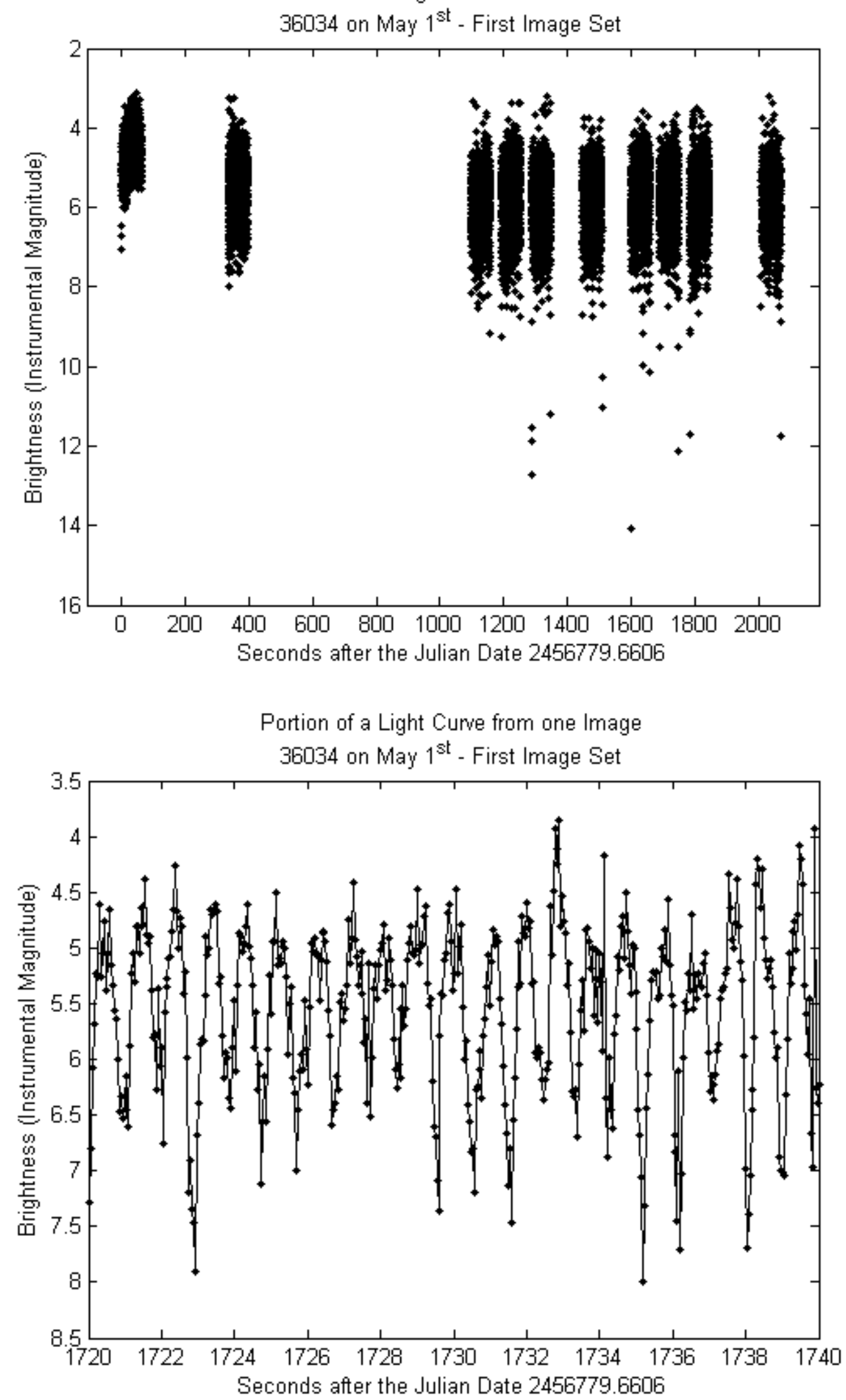
Light Curve

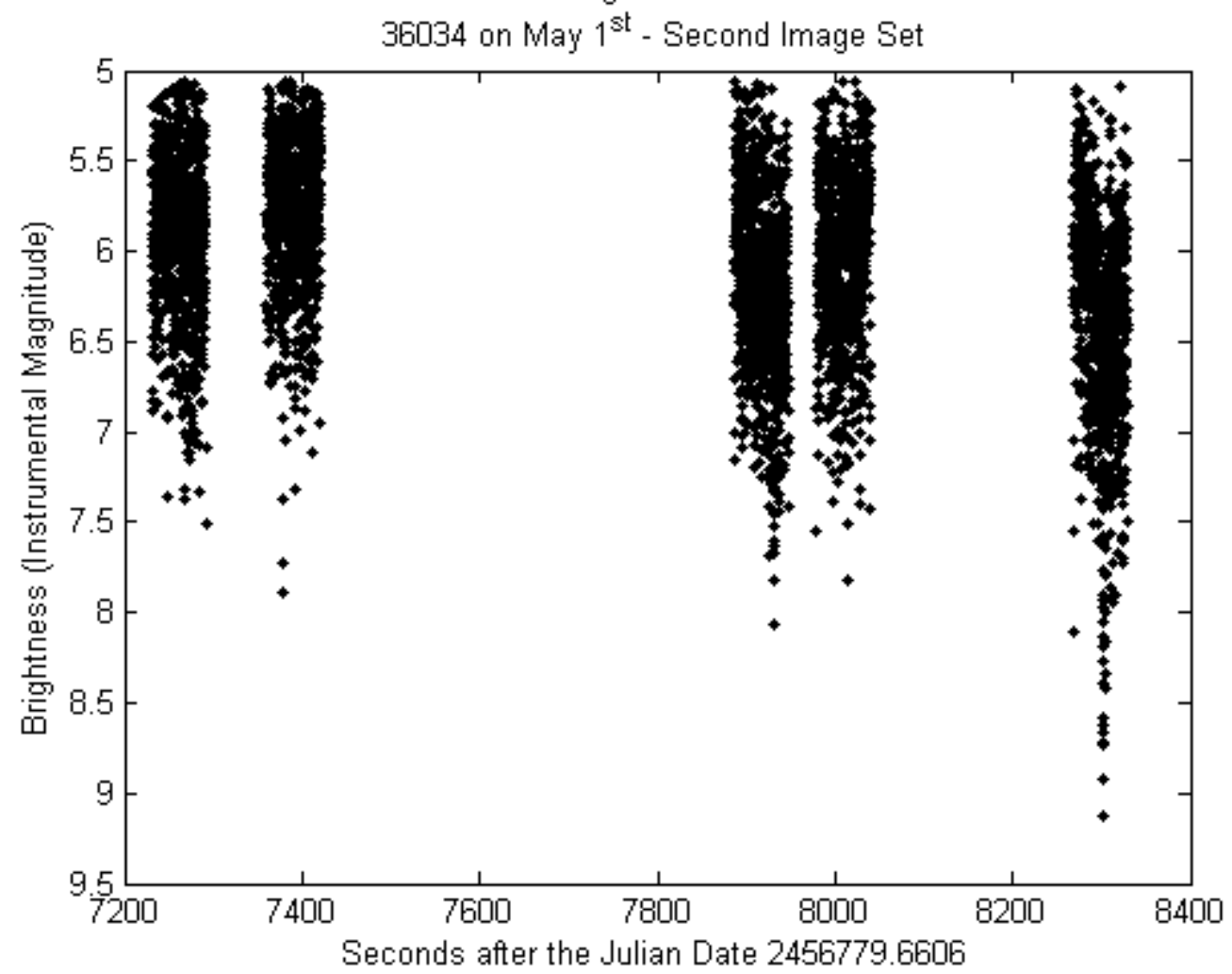

Portion of a Light Curve from one Image 36034 on May $1^{\text {st }}$ - Second Image Set

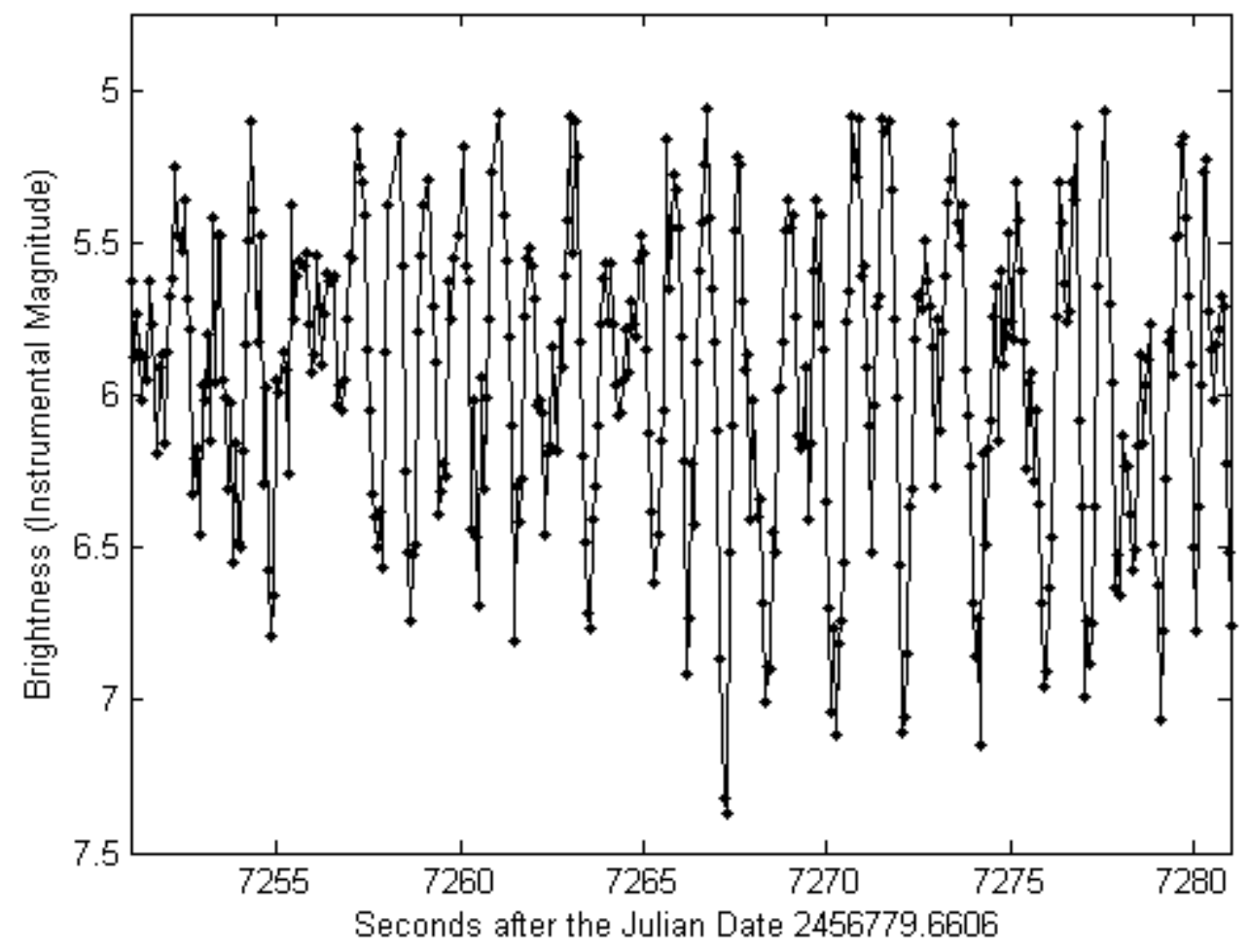




\section{2. $\operatorname{SSN} 37777$, INTLDES 2011-041C}

This target was captured on two separate nights. The first night ten images were obtained, but only five of those were kept for data reduction and analysis. The second night, another single set of ten images were taken, but only nine were kept.

Light Curve

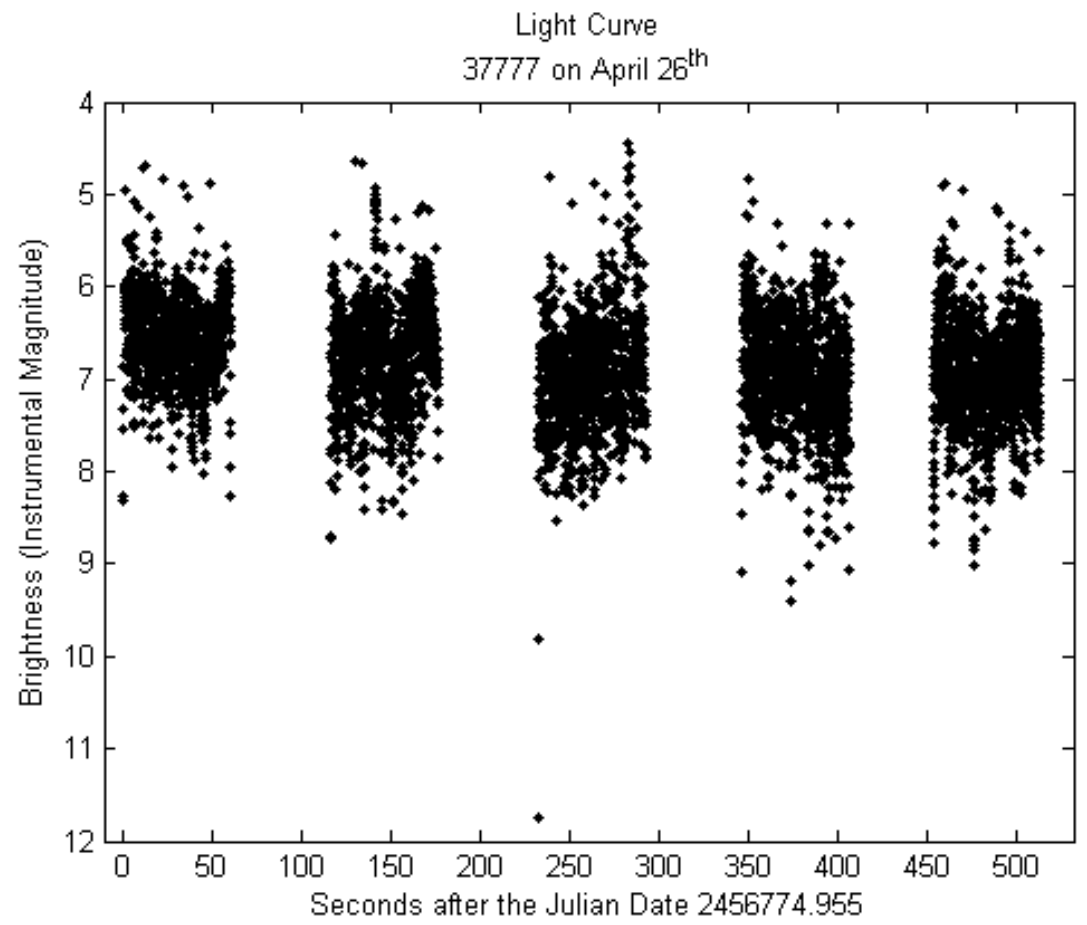

Portion of a Light Curve from one Image 37777 on April $26^{\text {th }}$

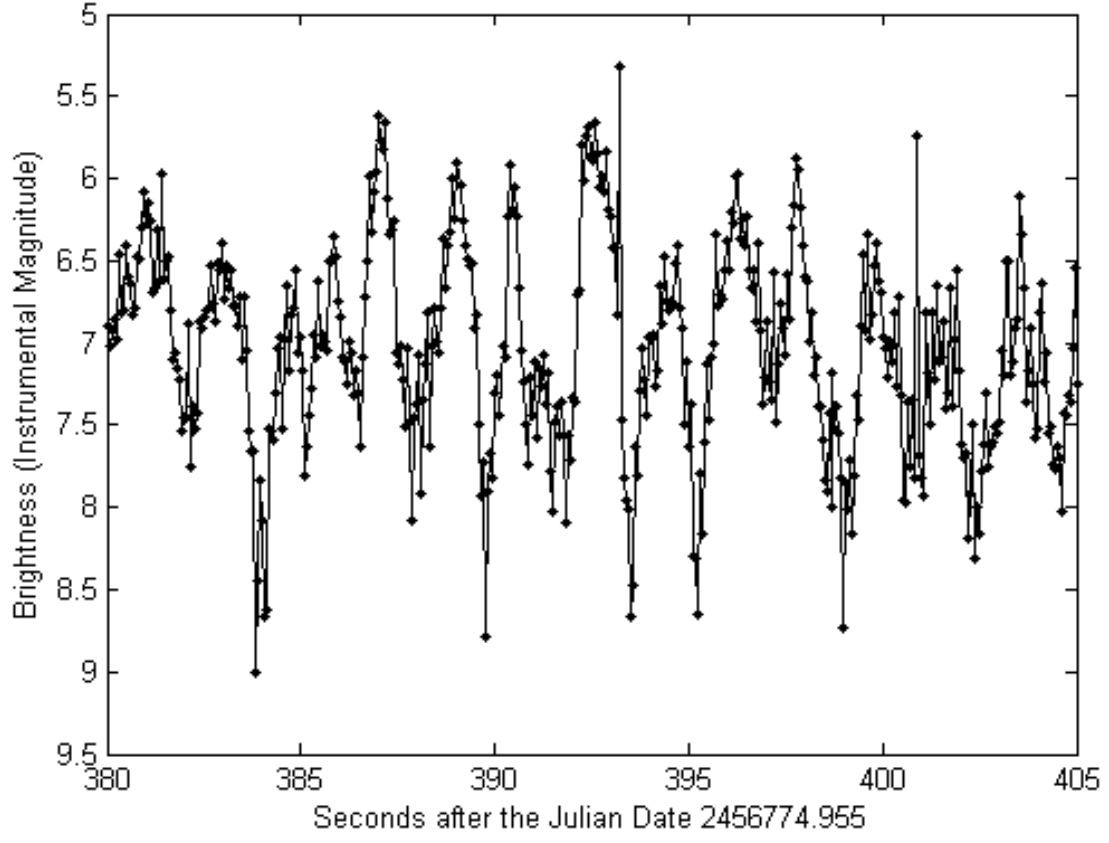


Light Curve

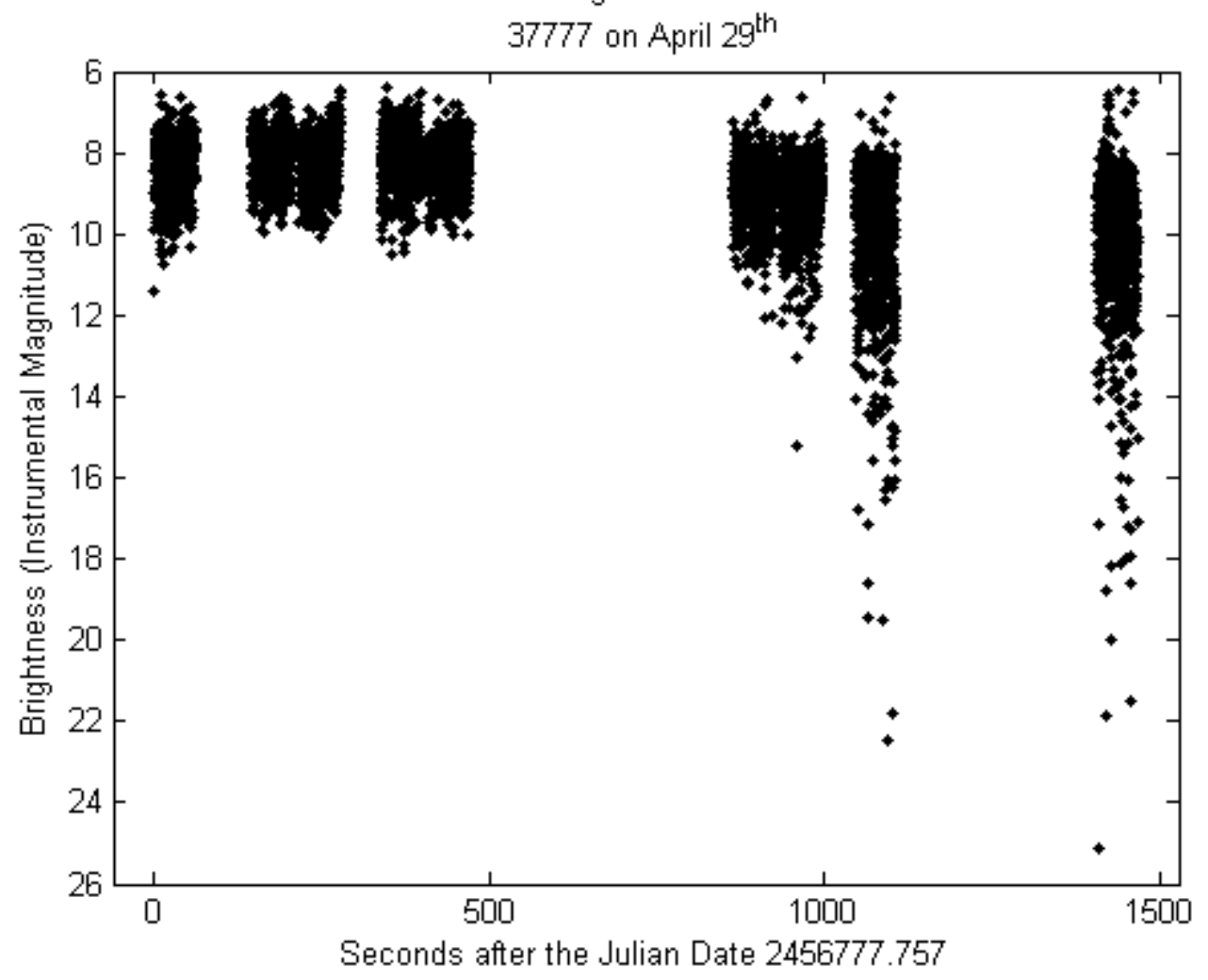

Portion of a Light Curve from one Image

37777 on April $29^{\text {th }}$

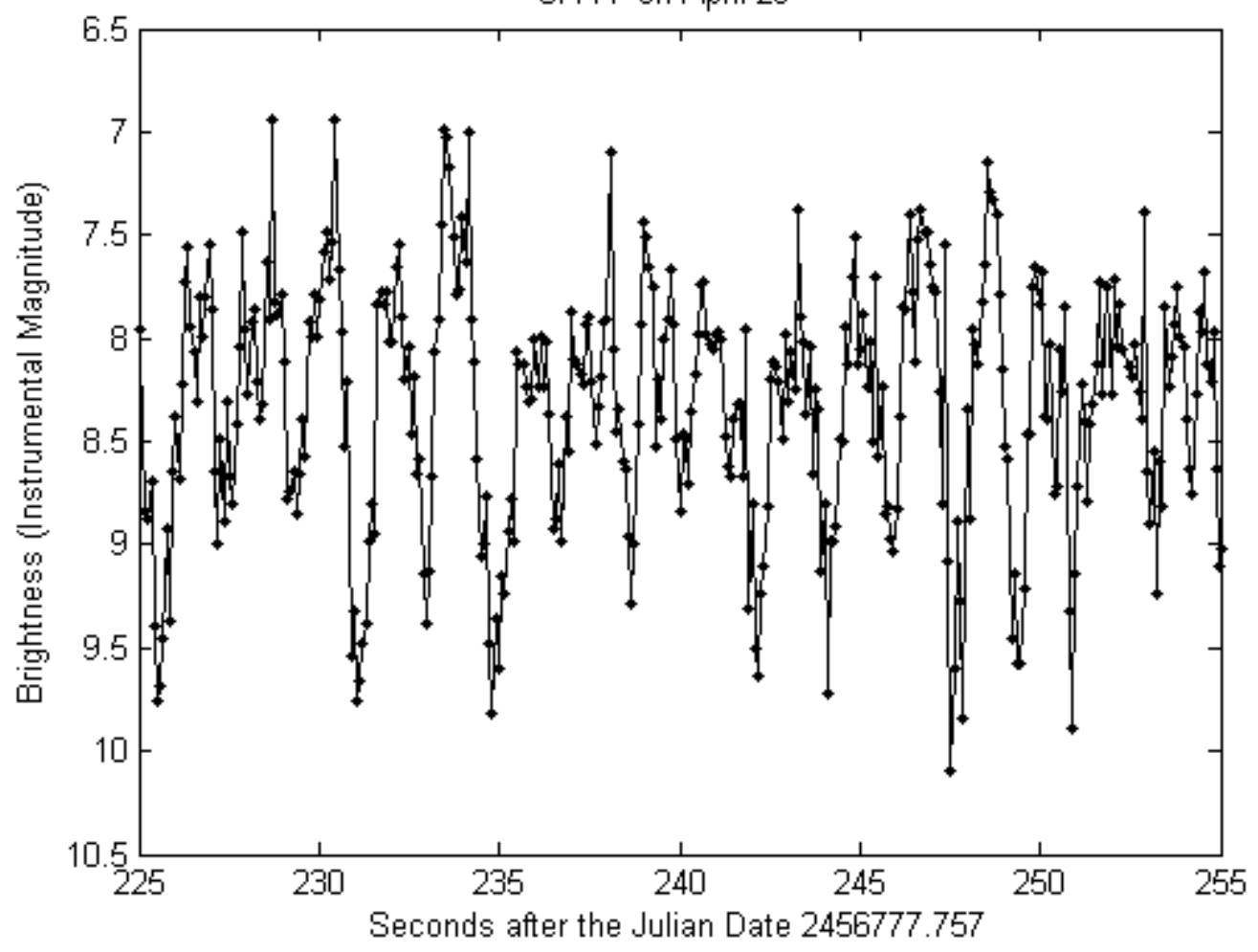




\section{SSN 37811, INTLDES 2011-049C}

Target 37811 was captured on three different nights. The first night was the very first night of data and thirteen images were taken. The second night, two sets of ten images were taken with over two hours in between. A third attempt was made but its altitude was not high enough, and it therefore streaked through the FOV in less than a minute and was too fast to capture. A successful third attempt was made on the final night of observation, again with two sets of ten images separated by over two hours.
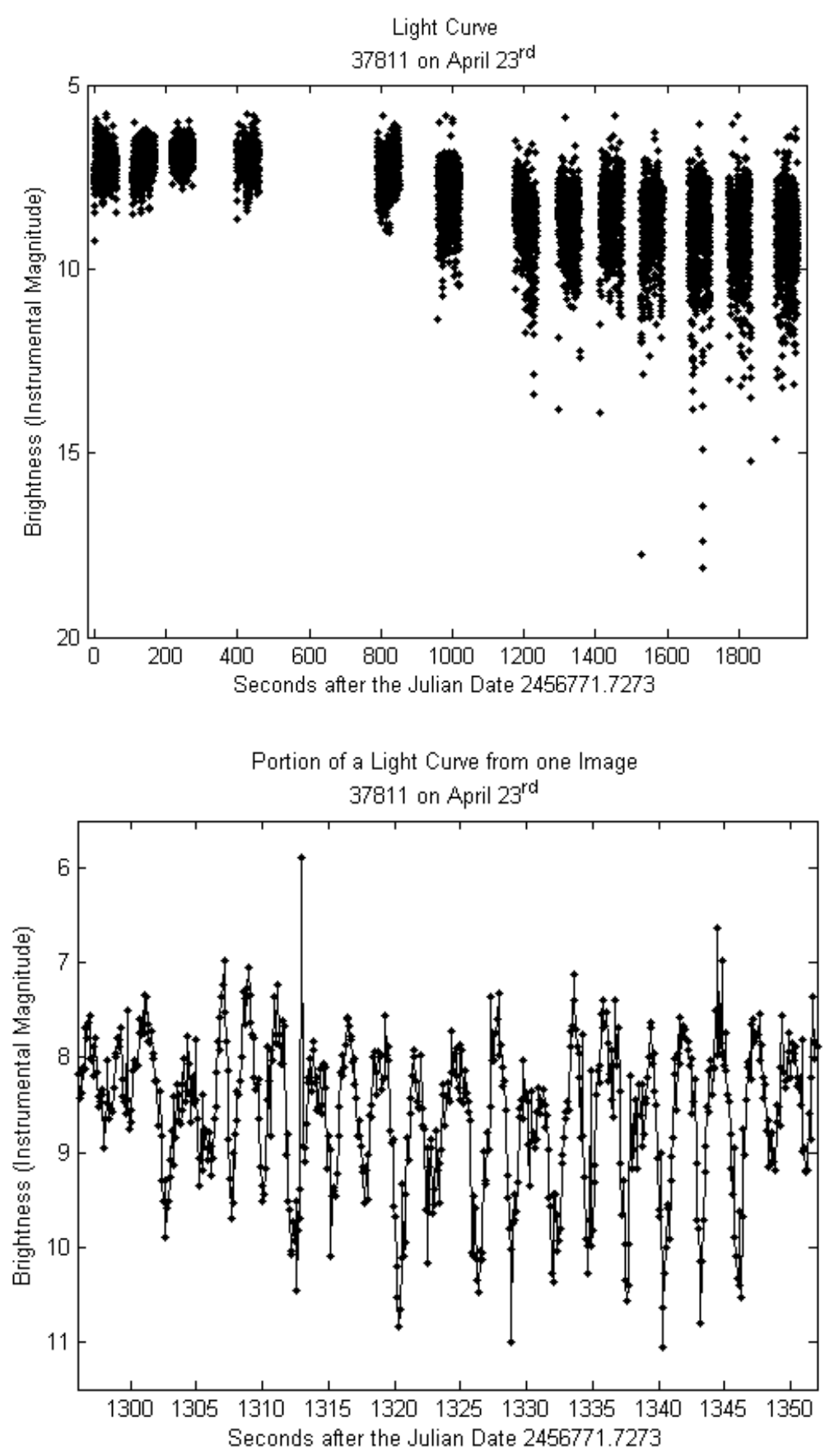
Light Curve

37811 on April $28^{\text {th }}$ - First Image Set

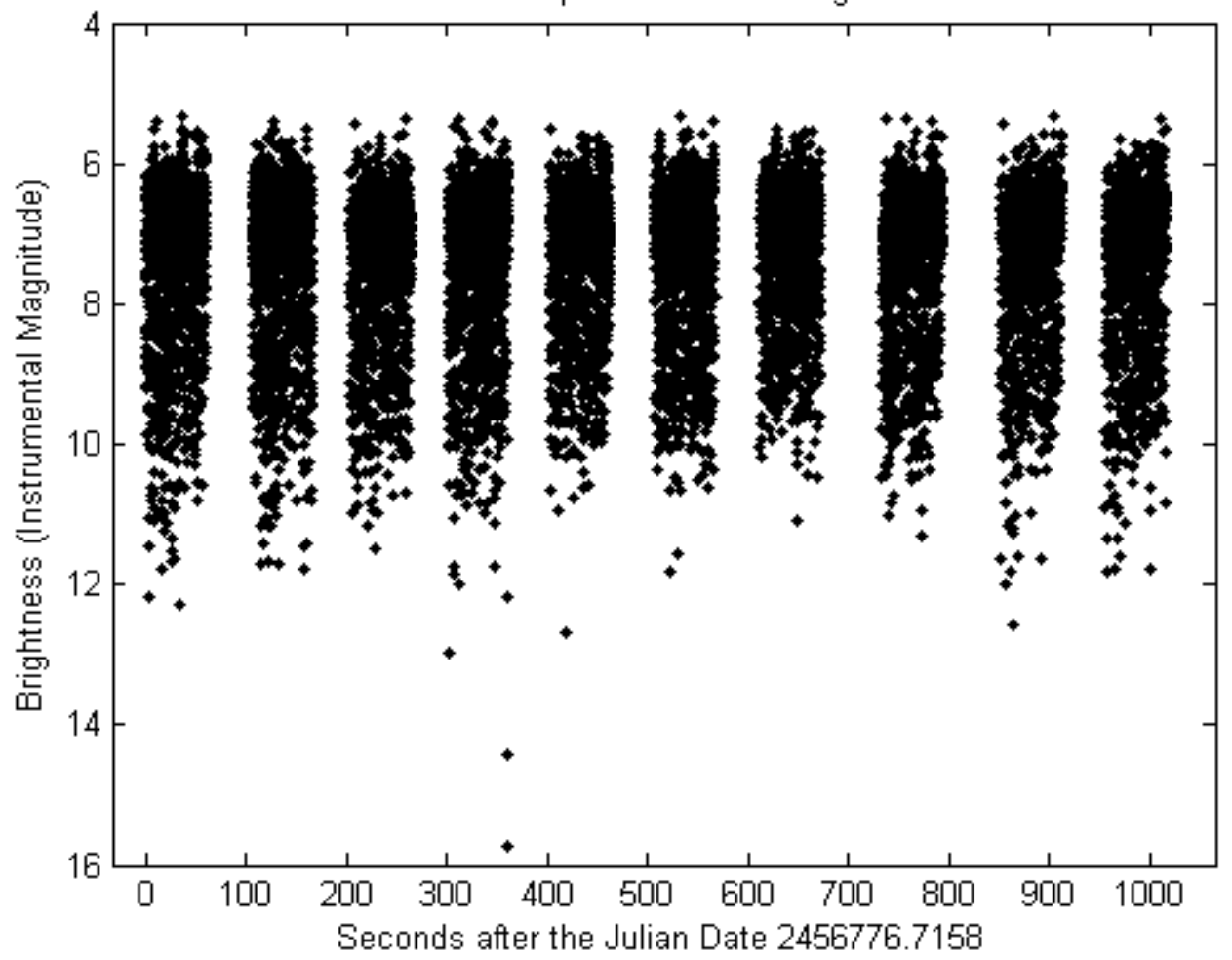

Portion of a Light Curve from one Image 37811 on April $28^{\text {th }}$ - First Image Set

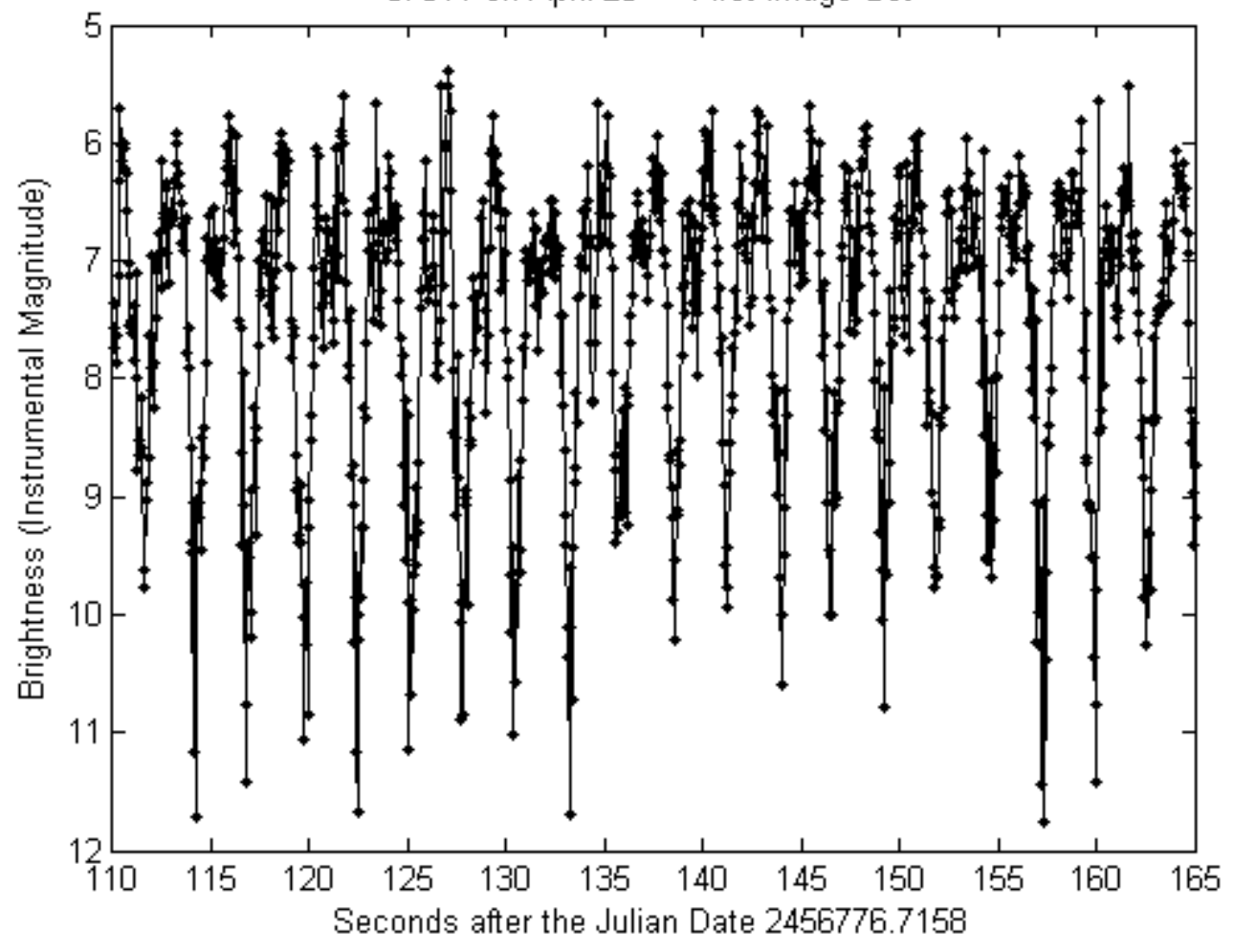


Light Curve

37811 on April $28^{\text {th }}$ - Second Image Set

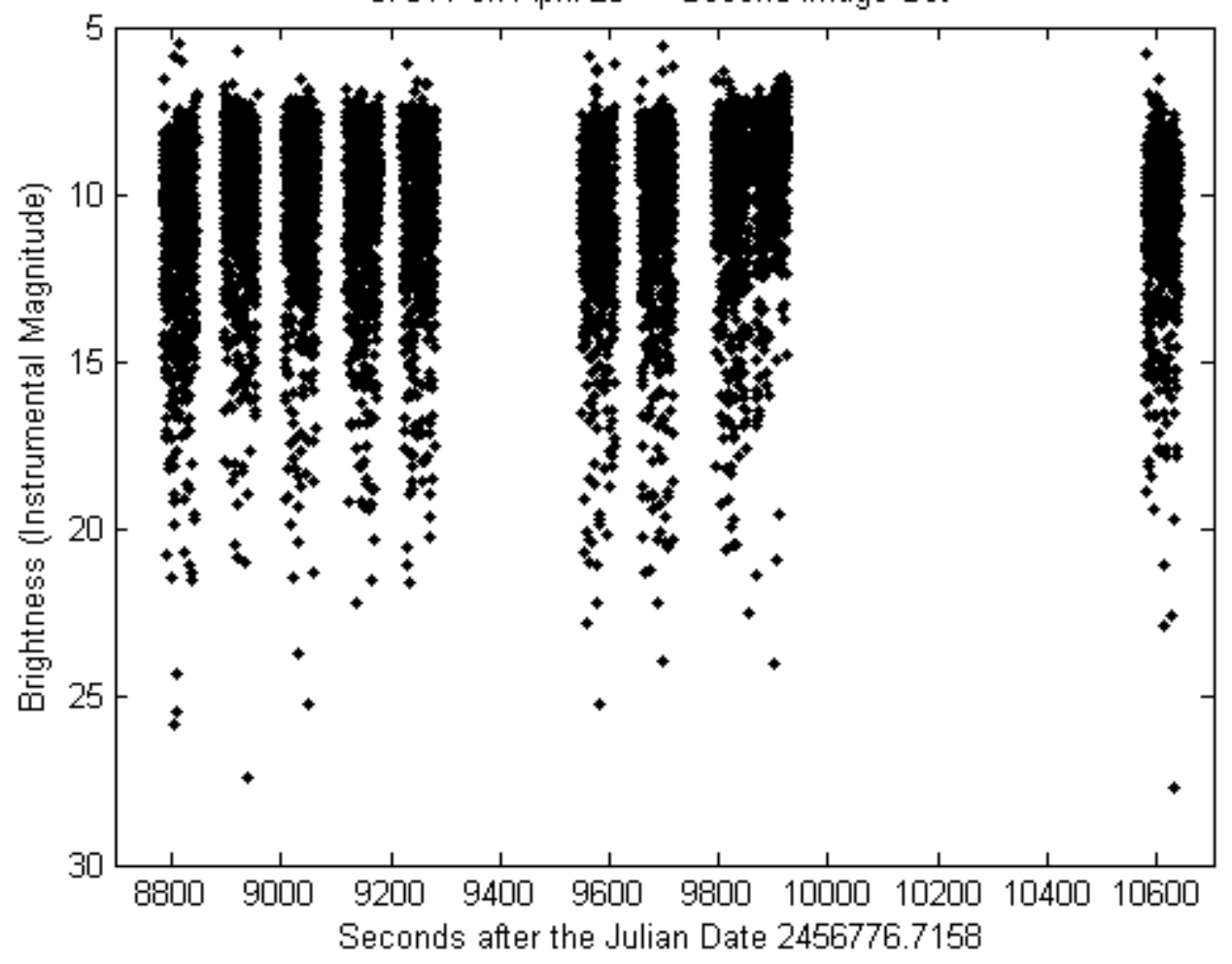

Portion of a Light Curve from one Image

37811 on April $28^{\text {th }}$ - Second Image Set

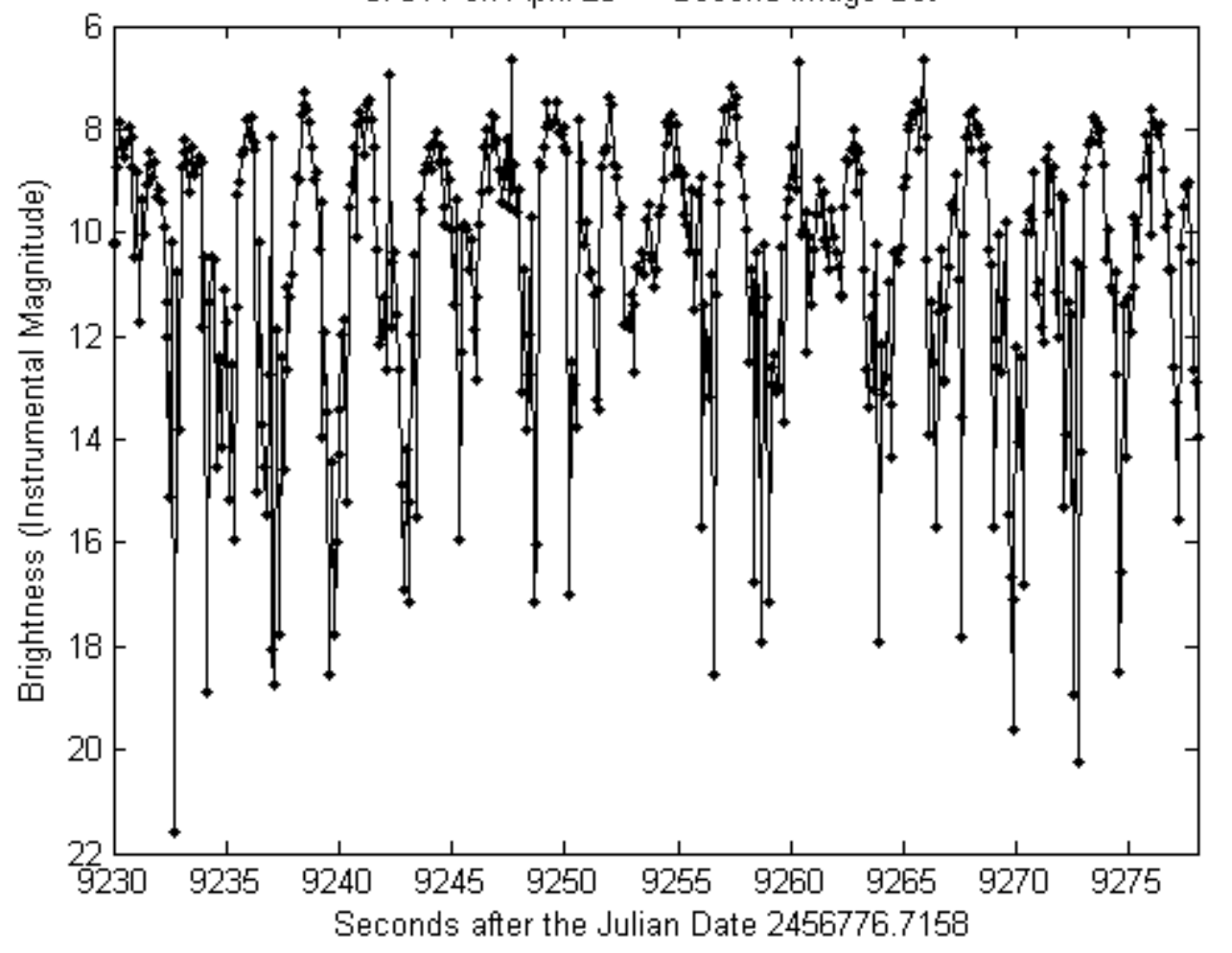


Light Curve

37811 on May $1^{\text {st }}$ - First Image Set

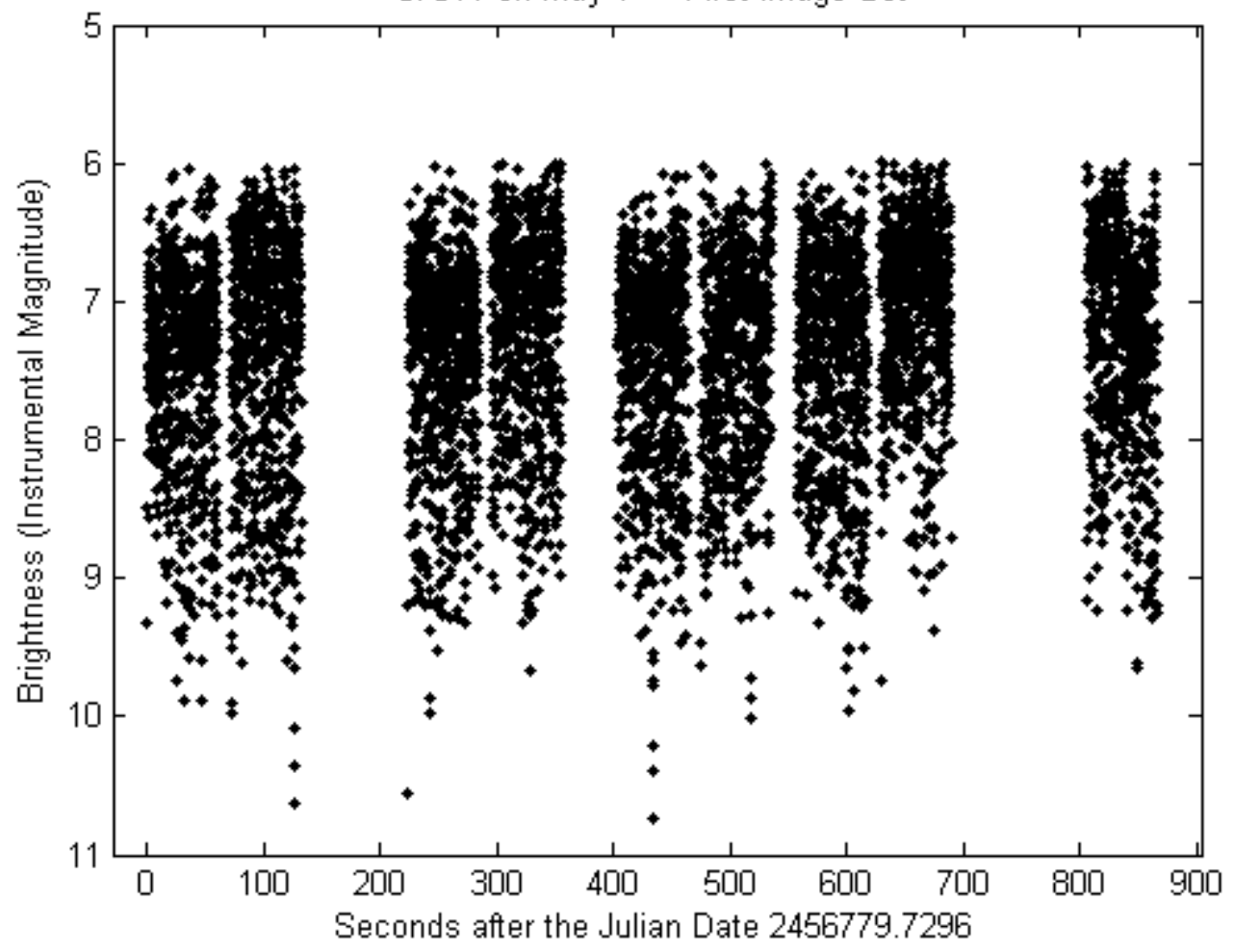

Portion of a Light Curve from one Image

37811 on May $1^{\text {st }}$ - First Image Set

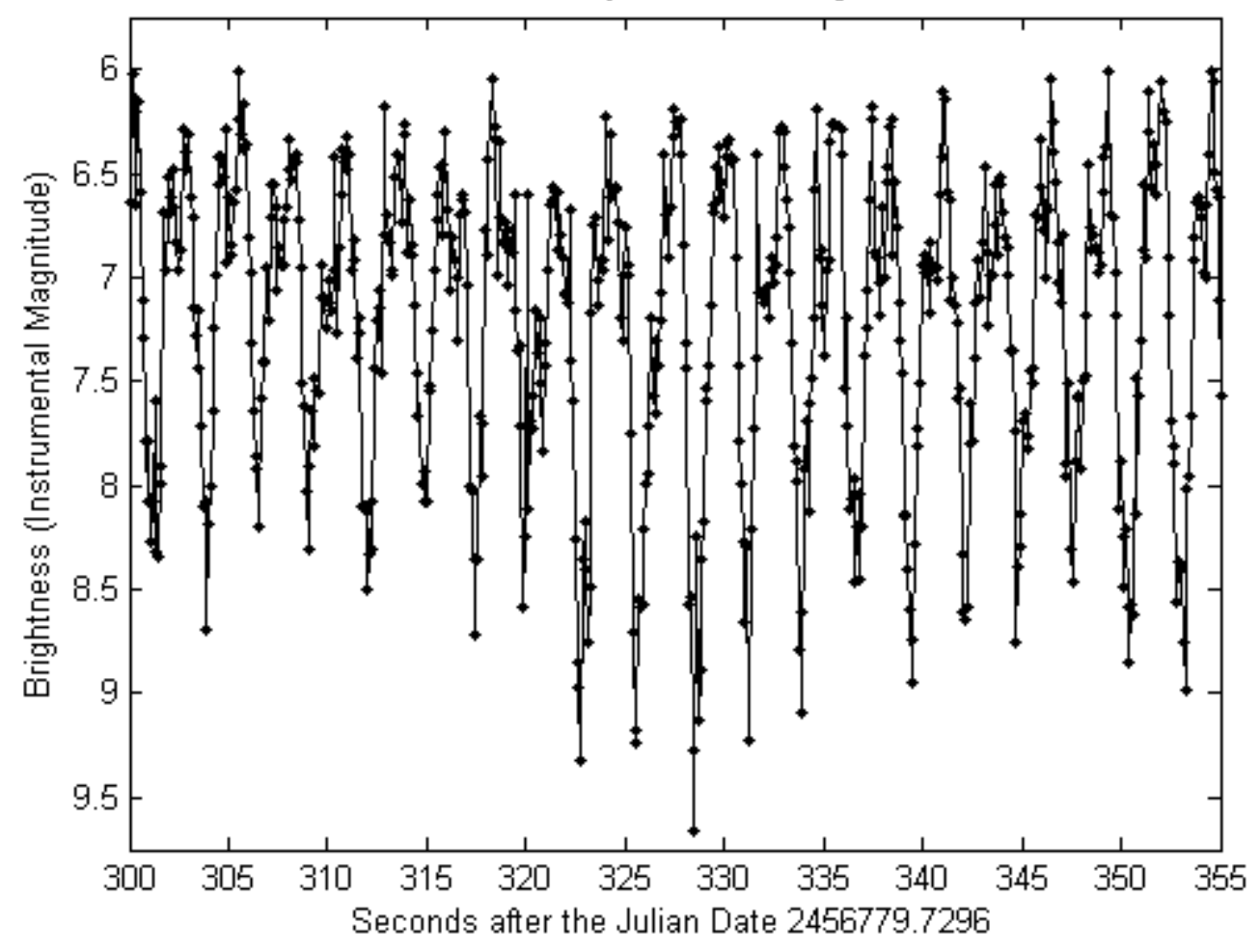


Light Curve

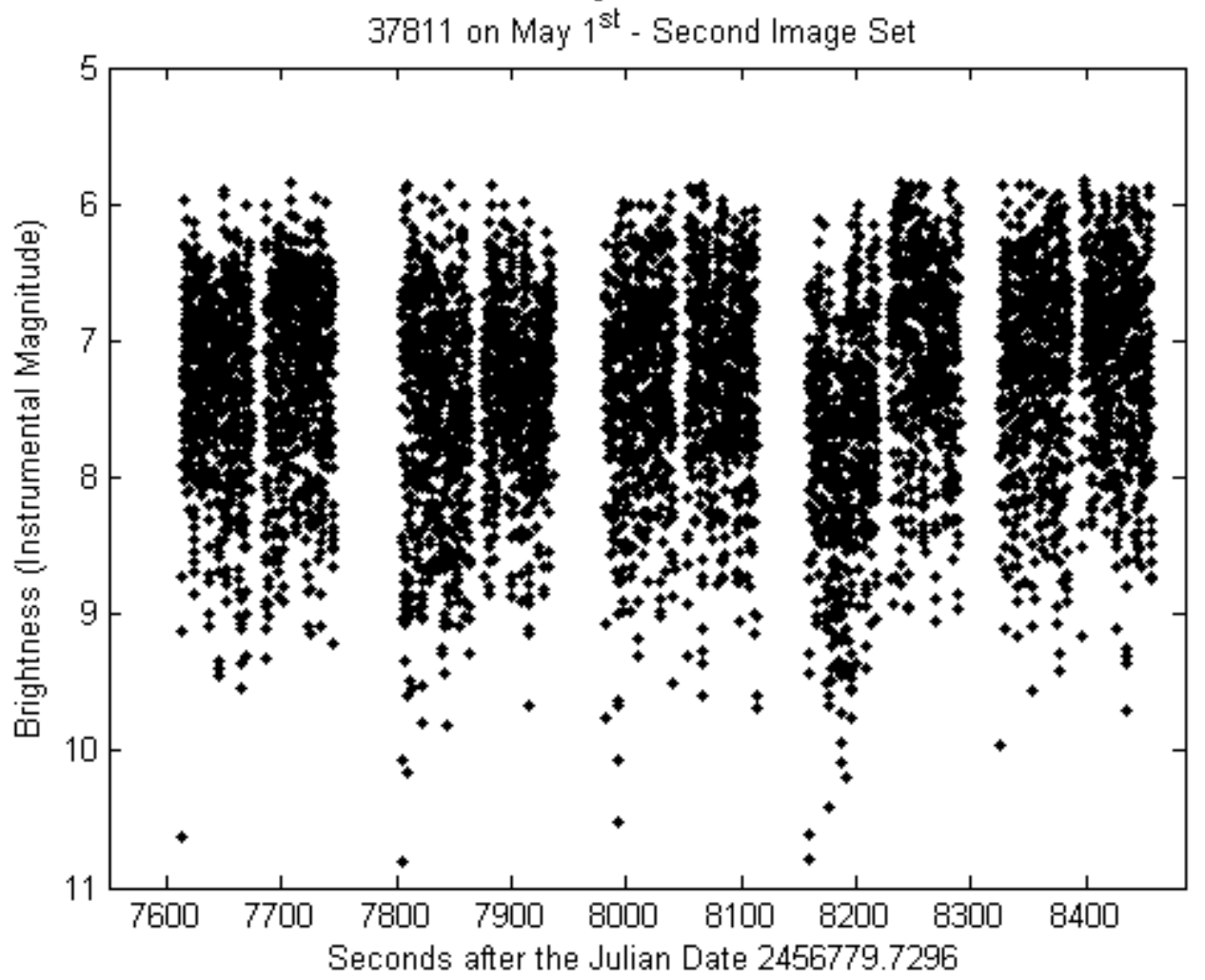

Portion of a Light Curve from one Image

37811 on May $1^{\text {st }}$ - Second Image Set

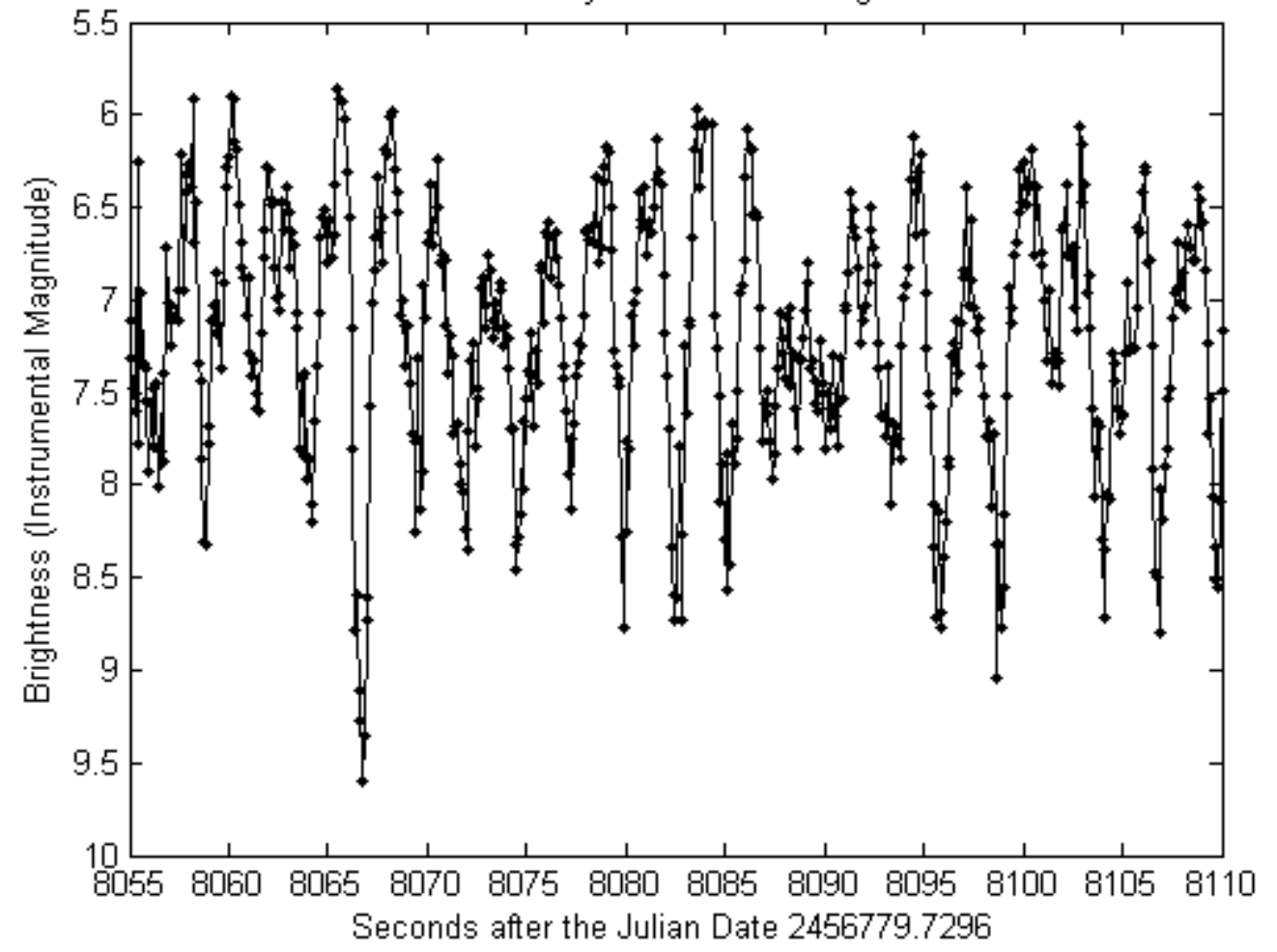


Ten images were obtained and kept from the first night of data collection. The next observation of this target was three nights later in which another ten images were obtained and kept. The final observation of this object was made another three nights later, but only five of the eight images were kept.

Light Curve

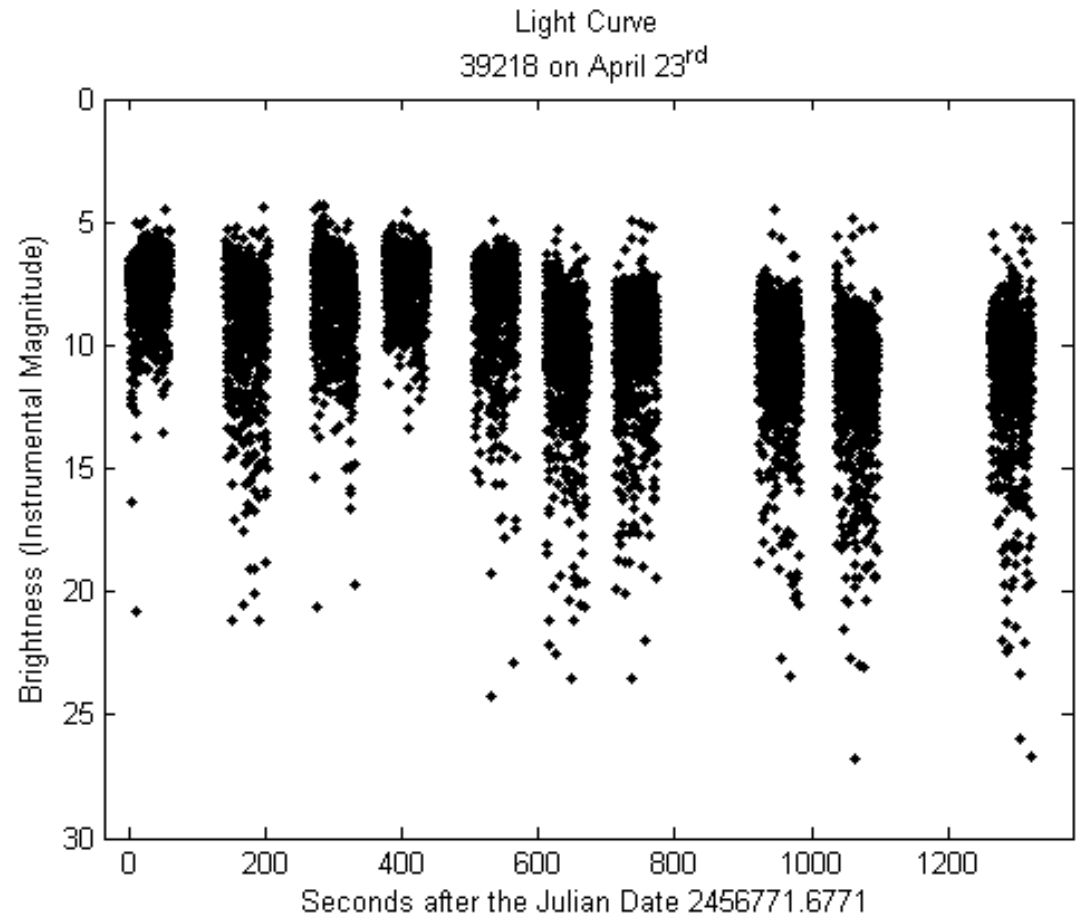

Portion of a Light Curve from one Image 39218 on April $23^{\text {rd }}$

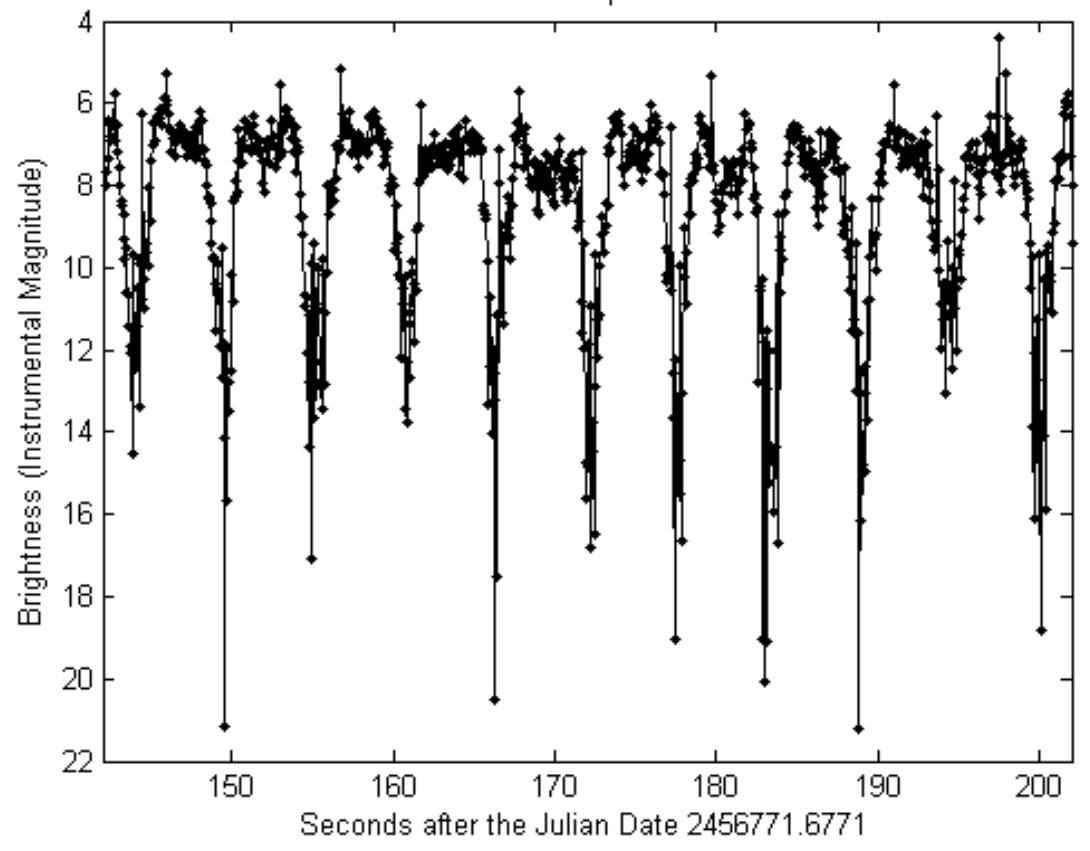


Light Curve

39218 on April 26 ${ }^{\text {th }}$

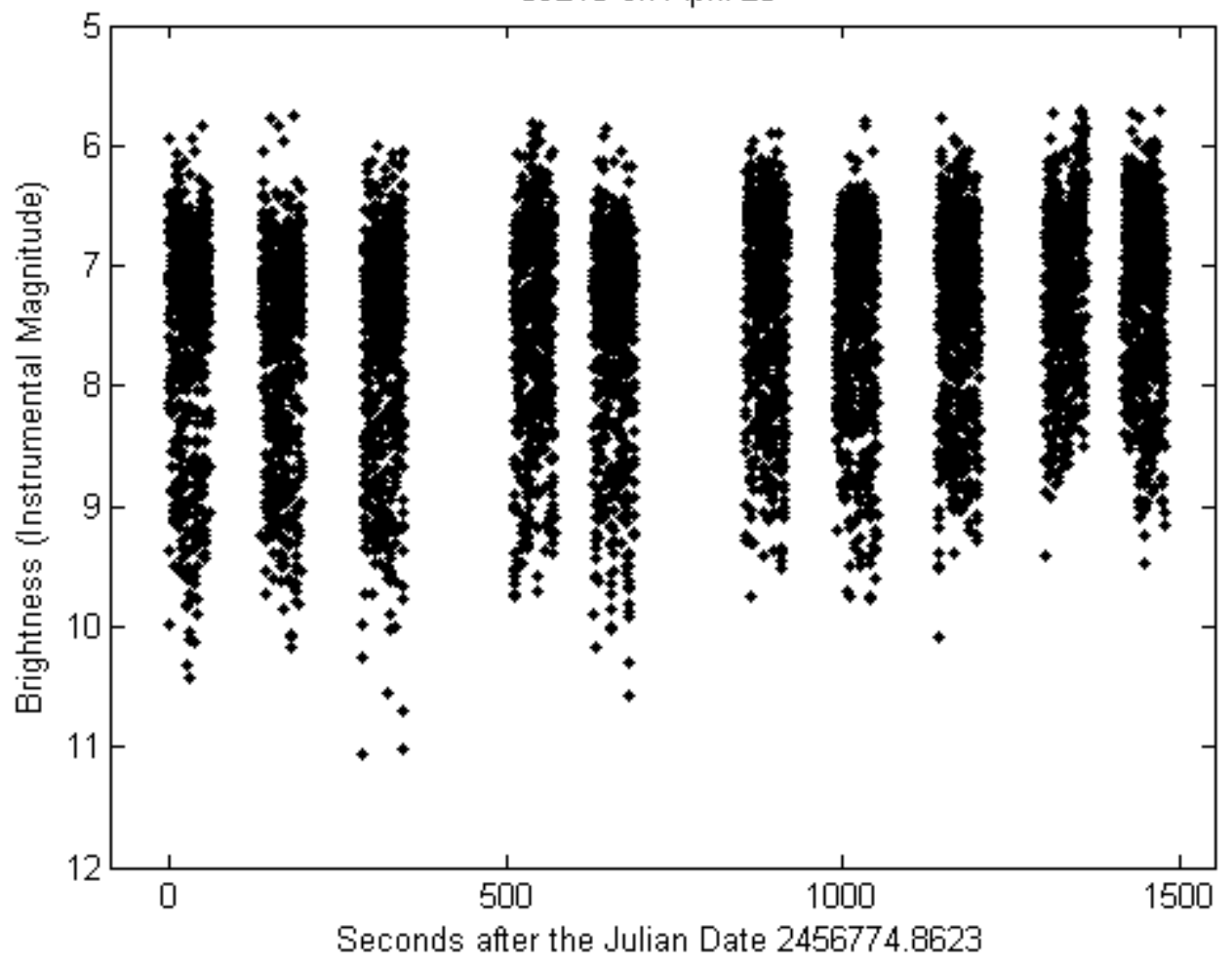

Portion of a Light Curve from one Image 39218 on April $26^{\text {th }}$

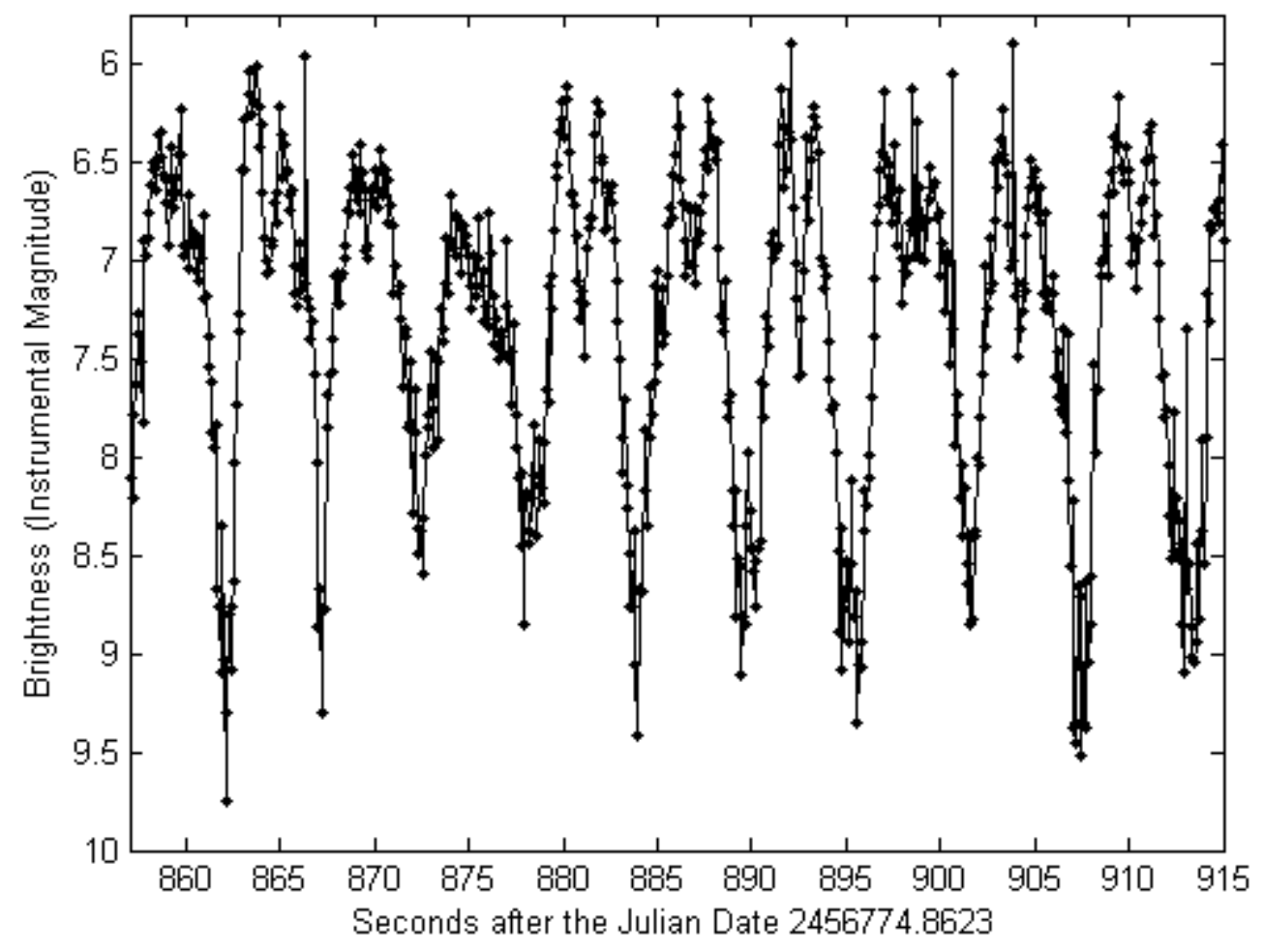


Light Curve
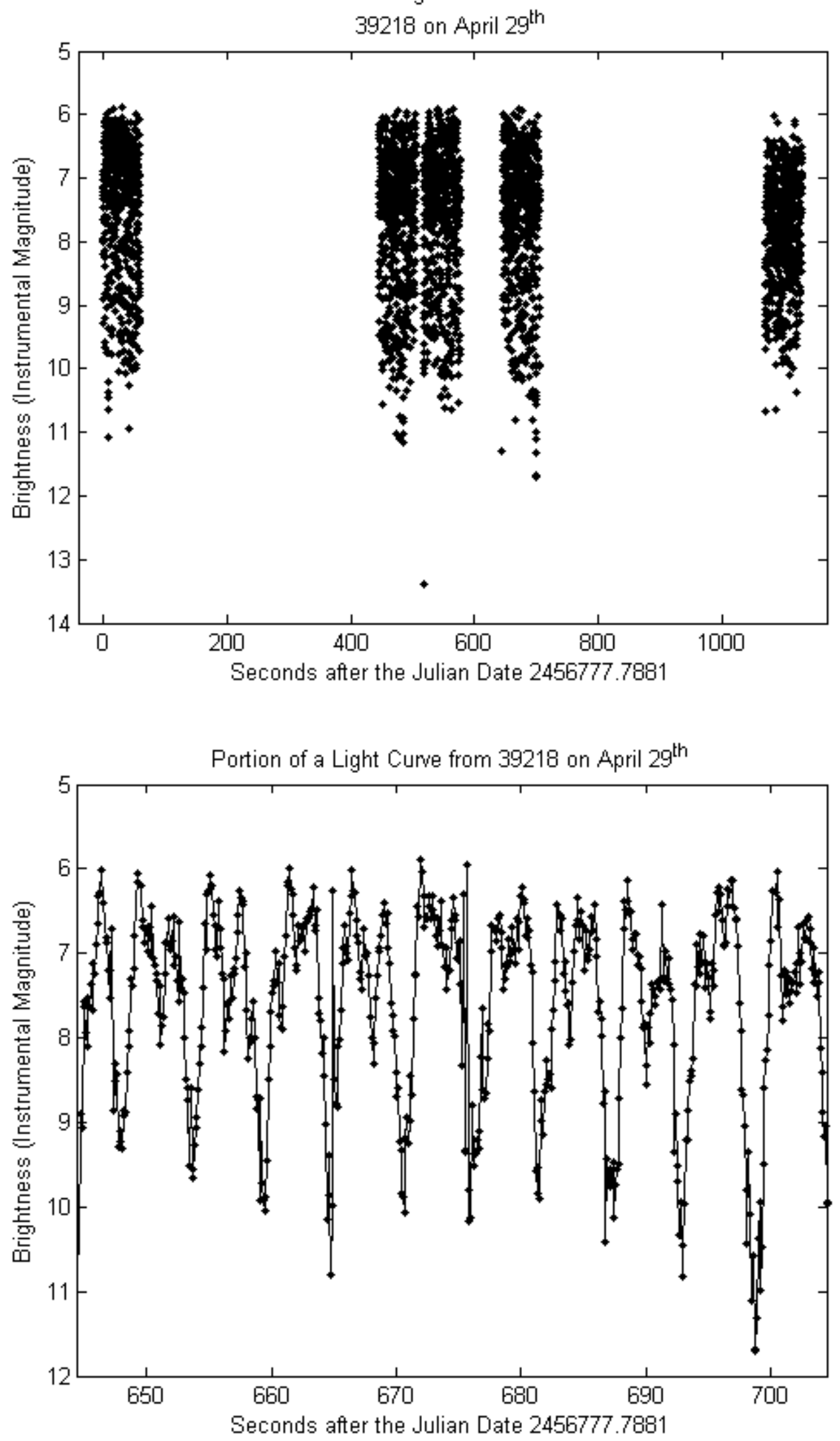


\section{SSN 39235, INTLDES 2013-044C}

This object was only successfully observed on one night but ten good images were taken that were all kept, and two hours later these were followed by ten more images, nine of which were kept. An attempt was made to capture this target the following night however it was unsuccessful.

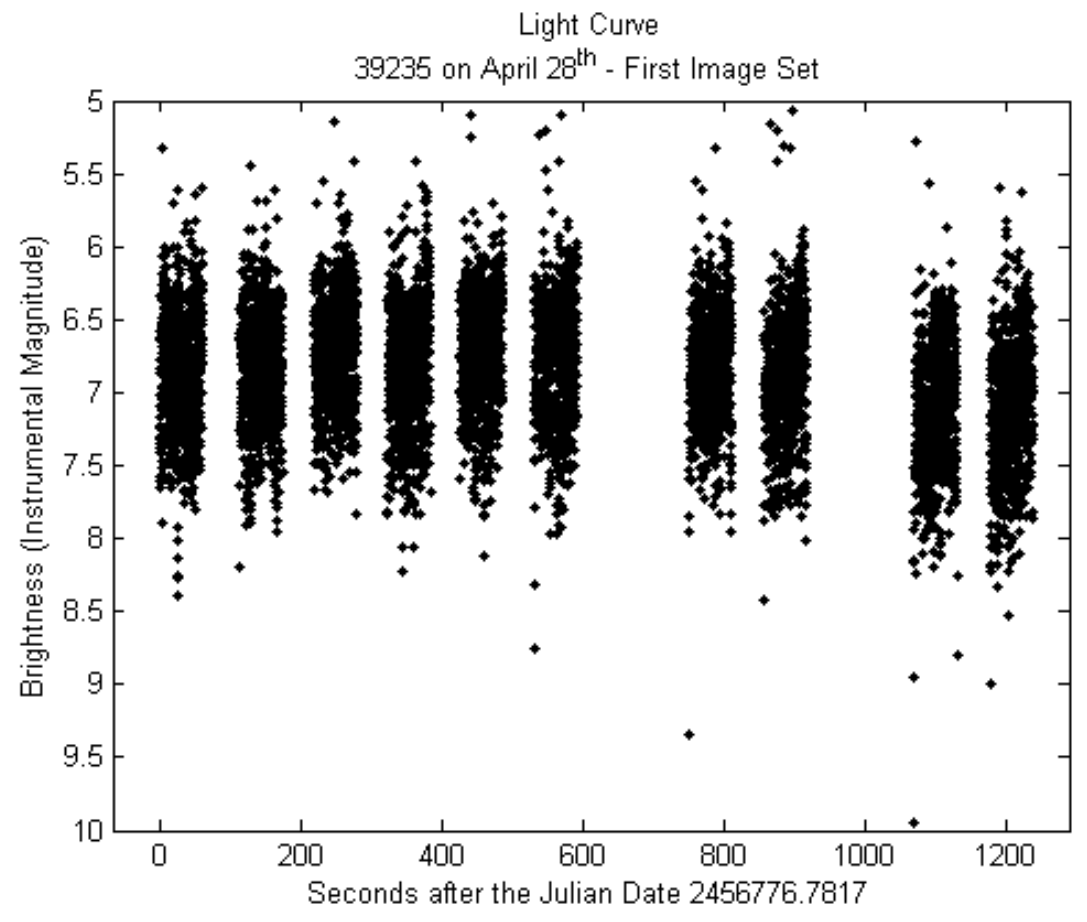

Portion of a Light Curve from one Image

39235 on April $28^{\text {th }}$ - First Image Set

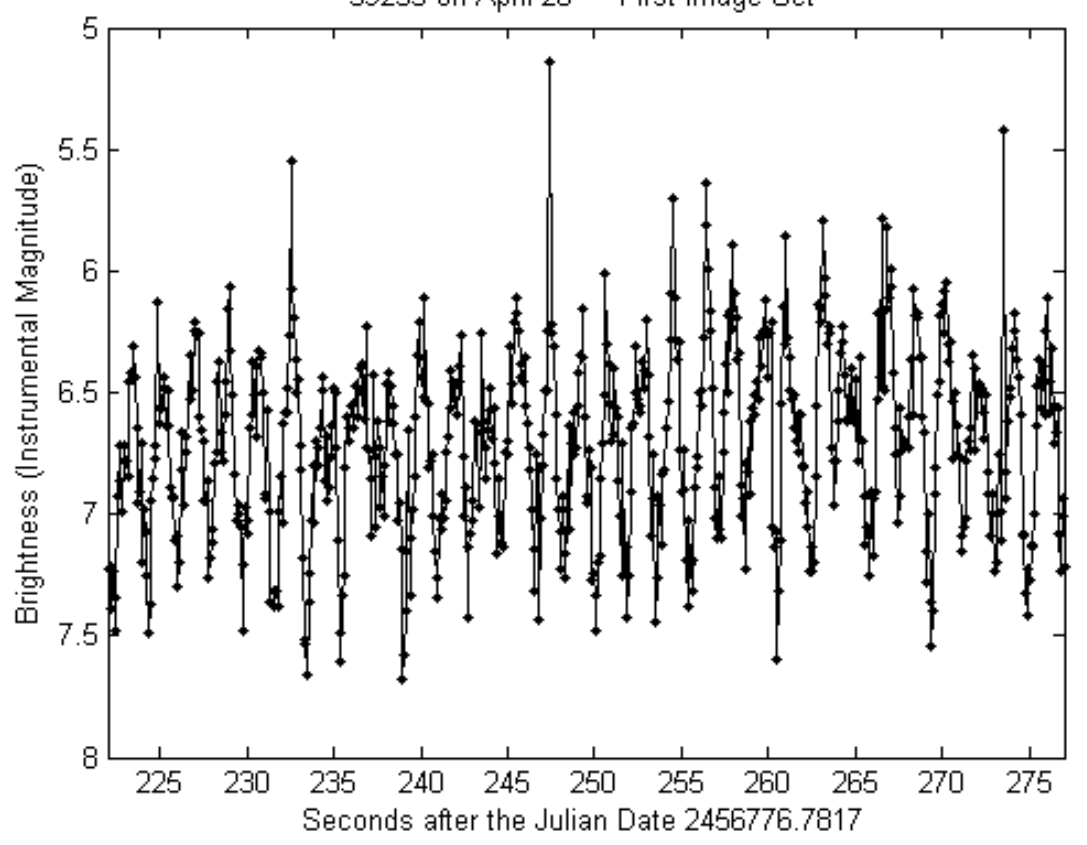


Light Curve

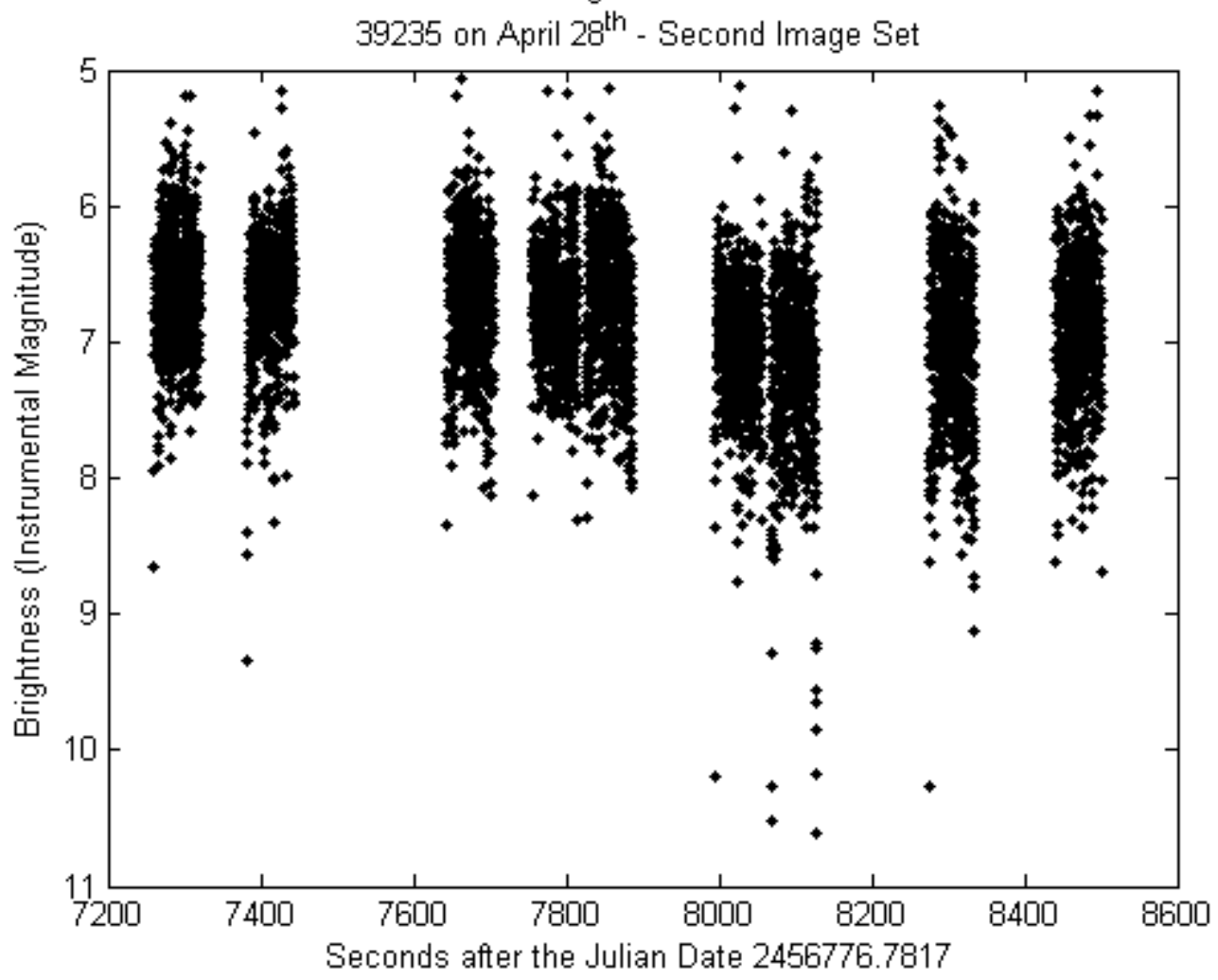

Portion of a Light Curve from one Image 39235 on April $28^{\text {th }}$ - Second Image Set

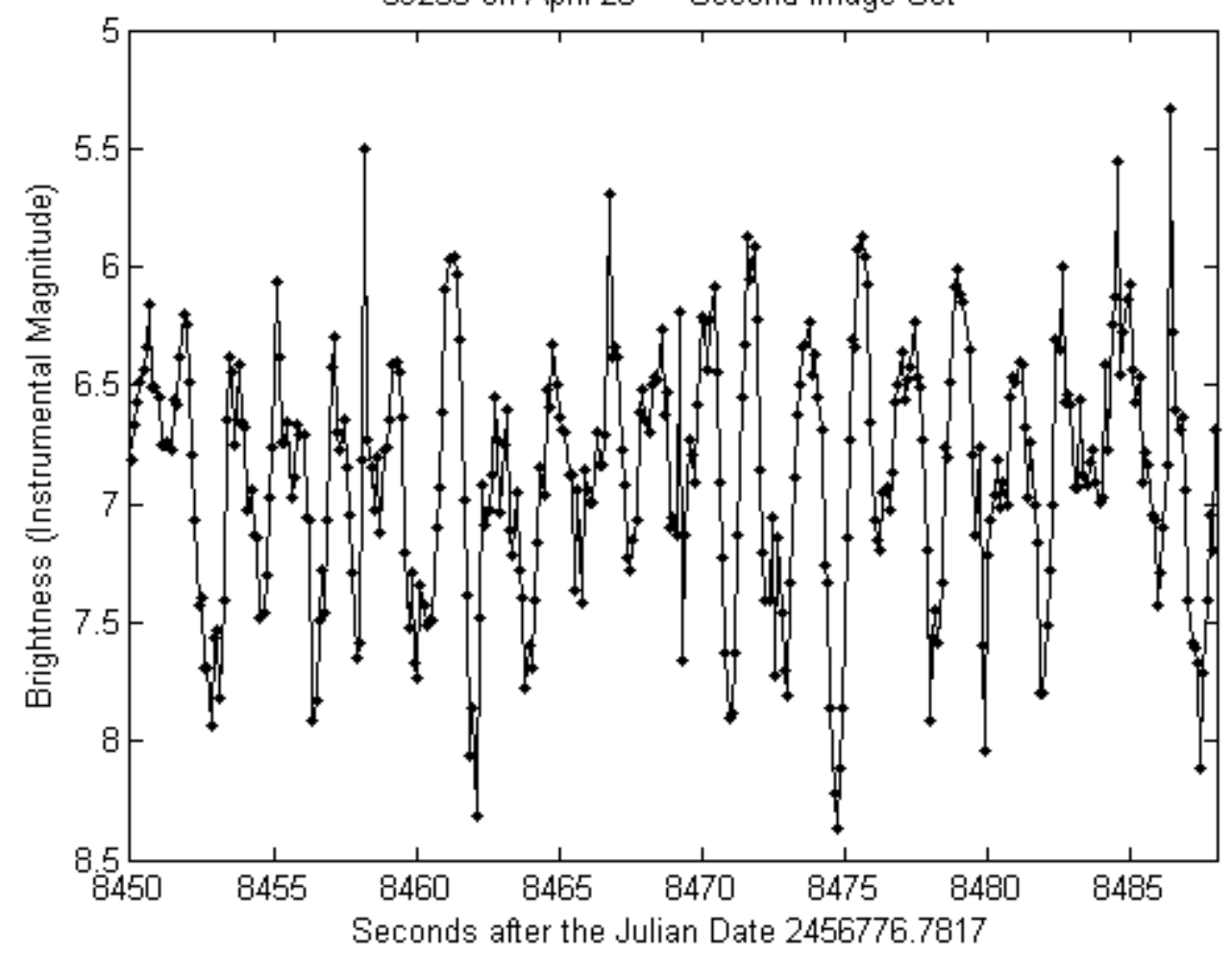




\section{APPENDIX F: Contour Plot Results for Observational Data}

Presented here are the contour plot results for the observational data. The sets of data were reduced night by night, and then as a whole, therefore, the contour plots are shown for each night, followed by the combination contour plot. The contour plots are made for the RA, DEC, and sidereal period combination that has the lowest overall score, and this sidereal period may not perfectly match the sidereal period presented in Table 4. The natural $\log$ of the scores is shown in the scale to the right of each image to properly display all contour levels. For many of the plots, all scores higher than a threshold value were "cut off" and are simply displayed as dark red. The scores are not comparable from image to image because the score depends on how many flashes were analyzed to create each individual plot. The number of flashes then varies with the number of images taken for each night of observation, but is not directly proportional.

\section{Target 36034}

April $28^{\text {th }}$

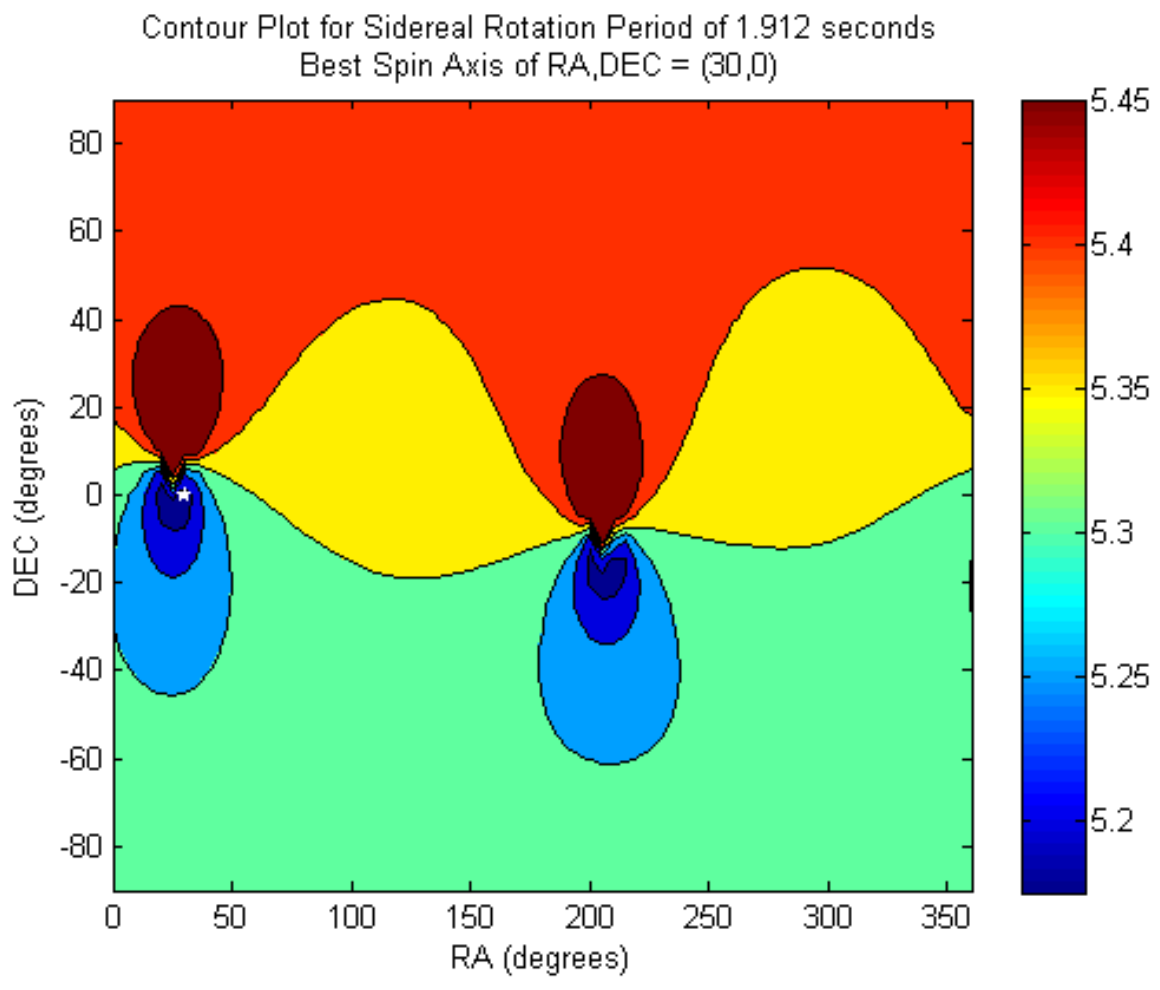


April $30^{\text {th }}$

Contour Plot for Sidereal Rotation Period of 1.918 seconds Best Spin Axis of RA,DEC $=(220,-5)$

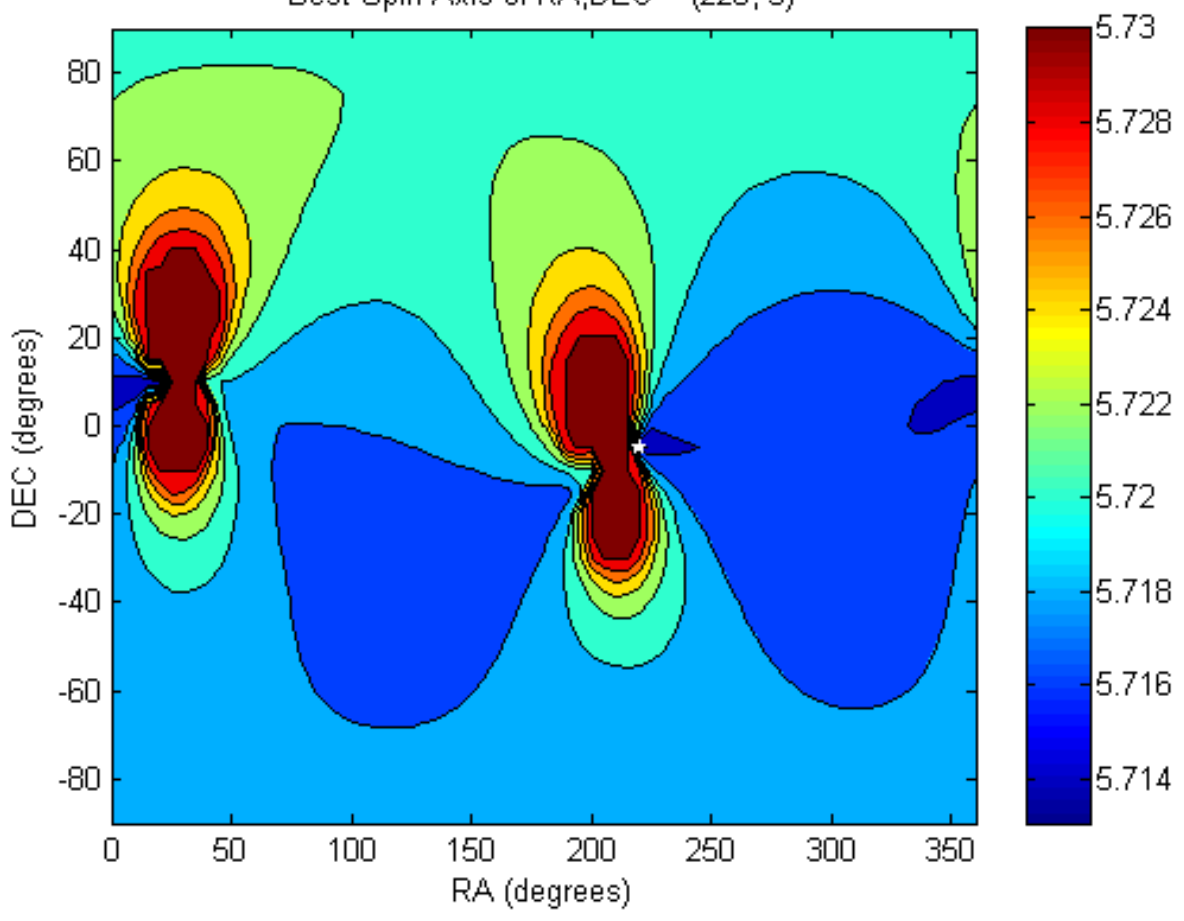

Combined Nights

Contour Plot for Sidereal Rotation Period of 1.91 seconds Best Spin Axis of RA,DEC $=(45,5)$

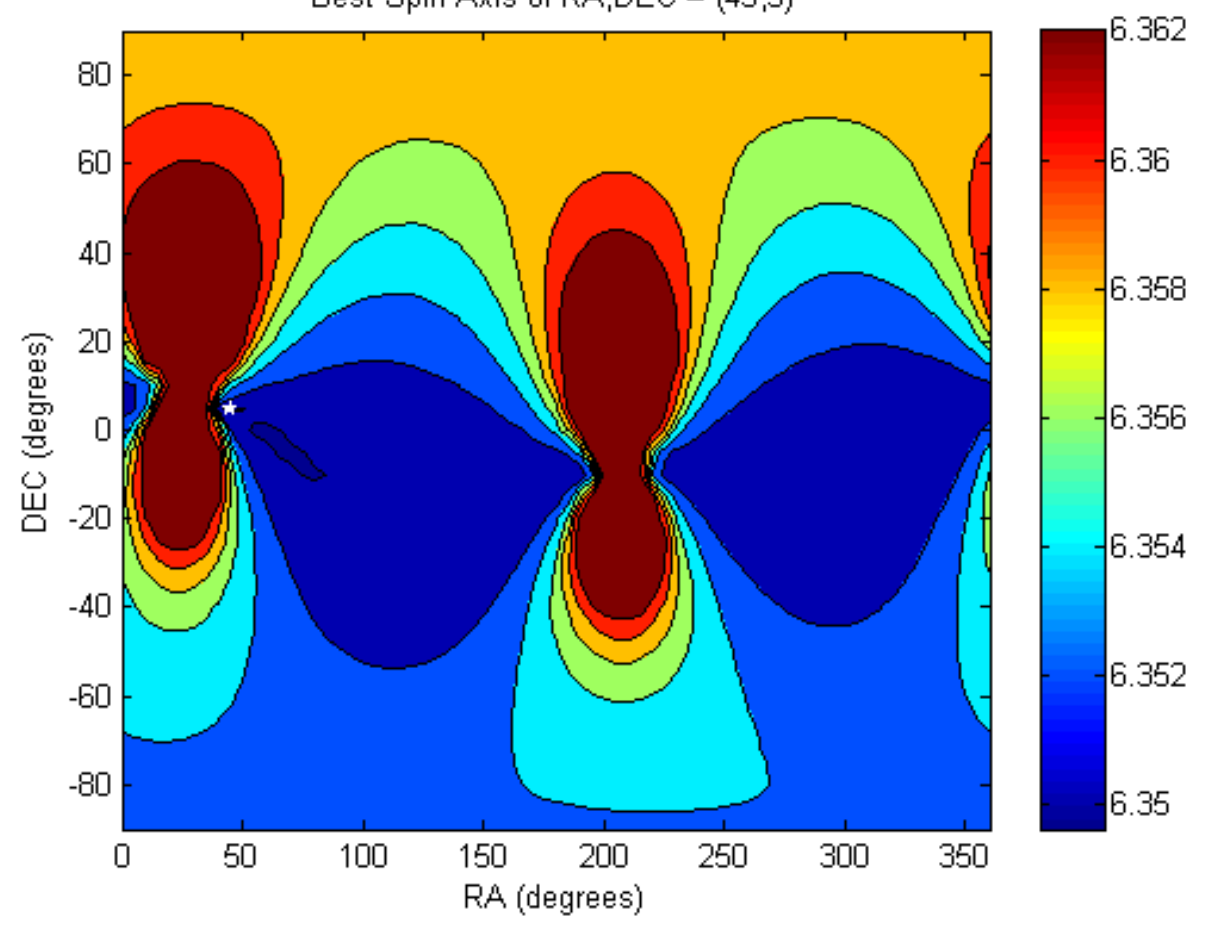




\section{2. $\quad$ Target 37777}

April $26^{\text {th }}$

Contour Plot for Sidereal Rotation Period of 3.647 seconds

Best Spin Axis of RA,DEC $=(215,-10)$

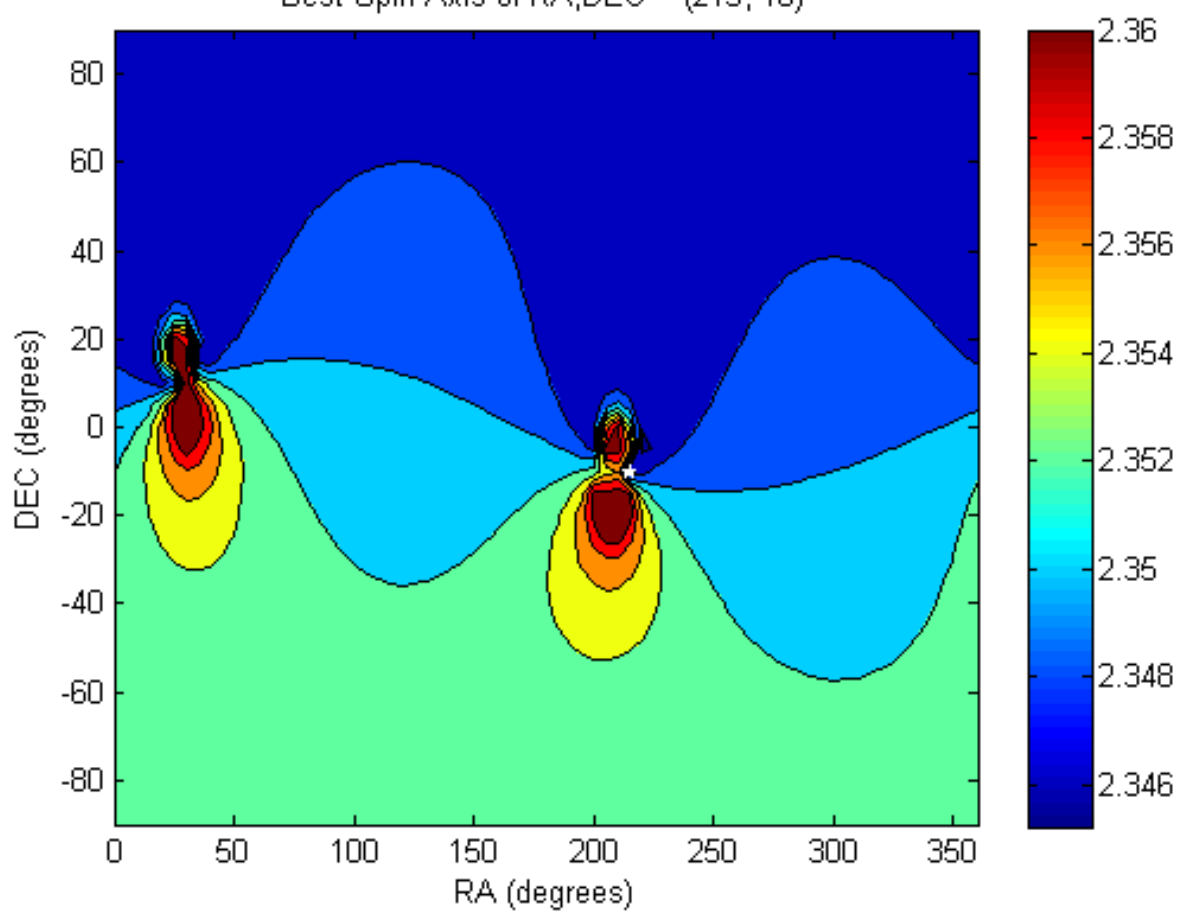

April $29^{\text {th }}$

Contour Plot for Sidereal Rotation Period of 3.647 seconds Best Spin Axis of RA,DEC $=(190,-10)$

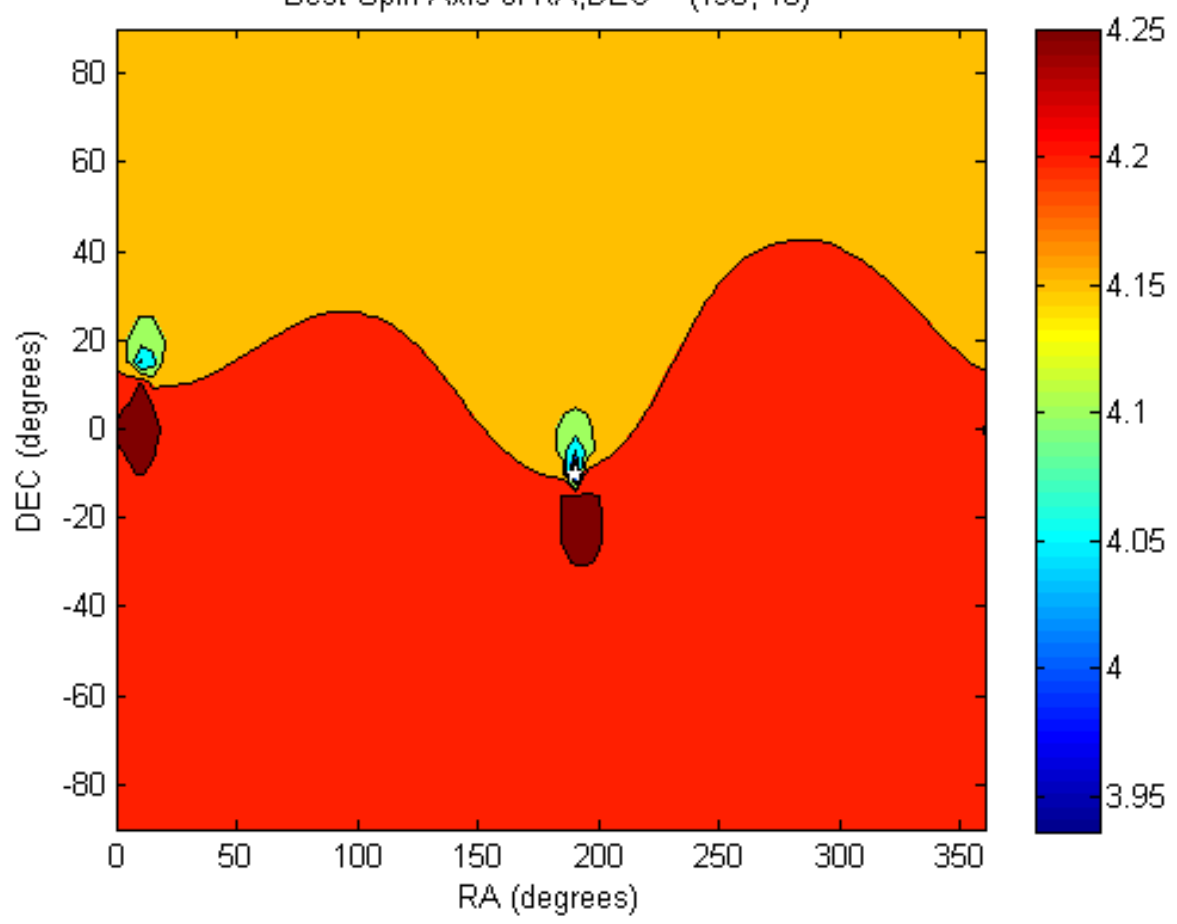


Combined Nights

Contour Plot for Sidereal Rotation Period of 3.647 seconds Best Spin Axis of RA,DEC $=(30,15)$

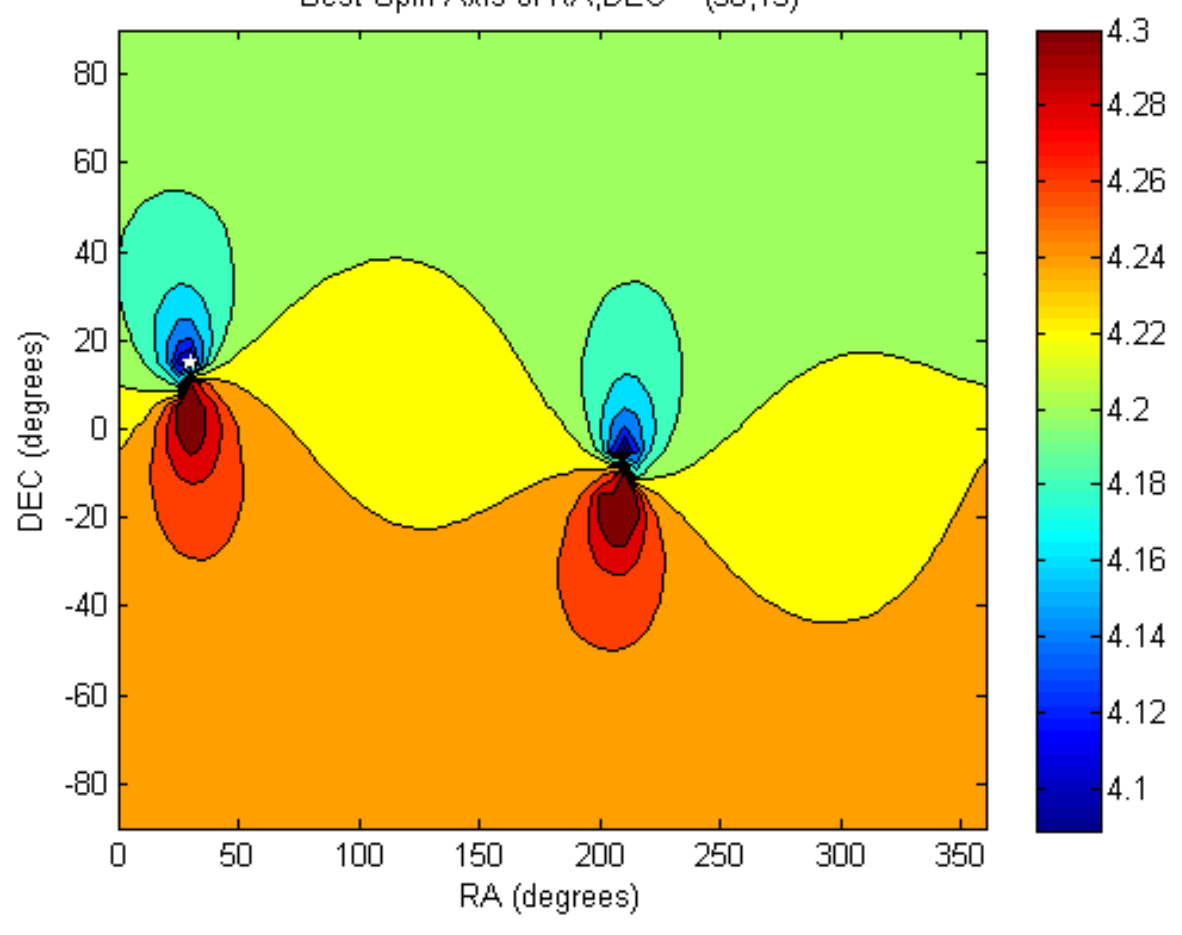




\section{Target 37811}

April $23^{\text {rd }}$

Contour Plot for Sidereal Rotation Period of 5.365 seconds

Best Spin Axis of RA,DEC $=(195,-5)$

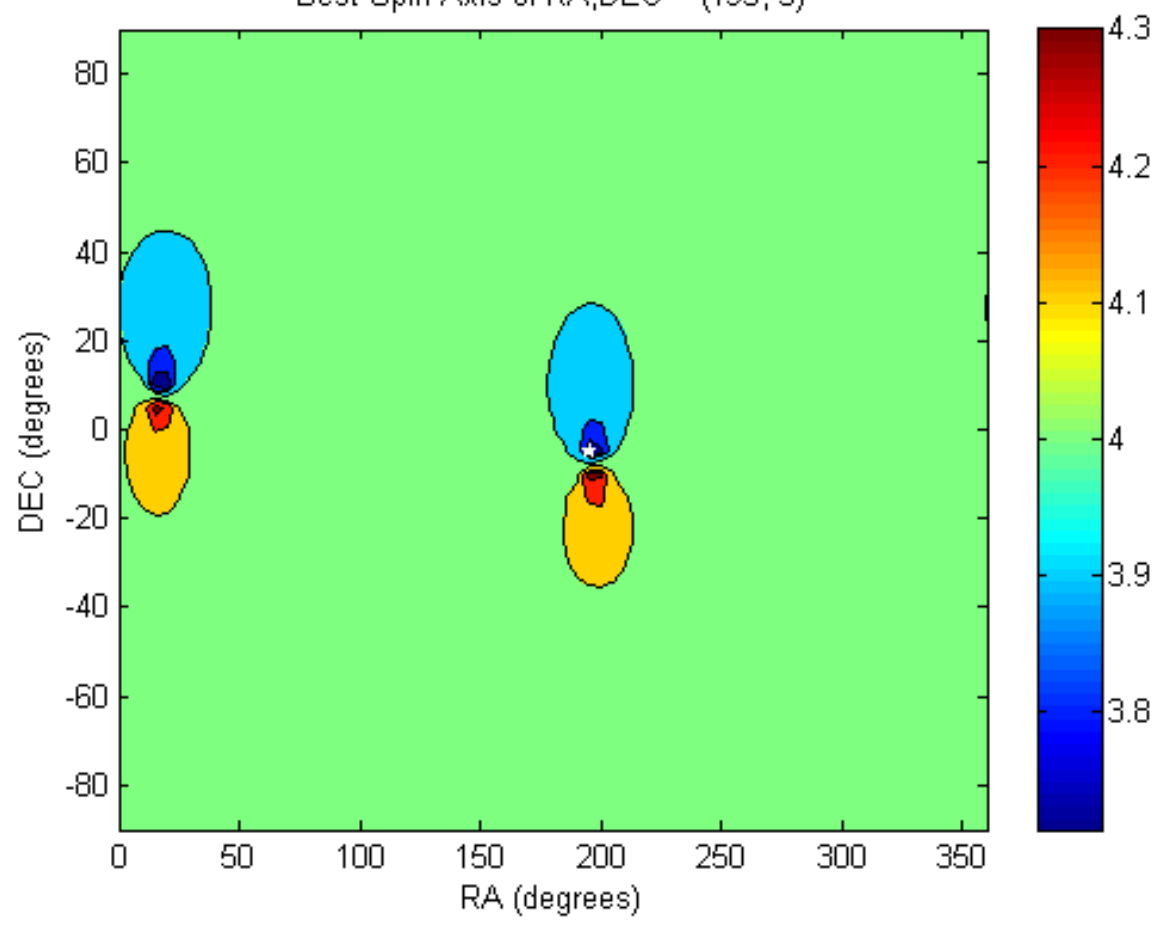

April $28^{\text {th }}$

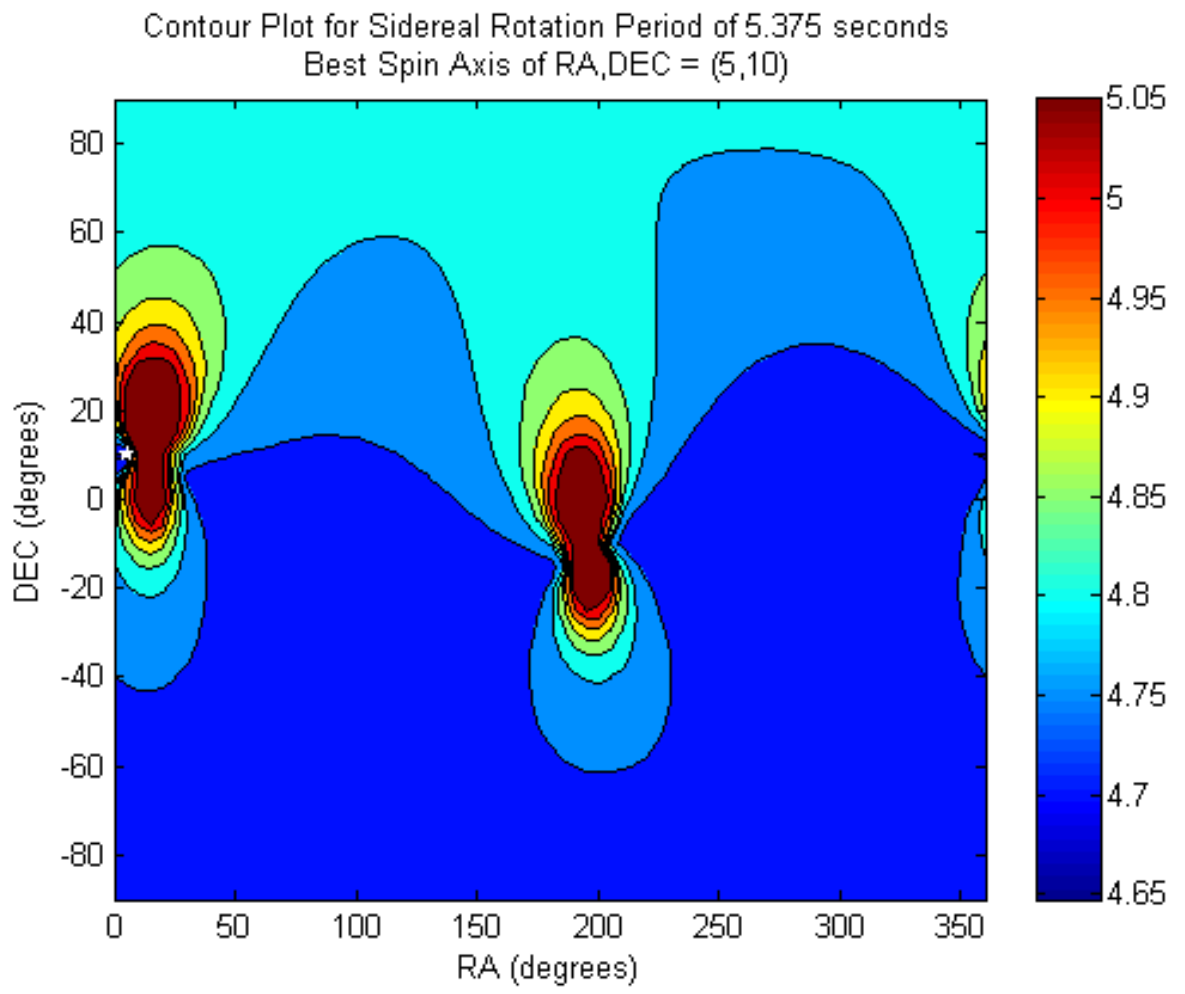


May $1^{\text {st }}$

Contour Plot for Sidereal Rotation Period of 5.38 seconds

Best Spin Axis of RA,DEC $=(210,-10)$

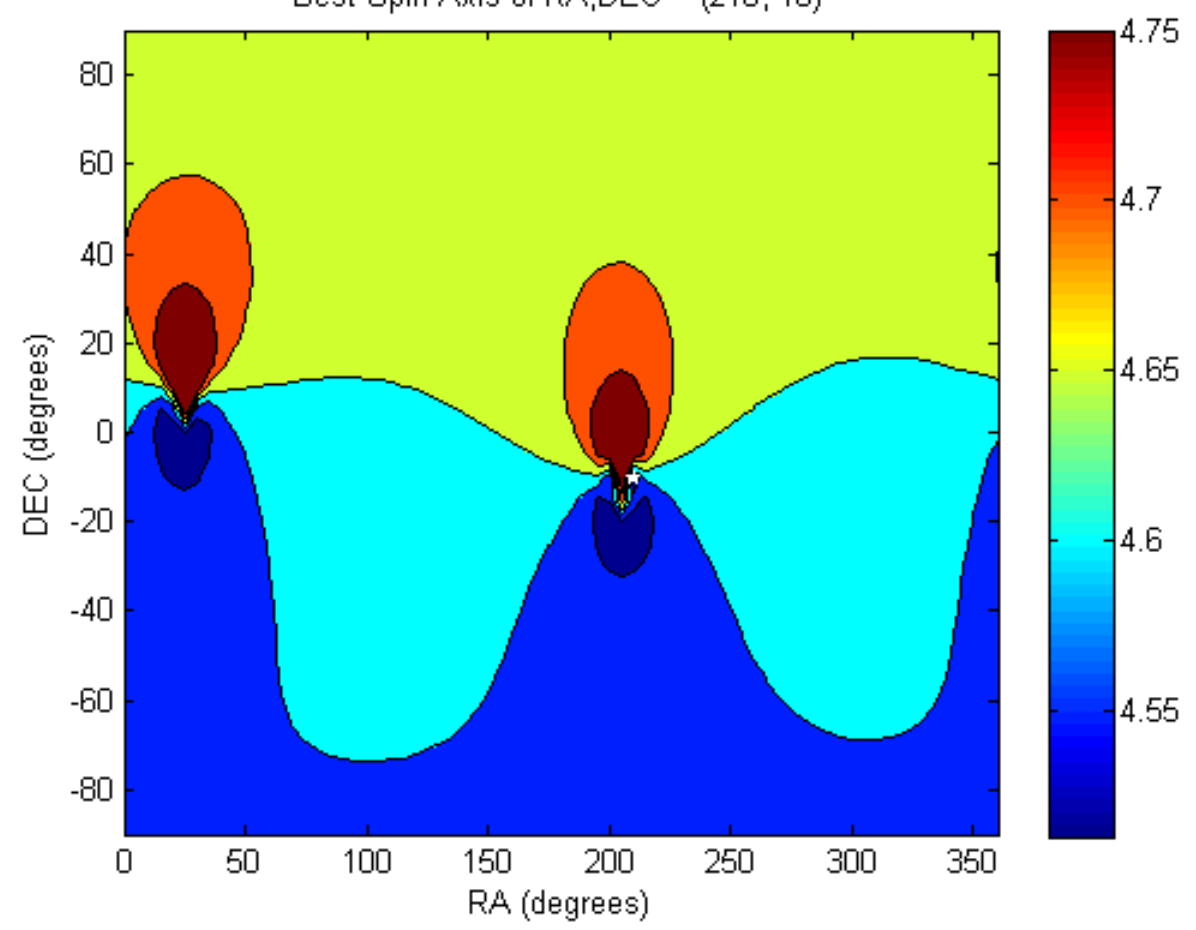

Combined Nights

Contour Plot for Sidereal Rotation Period of 5.375 seconds Best Spin Axis of RA,DEC $=(0,5)$

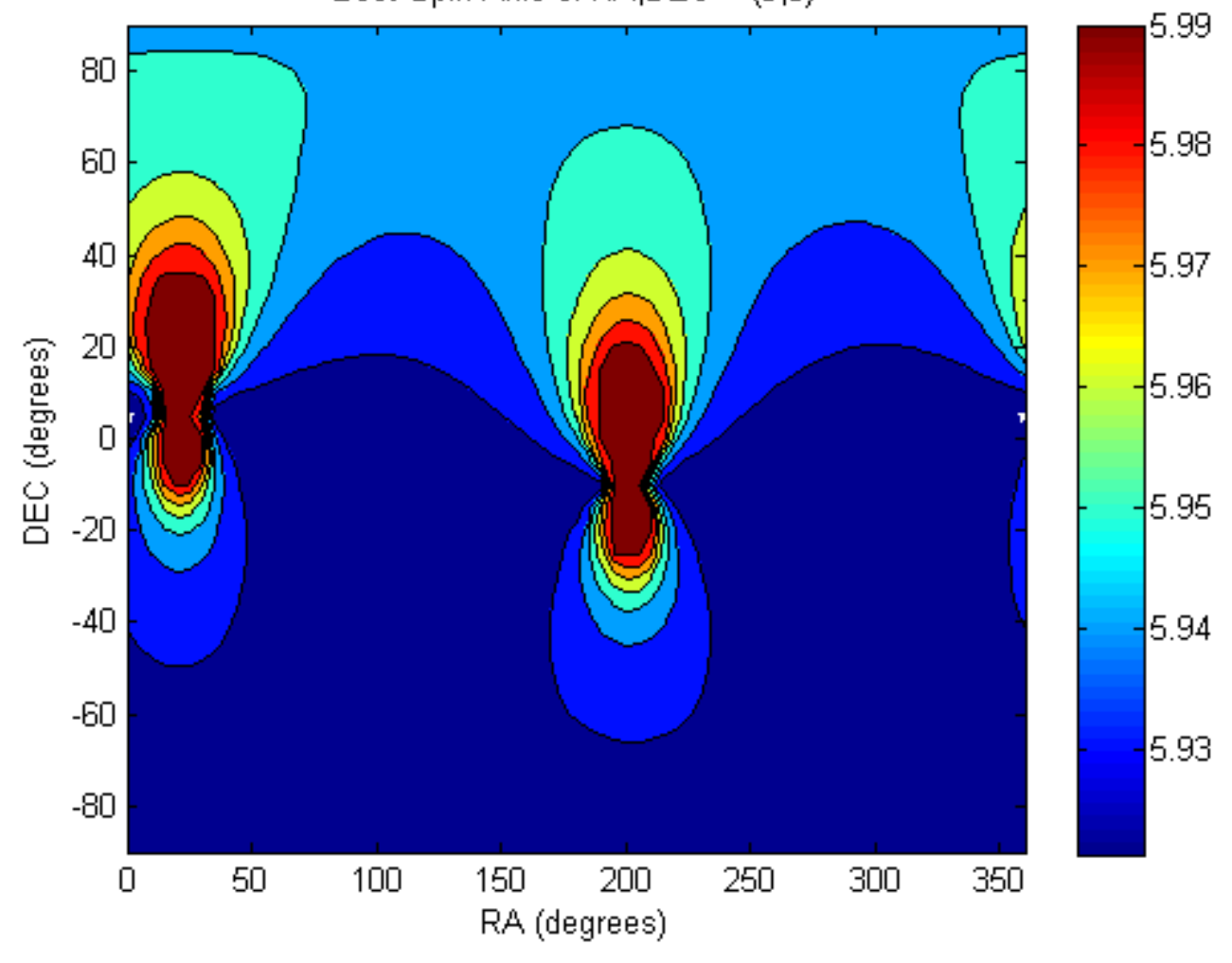




\section{Target 39218}

April $23^{\text {rd }}$

Contour Plot for Sidereal Rotation Period of 11.205 seconds

Best Spin Axis of RA,DEC $=(185,-10)$

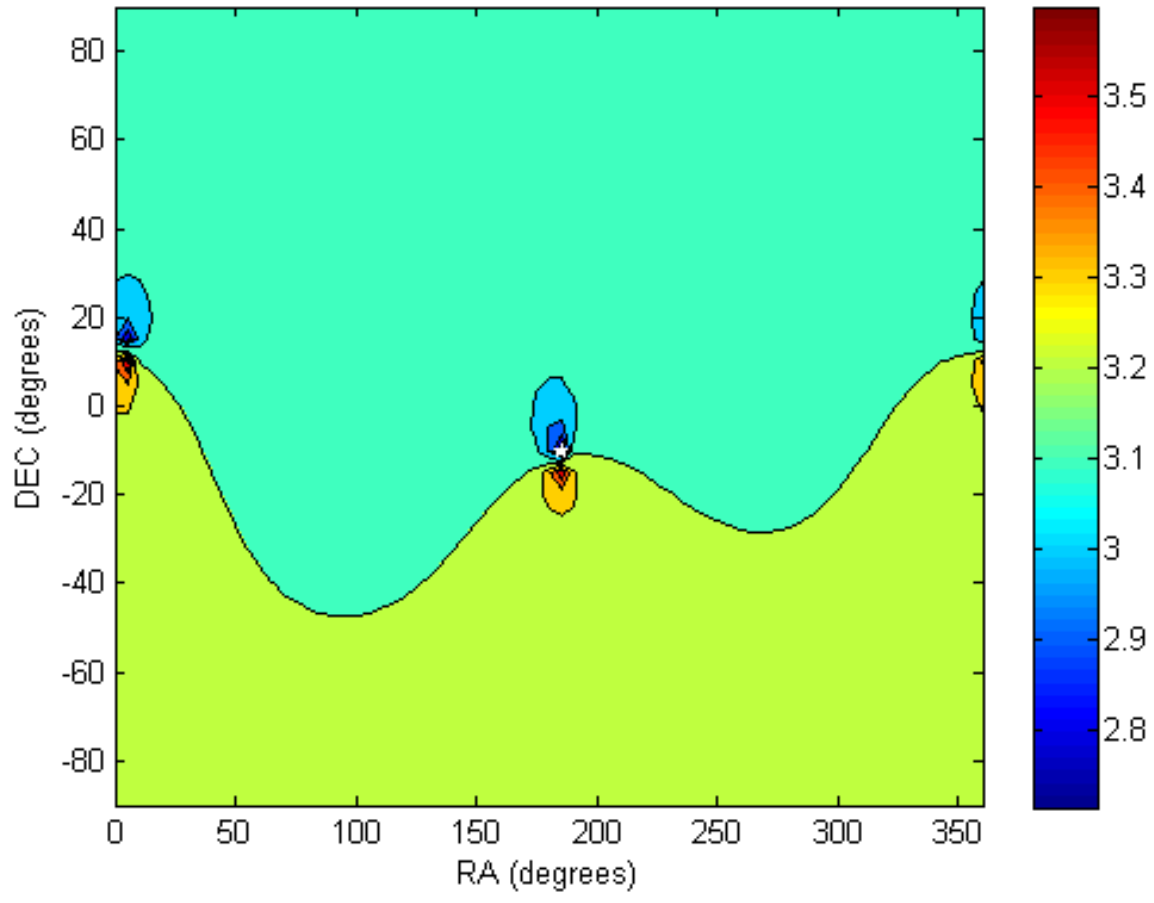

April $26^{\text {th }}$

Contour Plot for Sidereal Rotation Period of 11.23 seconds Best Spin Axis of RA,DEC = $(30,5)$

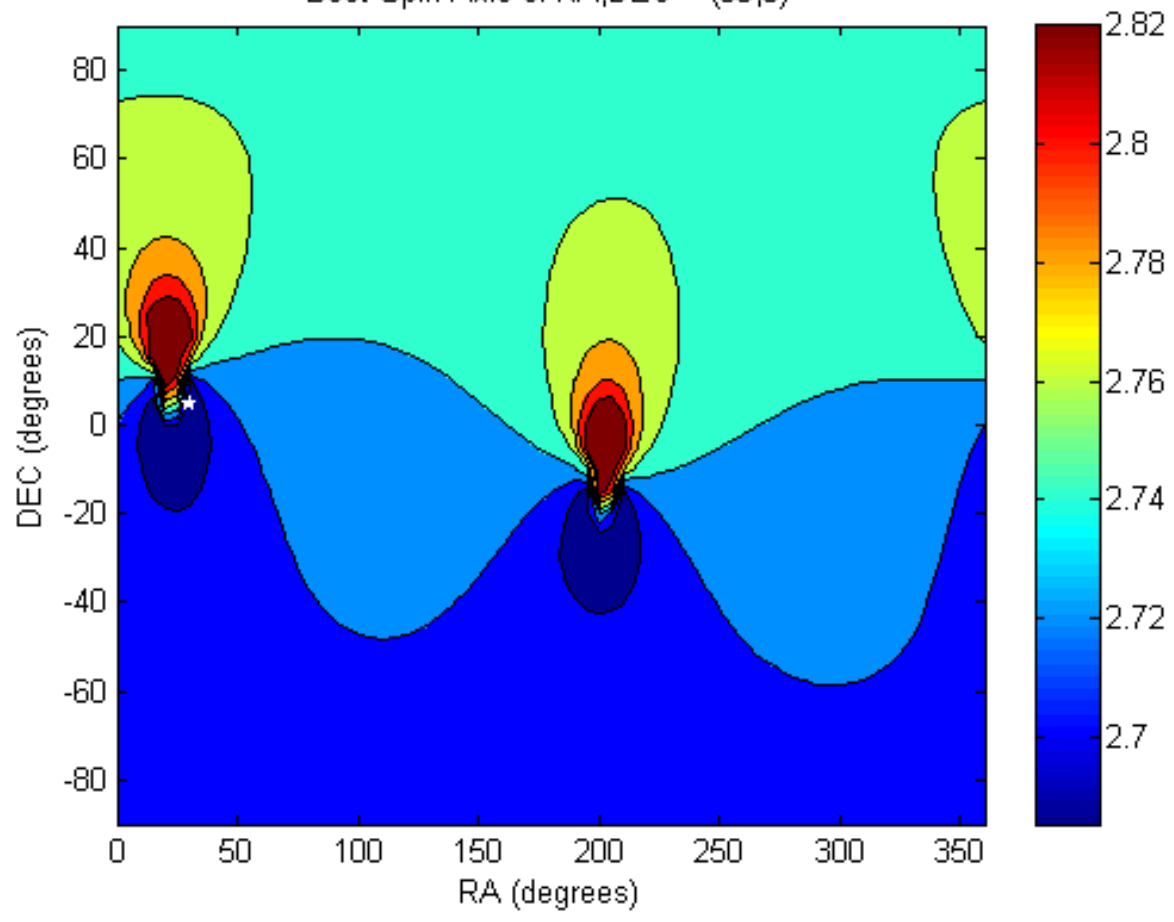


April $29^{\text {th }}$

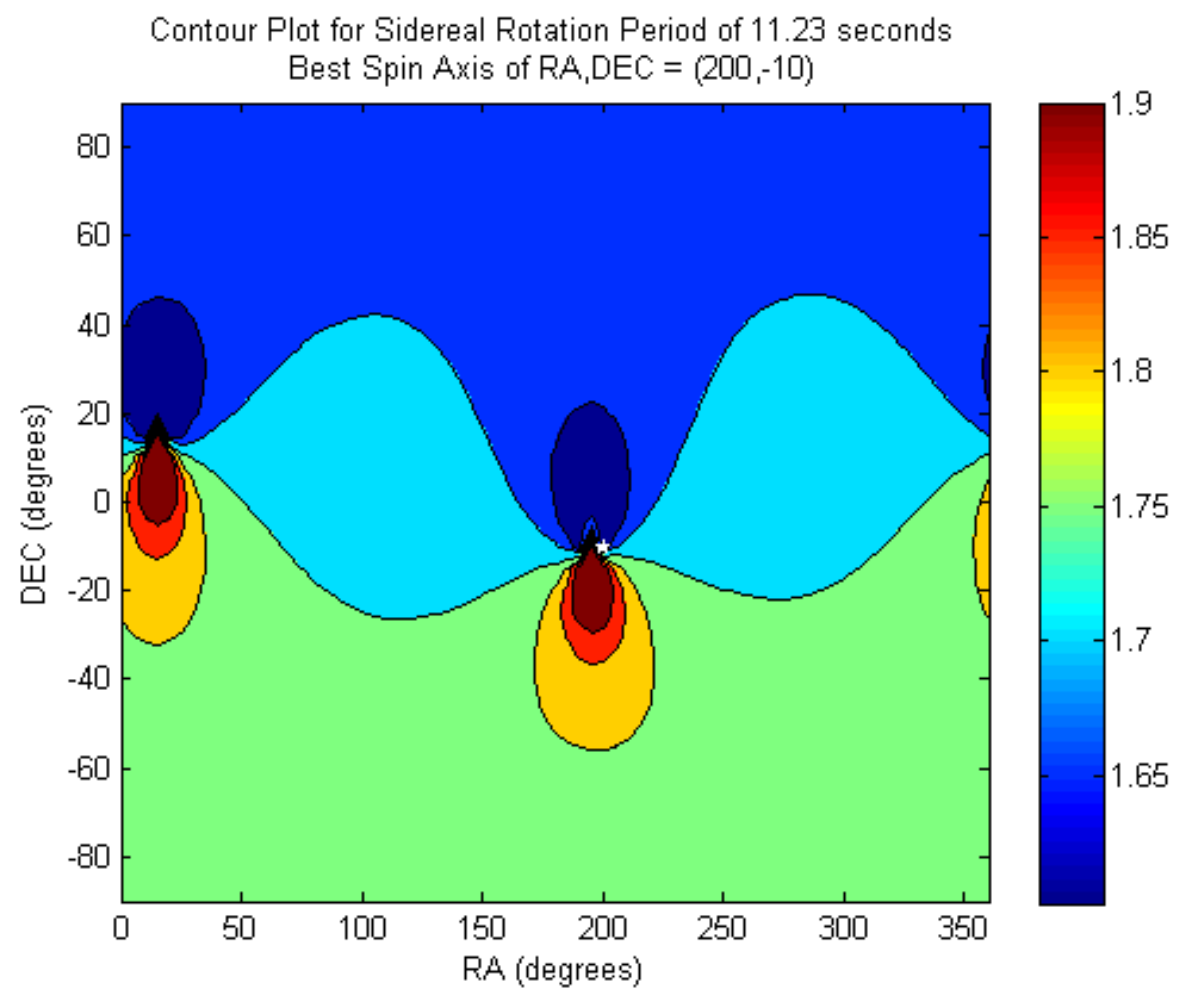

Combined Nights

Contour Plot for Sidereal Rotation Period of 11.205 seconds Best Spin Axis of RA,DEC $=(185,-10)$

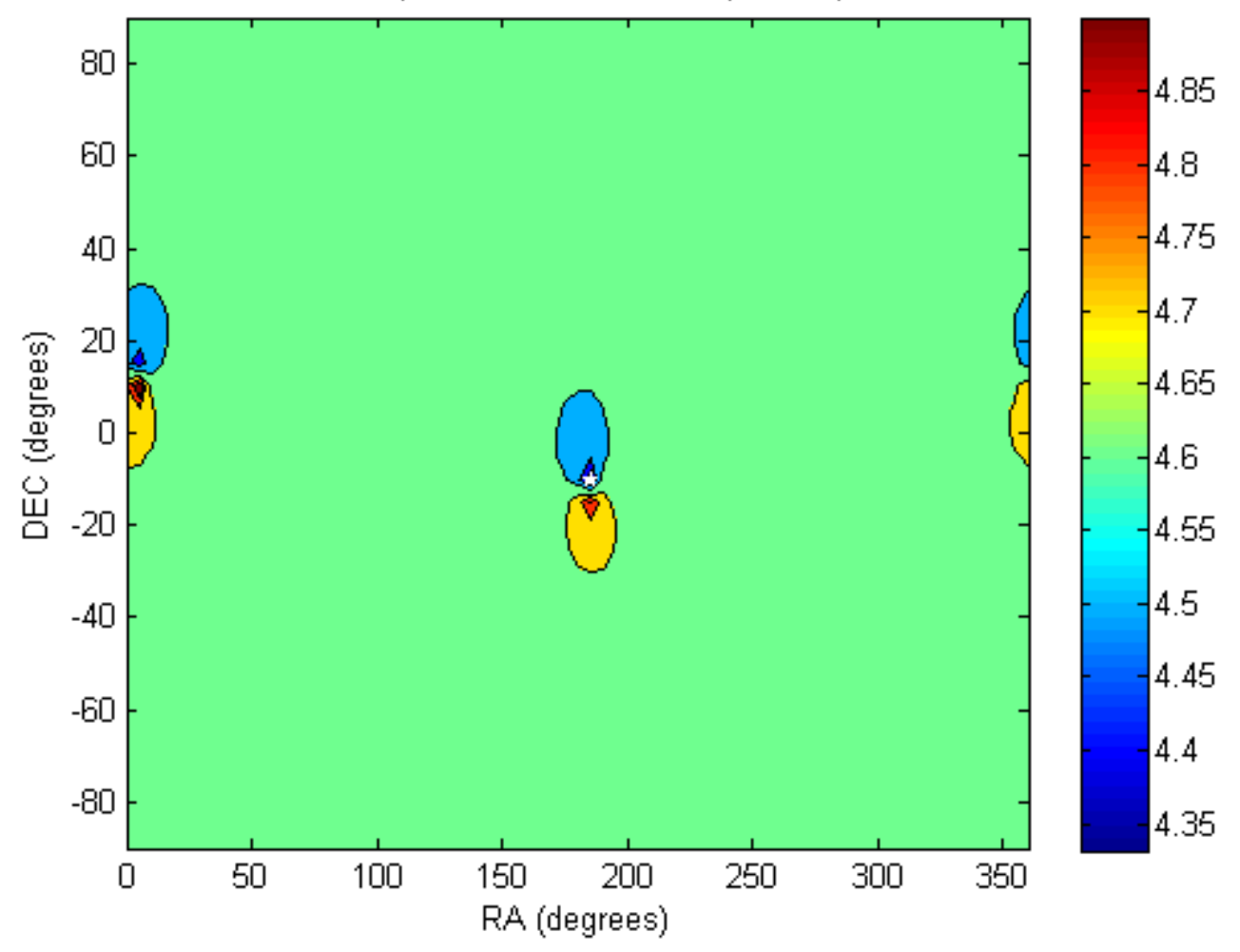




\section{Target 39235}

April $28^{\text {th }}$

Contour Plot for Sidereal Rotation Period of 3.6005 seconds

Best Spin Axis of RA,DEC $=(200,-10)$

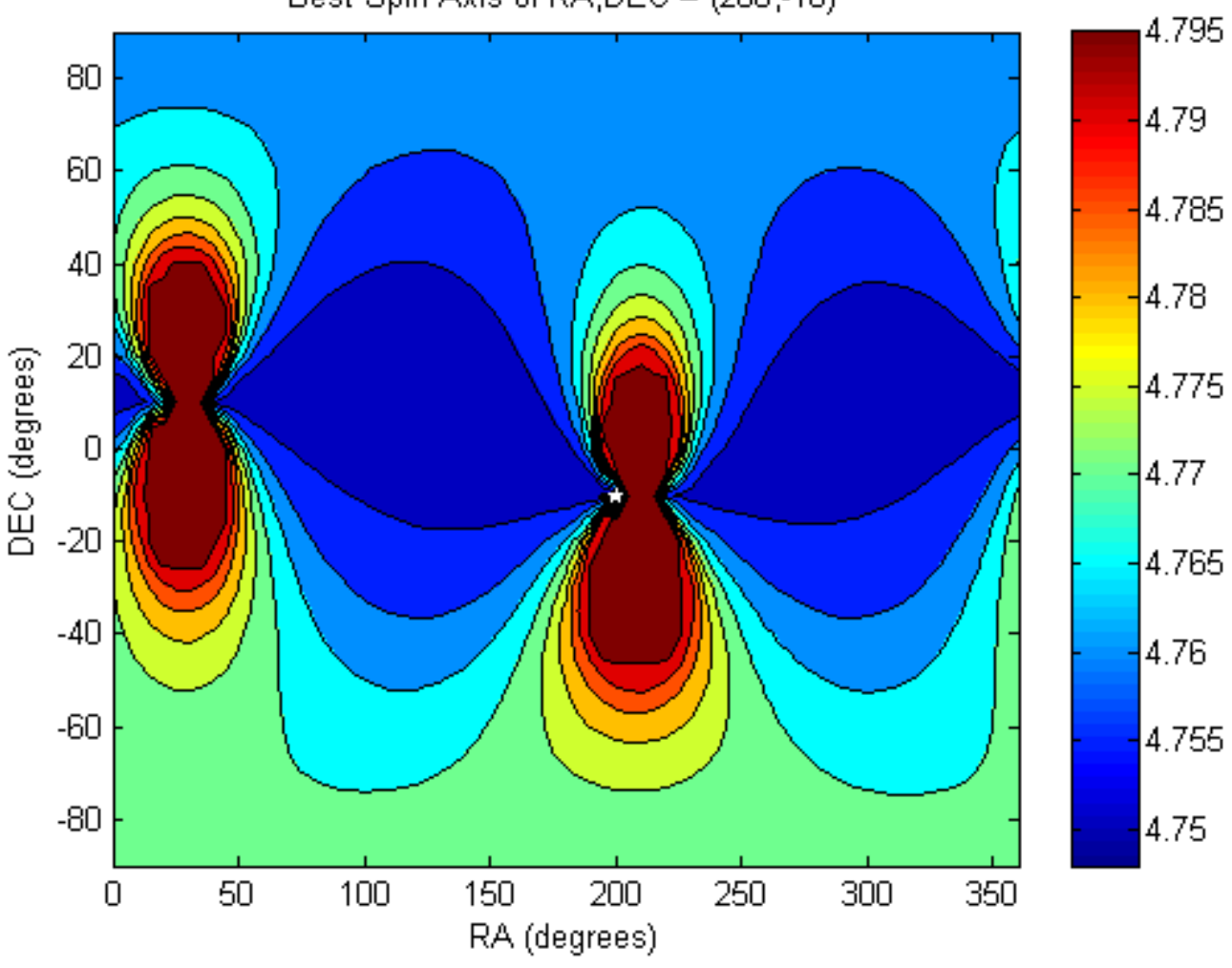

